IBI -31926

DE92 017122

\title{
The Dynamics of Azulene in Liquids and Compressed Gases on Ultrafast Timescales
}

\author{
Karen E. Schultz \\ Ph.D. Thesis \\ Department of Chemistry \\ University of California \\ and \\ Chemical Sciences Division \\ Lawrence Berkeley Laboratory \\ University of California \\ Berkeley, CA 94720
}

February 1992

This work was supported in part by the National Science Foundation, by the Office of Naval Research, and by the Director, Office of Energy Research, Office of Basic Energy Sciences, Chemical Sciences Division, of the U.S. Department of Energy under Contract No. DE-AC03-76SF00098. 
The Dynamics of Azulene in Liquids and Compressed jases on

$$
\begin{gathered}
\text { Ultrafast Time Scales } \\
\text { by } \\
\text { Karen Elizabeth Schultz } \\
\text { Abstract }
\end{gathered}
$$

The ultrafast dynamics of vibrationally hot ground state azulene molecules have been time resolved by picosecond transient absorption spectroscopy in a variety of solvents including hexane, chloromethanes, methanol, $\mathrm{CClF}_{3}, \mathrm{Xe}$ and $\mathrm{Kr}$. A high pressure optical cell was used to liquify gases for use as solvents and change their density and temperature, independently, over the entire liquid density range. Experimental results indicate that the vibrational cooling rate is strongly solvent dependent, with cooling rates of approximately $20 \mathrm{psec}$ in molecular solvents and approximately 150 psec in atomic solvents. comparison of the rates in $x e$ and $\mathrm{Kr}$ at constant density demonstrates the strong effect of solvent mass on energy transfer. The effect of solvent temperature on vibrational coolirg is minimal, as is the effect of solvent density. This latter result is quite surprising in light of earlier experiments on simpler molecular systems, such as $I_{2}$ in $X e$. This anomalous density effect is examined in light of Isolated Binary Collision (IBC) theory and bulk thermal transport models. Both theories accurately model all experimental results obtained with the 
exception of the density effect. Possible explanations for the breakdown of IBC theory in this case are offered along with methods to improve IBC theory for application to complex three dimensional molecular systems. 
In memory of

my grandfather

Philip Mullenbach

$(1913-1989)$

i. i 


\section{Table of contents}

INTRODUCTION

ACKNOWLEDGEMENTS

Chapter 1: BACKGROUND .

1.1 Azulene spectroscopy . . . . . . . . . . . . 1

1.1.a General spectroscopy . . . . . . . . . . . . 1

1.1.b Vibrational cooling spectroscopy ........ 3

1.1.6 Heavy Atom Effect.................. 4

1.1.d Pressure Dependent Absorption Shifts . . . . . 6

1.1.e Azulene Absorption Spectrum . . . . . . . . 8

1.1.f Mode Analysis of Azulene . . . . . . . . . 8

1.2 Experimental Studies of Energy Transfer in Azulene . - 14

1.2.a Gas Phase Studies of Energy Transfer in Azulene 15

1.2.b Theoretical Studies of Energy Transfer in Azulene ................ 19

1.2.c Crystal studies of Energy Transfer in Azulene . 20

1.2.d Matrix Studies of Energy Transfer in Azulene . 21

1.2.e Liquid Study of Energy Transfer in Azulene . . 22

References for chapter 1 .............. 24

Chapter 2: EXPERIMENTAL . . . . . . . . . . . 27

2.1 Picosecond Laser System . . . . . . . . . . 27

2.2 Polarization control Experiments . . . . . . . 30

2.3 High Pressure System................ 33

2.4 Sample preparation .............. 35 
2.5 Excited State Dynamics Studies . . . . . . . . . .

2.6 Solvent Thermodynamic Information . . . . . . . . . . 40

References for chapter 2 . . . . . . . . . . . . . . 45

Chapter 3: RESULTS . . . . . . . . . . . . . . . . 47

3.1 General Information . . . . . . . . . . . . . . 47

3.1.a Data Fitting process . . . . . . . . . . . 47

3.1.b Stimulated Emission Detection . . . . . . . 50

3.2 Solvent Trends . . . . . . . . . . . . . . . . 53

3.2.a Probe Wavelength Dependence . . . . . . . . 53

3.2.b Solvent Mass Dependence . . . . . . . . . . 56

3.2.c Temperature Dependence . . . . . . . . . . 59

3.2.d Solvent Density Dependence . . . . . . . . . 59

3.2.e Solvent Isotope Effect . . . . . . . . . . . 67

3.2.f Solvent Dependence . . . . . . . . . . . 68

References for chapter 3 . . . . . . . . . . . . . . . 71

Chapter 4: ANALYSIS . . . . . . . . . . . . . . . 73

4.1 Vibrational Relaxation studies of $I_{2}$ in Compressed Gases . . . . . . . . . . . . . . . . . 73

4.2 Isolated Binary Collision Theories . . . . . . . . . 76

4.3 Molecular Dynamics Simulations of Solvent Effects . . 86

4.4 Bulk Thermal Diffusion Theories . . . . . . . . . . . 89

4.5 Liguid Phase Vibrational Relaxation Studies of Azulene 110 
References for Chapter 4 . . . . . . . . . . . . . 115

Chapter 5: SUMMARY AND CONCLUSIONS . • • • . • . • . . • • 117 APPENDIX . . . . . . . . . . . . . . . . . . . . . 122 


\section{INTRODUCTION}

Vibrational relaxation plays a central role in condensed phase dynamics. The time scale on which a vibrationally hot molecule loses its energy to the solvent is critical to the overall molecular kinetics. Previous studies have investigated simple molecular systems to investigate the process of energy flow within and among molecules. Some of these studies examined diatomic molecules in liquids and gases in an attempt to simplify the system and illuminate the basic chemical dynamics. However the role of the solvent in this process is not yet fully understood, which limits application to and understanding of more complicated and more common chemical systems. The goal of this work is to extend current understanding of energy transfer to complex molecules in solution.

Collisions between molecules in solution are an important means of energy transfer in the liquid phase. These collisions happen rapidly, on the order of hundreds of femtoseconds. As a result, study of liquid phase dynamics must be able to catch rapid transient phenomena on this time scale. Additionally, collisions are affected by many factors - temperature speeds the collision rate, pressure changes the density of molecules in solution and the packing geometry of the solvent molecules, different solvents pack together in different structures despite minor chernical changes. 
Experimental control of these factors is important for meaningful and understandable results.

Three components are used experimentaliy to provide control of these factors. A high pressure optical cell can be used to control the thermodynamics of the solution, regulating the solvent temperature and density over a range of values. Picosecond laser spectroscopy provides the energy and wavelength range needed to accurately examine the rapid dynamics in a condensed media. A spectroscopically well understood solute molecule facilitates the analysis of reaction dynamics by reducing the number of different processes in the system of study. These three methods are used in this work to illuminate vibration cooling cf moderately sized molecules.

In order to gain insight into the dynamics of larger molecular systems, an investigation into the cooling of ground state azulene was begun. Azulene is an ideal candidate for studying the process of vibrational relaxation in a complex molecular system. Its spectroscopy has been well studied, forming a foundation on which this research can build. It readily absorbs light in the visible region of the spectrum, the wavelengths where the most intense ultrafast pulses can easily be made. There are few excited states hich complicate the spectroscopy or interfere with the dynamics. Additionally, photoisomerization to naphthalere is not a problem. Because of the rapid internal conversion from the 
first excited state, a hot population of ground state azulene can be produced for investigation. Fortunately, azulene dissolves in many solvents including liquified zenon and kxypton (though none of the other noble gases), providing a wealth of data for comparison.

The azulene/rare gas system provides a unique opportunit: for clarifying liquid phase dynamics. Compressed noble gases are the simplest solvent systems available. They lack all internal degrees of freedom, which simplifies energy flow from solute to solvent. They are chemically inert and will not strongly interact with the solute. With temperature and pressure controlled independently, one can alter the solvent environment without changing the solvent itself. This allows accurate study of the effect of solvent environment on liquid phase energy transfer.

The role of the solvent environment (temperature, density and molecular complexity) on the vibrational relaxation rate is experimentally determined by altering the thermodynamic state of the noble gas solvent and examining the consequent change in the relaxation rate of azulene. The effect of the solvent on vibrational cooling is monitored by transient absorption spectroscopy of the ground state population. These results must then be examined in light of previous studies of condensed phase energy transfer. The relaxation dynamics of diatomics such as iodine have been comprehensively studied, by both experiment and theory. The results of these studies can 
be compared and contrasted to test their application to more complicated molecules such as azulene. A comparison between the vibrational relaxation of a diatomic and a polyatomic, under identical solvent conditions, provides insight into the different dynamics of complex solute molecules.

The results have been modelled with a number of different theories. Bulk energy transport theories examine the effectiveness of describing a liquid phase system by a single experimental parameter (the thermal conductivity) and correlating this parameter with the vibrational cooling rate. Alternatively, gas phase theories such as Isolated Binary Collision theory relate the microscopic behavior of colliding molecules in solution to the vibrational relaxation dynamics. Molecular dynamics simulations provide a theoretical picture of the dynamics in solution on an atomic scale, given the interactions between particles. These models are used to decipher the effect that solvent has on the vibrational cooling dynamics of azulene. 


\section{ACKNOWLEDGEMENTS}

First and foremost, I am thankful for the love and support of my parents, without whom I would not be. My mother has been my rock of Gibralter, seeing me through the ups and downs of life. My father remains my number one fan, eternally confident in my ability to do anything. My uncle Guy has been my surrogate parent during my five and half years here in Berkeley. He has made graduate school a special experience for me.

I appreciate the independence that my advisor, Charles Harris, gave me to pursue the azulene project. It prored to me that I had the wherewithal to be a good scientist. I have particularly enjoyed the camraderie of the folks who have made up the Harris group during my graduate career. They have been like a family to me, especially my officemates who have endured more than their share of abuse. If it weren't for them, I would never have learned about surface science. Some group members have been especially important to this research and I have listed them and their contributions.

Mark Paige introduced me to the laser system and gave me Alf, his homemade high pressure cell. With the patience of Job, he answered my continual questions. Most importantly, he was the first person to have confidence in the azulene project and without his faith, I might never have pursued it. walt Merry has been my insufferable coworker for these many years 
(or have I been his?); always there with a hand to lend, a shoulder to cry on and an ear to listen. He was especially supportive during my first horrible year here. Dr. Dan Russell carried out the three dimensional molecular dynamics simulations on azulene and has been great to work with. He is not only a good scientist but a true gourmet. our conversations have taught me as much about science as food. Keenan Brown's musings, hypotheses, suggestions and ideas about science have been continually helpful and educational to me. Linda Marquez of the stacy group at UC Berkeley helped with the analysis using bulk thermal cooling models. Last but not least, there is Jason king, the heir to picosecond jockdom: a good friend, a gifted scientist and thankfully, a staunch Democrat!

I. would also like to acknowledge (chronologically) women who have made an impact on my scientific career. Jill Bonham costa was my first lab partner, assisting me with experiments using crayons and shampoo in my Kenner Easy Bake Oven. Kathy Brown-wensley has been my mentor for almost ten years. stephanie schaertel and I helped each other through chemistry at Swarthmore and beyond. Alex Weavex provided critical help and emotional support during the early, difficult years here at Berkeley. Finally, my dear friend of 16 years, Lydia Gregoret, has seen me through Mrs. Tier's Great Acid \& Base Test, as well as thesis writing and everything in between. I can't thank her enough for her continuing friendship. 
This work was supported by the National science Foundation and the office of Naval Research. The J. S. Department of Energy, office of Rasic Energy Sciences, Chemical sciences Division, under Contract No. DE-AC02765 Fo0098 is also acknowledged for some specialized equipment used in these experiments. 
Chapter 1: BACKGROUND

\subsection{Azulene spectroscopy}

In order to simplify discussion of the experimental results, an overview of the known spectroscopy of azulene is provided here. Although this research focuses on the ground state dynamics of azulene, the first and second excited states as well as low lying triplet states will be relevant to understanding this system. An energy level diagram of these states is shown in Figure 1.1 .

\section{1.a General Spectroscopy}

Azulene is particularly well known for its deviation from Kasha's Rule, which states that the lowest energy excited state of a molecule is strongly fluorescent." In azulene, the second excited state is more strongly fluorescent $\left(\phi_{2 \rightarrow 0}=\right.$ $0.031)^{2}$ than the first excited state $\left(\phi_{1 \rightarrow 0}=8 \times 10^{-6}\right) .^{3}$ This anomalous behavior was first discovered by Beer and LonguetHiggins in $1955^{4}$ and has caused many spectroscopists to investigate the photophysics of the azulene system. 5 Thus a foundation for understanding the dynamics of a polyatomic system was laid to further the present investigation of the dynamics of azulene.

The band origin for $S_{1}$ is approximately $14350 \mathrm{~cm}^{-1}$ while the band origin for $S_{2}$ is approximately $29000 \mathrm{~cm}^{-1}$. The $S_{0}-S_{1}$ transition has an absorption strength of about 300-400 


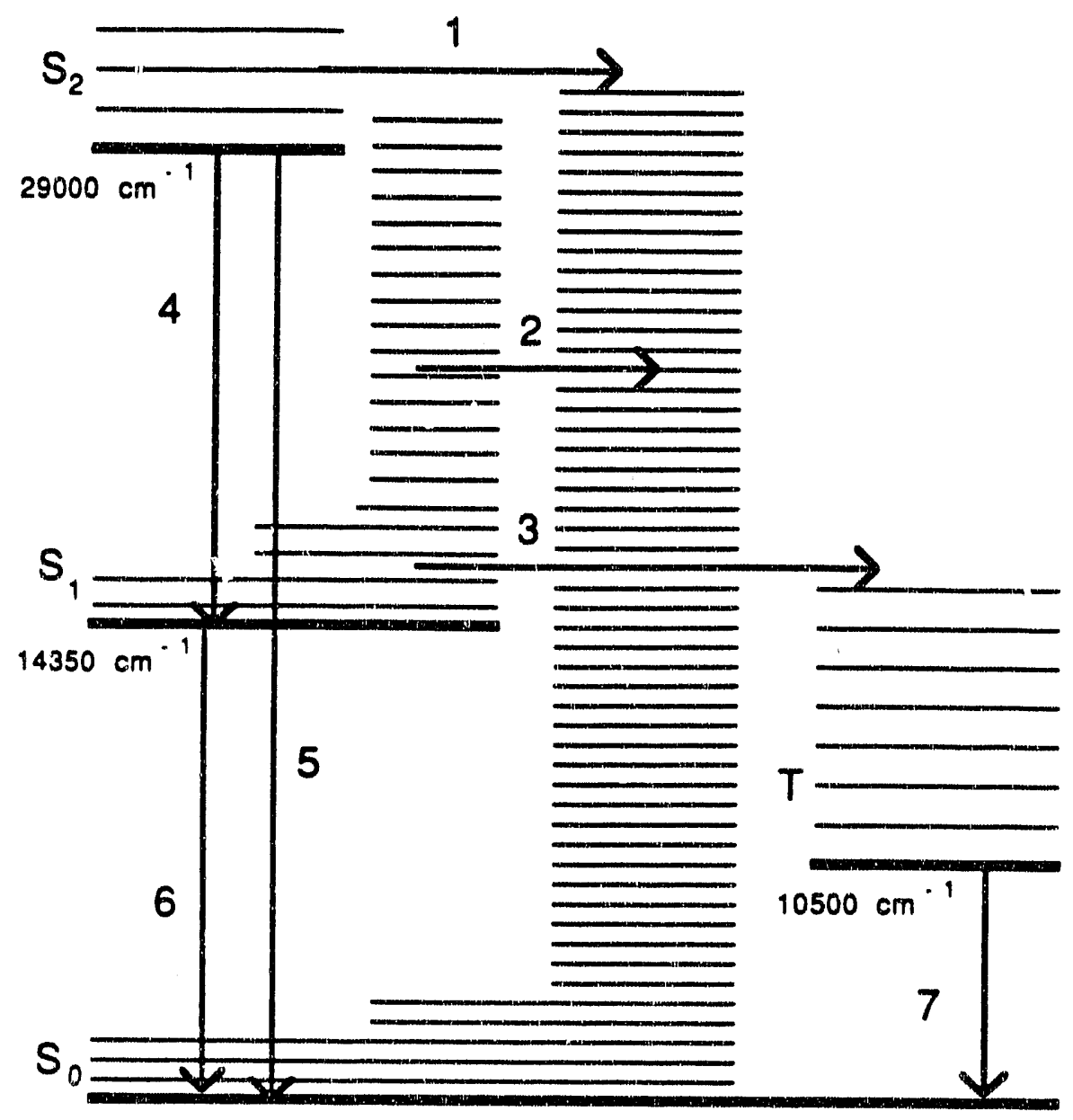

$1 T=4.5 \times 10^{-9} \mathrm{sec}, \phi=0.65$

$2 r=1.9 \times 10^{-12} \mathrm{sec}$

$3 T=5 \times 10^{-7} \mathrm{sec}$

$4 \phi=7 \times 10^{-5}$

$5 \phi=0.031, T=1.6 \times 10^{-9} \mathrm{sec}$

$6 \phi=8 \times 10^{-6}$

$7 \phi=10^{-7}, T=10^{-6} \mathrm{sec}$

Pigure 1.1 Photophysical parameters of azulene energy levels. See Reference 5 and references in text. 
$M^{-1} \mathrm{~cm}^{-1} .^{18}$ The transition strength of the $S_{0}-S_{2}$ transition is approximately ten times larger. ${ }^{6}$ As mentioned above, the fluorescence of the first excited state is very weak and occurs in the 700-800 nm region. ${ }^{7}$ The fluorescence from the second excited state is stronger, fluorescing in the 350-450 $\mathrm{nm}$ region. The fluorescence of $\mathrm{S}_{2}-\mathrm{S}_{1}$ is about 500 times weaker than this, with a quantum yield similar to $s_{1}$ fluorescence. 8 The intensity and wavelengths of these processes are shown in Figure 1 of Reference 8 .

The source of azulene's anomalous fluorescence is its rapid internal conversion time from $s_{1}$ to $s_{0}$. This process has been well studied to accurately measure the ultrafast curve crossing time. The lifetime of the $S_{1}$ state was determined to be $1.9 \pm 0.2$ psec by monitoring the fluorescence from $S_{2}$ as a function of the time delay between two $615 \mathrm{~nm}$ pulses. The first pulse populates $S_{1}$, the second populates the fluorescent $S_{2}$ state as long as population remains in $s_{1} \cdot{ }^{9}$ Thus the signal is sensitive to the lifetime of the first excited state. other work indicates that the internal conversion could be even faster than this, depending upon the internal energy of the azulene. ${ }^{10}$ The internal conversion rate from the second excited state is slow, approximately 4.5 nsec. 11 Therefore internal conversion from $S_{1}$ is the only source of population in the ground state on ultrafast time scales.

\section{1.b Vibrational cooling spectroscopy}


The rapid internal conversion rate into the ground state makes it uniquely possible to study the vibrational sooling dynamics of azulene. Population placed in the first excited state by photoexcitation with a laser pulse moves immediately into the upper vibrational manifold of the ground state. In this manner, a vibrationaldy hot population of ground state azulene molecules is formed with a single laser pulse. The subsequent cooling of the azulene is followed by monitoring vibrational hot band transient absorptions in the $720-760 \mathrm{~nm}$ region. This region corresponds to vibrational hot bands of up to $1200 \mathrm{~cm}^{-1}$ frequencies. The depletion of the hot bands by collisional energy transfer to the solvent as a function of time directly measures the vibrational cooling time. Figure 1.2 diagrammatically illustrates the dynamics occurring throughout the course of the experiment to measure the vibrational cooling rate.

\subsection{Heavy Atom Effect}

The lowest energy triplet state is at approximately 10500 $\mathrm{cm}^{-1}$ with phosphorescence at $9000 \mathrm{~cm}^{-1}$ detected at $77 \mathrm{~K}$ in the absence of $\mathrm{O}_{2}$. The emission lifetime under these conditions is a few microseconds. The extinction coefficient of the $S_{0}-T_{1}$ transition is $10^{-4} \mathrm{M}^{-1} \mathrm{~cm}^{-1} .^{7}$ Because of the existence of this low Iying triplet state, there was concern about intersystem crossing from $\mathrm{S}_{1}-\mathrm{T}_{1}$ interfering with the internal conversion process from $S_{1}-S_{0}$. Rapid intersystem crossing would deplete 


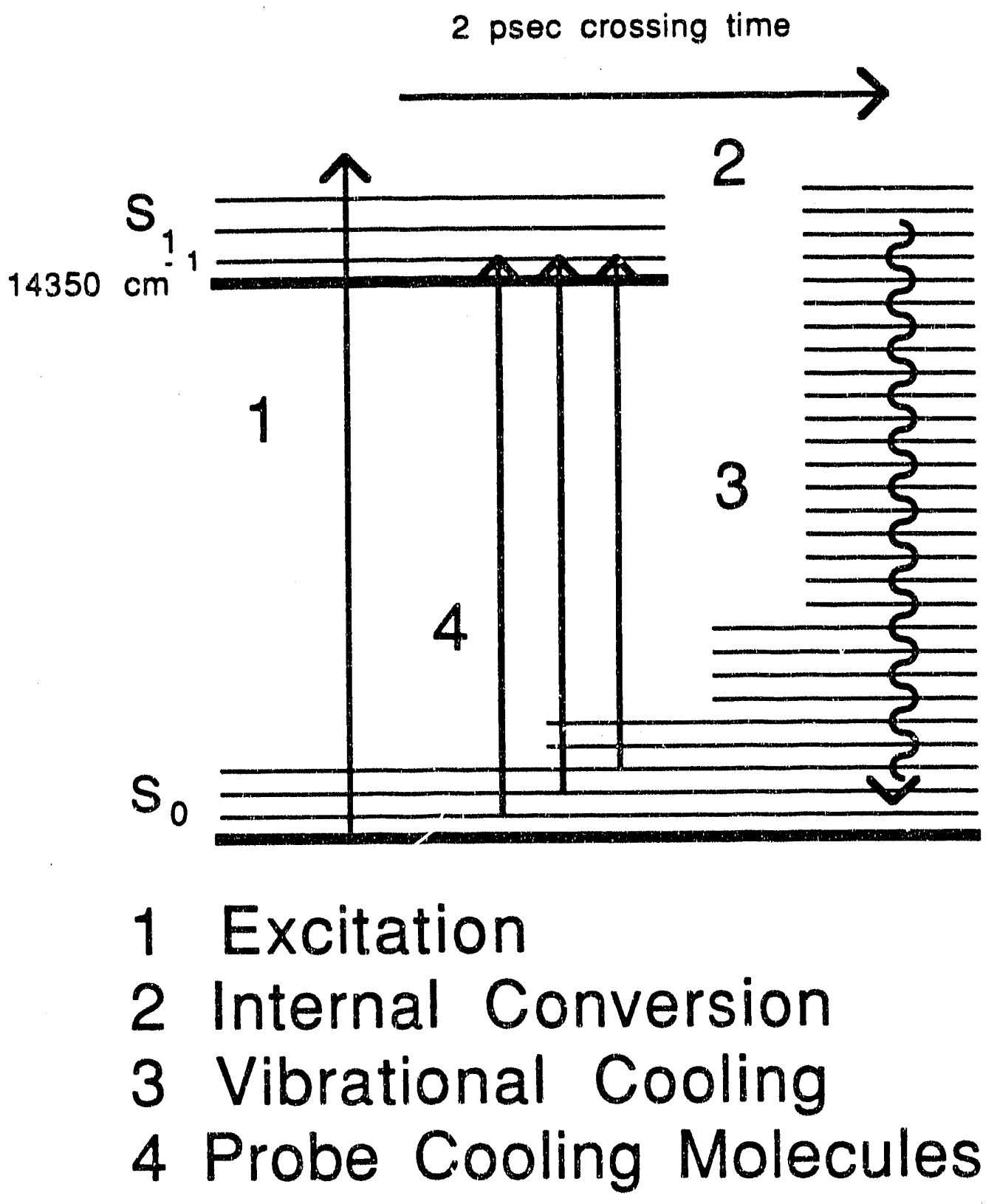

Pigure 1.2 Energy level diagram illustrating vibrational cooling dynamics occurring during experiment. 
the $s_{1}$ population as would the process of internal conversion and subsequent vibrational relaxation. The convolution of these three simultaneous reactions would make the experimental results of energy transfer studies difficult to interpret.

There has been debate in the literature as to the existence of a measurable heavy atom effect on the internal conversion lifetime. ${ }^{12}$ Basically, a heavy atom neax a lighter atom will increase the spin orbit coupling between them. Increased spin orbit coupling facilitates intersystem crossing, a usually forbidden process. ${ }^{13}$

current evidence indicates that there is not a significant heavy atom effect on the internal conversion time. ${ }^{14}$ The intersystem crossing rate from the first excited state to the first triplet state is so slow $\left(2 \times 10^{6} \mathrm{sec}^{-1}\right)^{15}$ that it does not efficiently contribute to the depopulation of $s_{1} \cdot 7,16$ Therefore, the vibrational relaxation dynamics in heavy atom solvents are not due to increased intersystem crossing but due to inherent solute-solvent interactions.

\section{1.d Pressure Dependent Absorption Shifts}

Another concern relevant to this research is the effect of pressure on the absorption spectrum of azulene. A large pressure dependence could affect the vibrational relaxation process and thus the usefulness of comparing data at different densities. Two studies have investigated this pressure dependence. ${ }^{16,17}$ 
The absorption to the first and second excited states was examined in ethanol and pentane up to $5000 \mathrm{~atm}(\approx 75,000 \mathrm{psi})$. In pentane, the first excited state of azulene (labelled ${ }^{1} I_{b}$ in older terminology) does not shift with pressure although the second excited state (labelled ' $B_{b}$ ) does. In ethanol, the first excited state absorption spectrum shifts to the blue about $50 \mathrm{~cm}^{-1}$ over the density range $0.78-0.92 \mathrm{~g} / \mathrm{ml}$ while the second excited state spectrum shift.s by $-200 \mathrm{~cm}^{-1}$. Since noble gases are nonpolar, the solvent environment will resfmble that of pentane, which causes no shift in $S_{1}$ with pressure. ${ }^{16}$

There has also been a study of azulene in plastic films up to $130,000 \mathrm{~atm}(\approx 1,900,000 \mathrm{psi})$. As in the study cited earlier, the absorption spectrum of the second excited state shifts to lower energies with increasing pressure and the first excited state absorption spectrum is only minimally affected by pressure, changing less than $1 \mathrm{~cm}^{-1} / 1000 \mathrm{~atm}$ in the polar film, and $-5 \mathrm{~cm}^{-1} / 1000 \mathrm{~atm}$ in the nonpolar film. ${ }^{17}$ From these results it is obvious that large changes in pressure will not significantly alter the states involved in this experiment.

The chemical structure of azulene $\left(\mathrm{C}_{10} \mathrm{H}_{8}\right)$ is shown in the upper left corner of Figure 1.3. It is a planar, aromatic hydrocarbon. Because azulene is an isomer of naphthalene, one might expect that isomerization between the two species could occur. However, studies indicate that the isomerization of azulene to naphthalene upon photoexcitation in the visible 
region is negligible. ${ }^{18}$

\section{1.e Azulene Absorption Spectrum}

A spectrum of $2 \mathrm{mM}$ azulene dissolved in liquid xenon of density $1.8 \mathrm{~g} / \mathrm{ml}$, reduced density $\rho^{*}=0.57$, at $280 \mathrm{~K}$ is shown in Figure 1.3. This spectrum was taken in the low pressure cell (see section 2.2 for details) using a Cary 13 spectrometer. The spectrum strongly resembles a spectrum of azulene in hexane. There is no spectral shift seen in the xenon solution which indicates that the noble gas solvent does not strongly interact with azulene and perturb the energy levels.

The peaks in the spectrum correspond to a series of vibrational progressions, combinations and overtones of the following three symnetric stretches of similar intensity in excited state azulene: $860 \mathrm{~cm}^{-1}, 1400 \mathrm{~cm}^{-1}, 1560 \mathrm{~cm}^{-1}$. The gas phase spectrum shows three additional modes $\left(385 \mathrm{~cm}^{-1}, 660\right.$ $\left.\mathrm{cm}^{-1}, 1190 \mathrm{~cm}^{-1}\right) .{ }^{19}$ These modes have about half the intensity of the first three modes. ${ }^{20} \mathrm{~A}$ matrix spectrum at $4 \mathrm{~K}$ requires a description incorporating the 12 symmetric modes, excluding $\mathrm{CH}$ stretches. ${ }^{21}$ The peaks in the xenon spectrum are sharper than in hexane, presumably due to the colder temperature of the xenon spectrum.

\section{1.f Mode Analysis of Azulene}

The vibrational spectroscopy of azulene has been well 


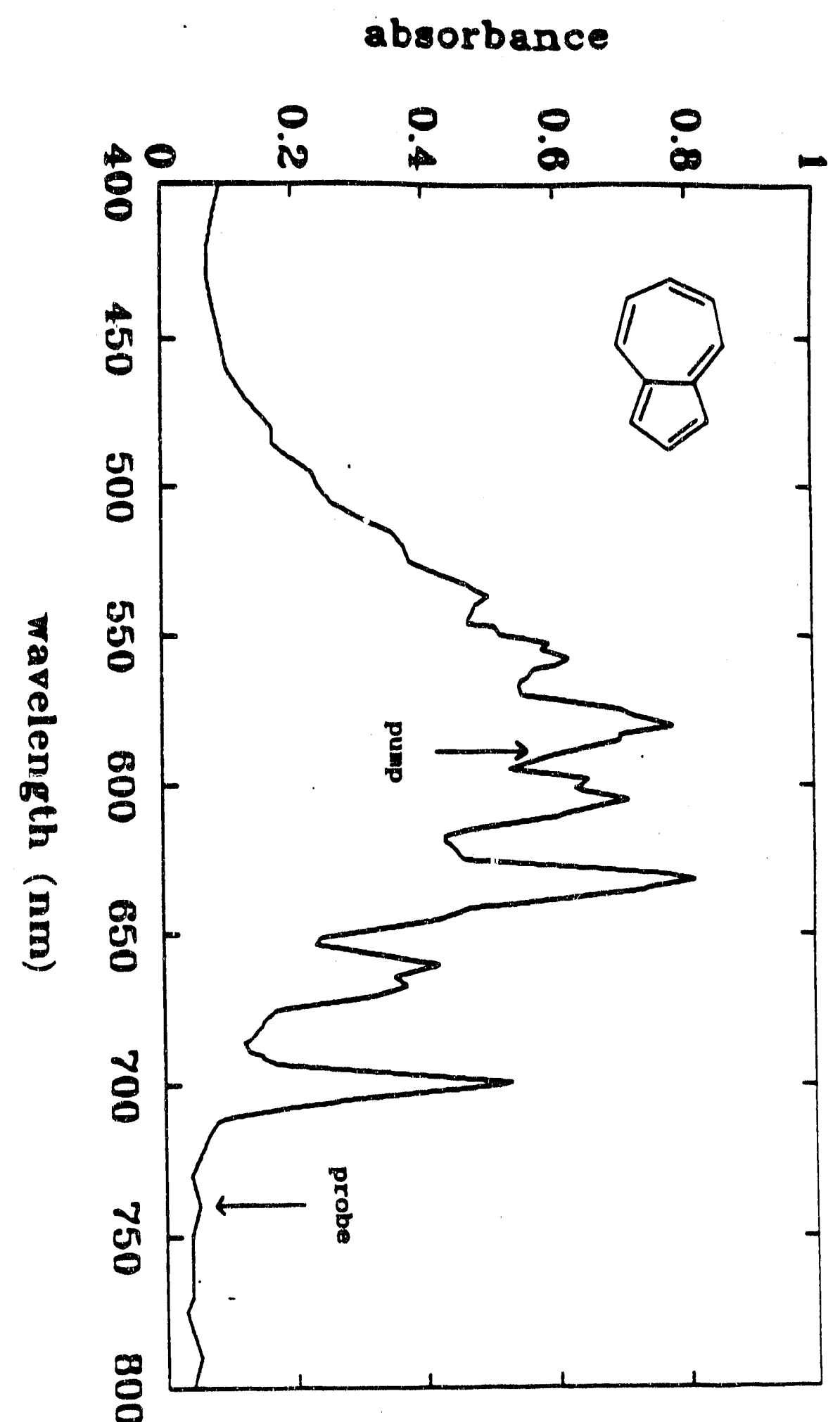

Pigure 1.3 Visible absorption spectrum of $2 \times 10^{-3} \mathrm{M}$ azulene in xenon at $280 \mathrm{~K}, 1.8 \mathrm{~g} / \mathrm{ml}, \rho^{*}=0.57$. 
characterized by infrared and Raman experiments and by theoretical normal mode analysis. These results not only determine the exact frequencies of the vibrational modes but also indicate the activity of modes during the electronic transition and internal conversion. If the active modes are determined for the electronic transition and subsequent internal conversion, these may also be actively involved in vibrational relaxation. At the very least, the modes involved in internal conversion must somehow couple their energy into lower frequency molecular or solvent modes in order to vibrationally relax. Therefore, understanding the vibrational spectroscopy of the molecule is important to understanding vibrational relaxation.

Theoretically, the bridging carbon bond should be artive in the internal conversion process because that bond changes the most between $S_{1}$ and $S_{0}$, contracting about $0.05 \AA$ in $S_{1} .{ }^{22}$ There is also accompanying lengthening along the carbon bonds adjacent to the bridge upon excitation. ${ }^{23}$ The transition between $S_{0}$ and $S_{2}$ causes a less dramatic change in structure, with the azulene expanding slightly in all its bonds. The $\mathrm{C}-\mathrm{H}$ bonds are not affected by the $s_{0}-S_{1}$ transition. ${ }^{22}$ The lack of involvement of the $\mathrm{C}-\mathrm{H}$ stretches in internal conversion is evidenced by the minimal isotope effect on the $s_{1}$ lifetime.? This result will be discussed later in light of the solvent isotope effect on vibrational relaxation seen in this current study, indicating the lack of involvement of these modes in 
vibrational relaxation.

A compilation of the 48 vibrational modes of azulene in the ground state is shown in Table 1.1, as obtained from infrared spectroscopic studies. ${ }^{24}$ The direct correlation of ground state modes with modes in the first excited state is not well determined. There is not only disagreement in the iiterature about the exact frequencies and assignments of modes $^{25}$ but there has also been disagreement concerning the direct assignment of ground and excited state frequencies. ${ }^{21,20,29}$ This is due to the fact that there is some vibronic coupling in the transition to the first excited state (the Dushinsky effect). This prohibits a direct one-to-one correlation of frequencies. ${ }^{21}$ This is particularly noticeable for the $899 \mathrm{~cm}^{-1}$ mode which, according to a well accepted assignment by Hochstrasser and Nyi, has no counterpart in the excited state. ${ }^{20}$ This is unfortunate because this mode has a great deal of $C-C$ single bond character, which corresponds to the single bond character of the bridging carbon bond. ${ }^{24}$ It is also active in the seven membered ring. ${ }^{23}$ Determining how this bond changes upon excitation and relaxation might better indicate how the molecular structure is affected by the relaxation process.

It is well known ${ }^{26,27,28}$ that the totally symmetric stretches are active in the internal conversion process along with some weaker asymmetric stretches. The modes active in promoting the $S_{1}-S_{0}$ internal conversion are the $b_{1}$ modes 1246 
Table 1.1 Ground state vibrational modes of azulene. Tabular data from Reference 24 .

\begin{tabular}{|c|c|c|c|}
\hline Symmetry & Mode Description & \multicolumn{2}{|c|}{ Frequency $\left(\mathrm{cm}^{-1}\right)$} \\
\hline \multirow[t]{5}{*}{$\mathbb{A}_{1}$} & $\mathrm{C}-\mathrm{H}$ stretch & $\begin{array}{l}3091 \\
3059 \\
3002\end{array}$ & $\begin{array}{l}3074 \\
3024\end{array}$ \\
\hline & $\begin{array}{l}\text { C-c (aromatic) } \\
\text { stretch }\end{array}$ & $\begin{array}{l}1638 \\
1443 \\
\end{array}$ & $\begin{array}{l}1580 \\
1392 \\
\end{array}$ \\
\hline & $\mathrm{CCH}$ ip bend & $\begin{array}{l}1294 \\
1054 \\
\end{array}$ & 1117 \\
\hline & $\begin{array}{l}\mathrm{C}-\mathrm{C} \text { (single bond) } \\
\text { stretch }\end{array}$ & 899 & \\
\hline & CCC ring bend & $\begin{array}{l}671 \\
492 \\
\end{array}$ & $\begin{array}{l}559 \\
403 \\
\end{array}$ \\
\hline \multirow[t]{2}{*}{$\mathrm{A}_{2}$} & $\mathrm{C}-\mathrm{H}$ op bend & $\begin{array}{l}1000 \\
795\end{array}$ & 908 \\
\hline & Ring op deformation & $\begin{array}{l}531 \\
163 \\
\end{array}$ & 323 \\
\hline \multirow[t]{2}{*}{$B_{1}$} & $\mathrm{C}-\mathrm{H}$ op bend & $\begin{array}{l}1000 \\
946 \\
721 \\
\end{array}$ & $\begin{array}{l}960 \\
764\end{array}$ \\
\hline & Ring op deformation & $\begin{array}{l}664 \\
311 \\
\end{array}$ & $\begin{array}{l}492 \\
165 \\
\end{array}$ \\
\hline \multirow[t]{4}{*}{$\mathrm{B}_{2}$} & $\mathrm{C}-\mathrm{H}$ stretch & $\begin{array}{l}3083 \\
3007 \\
\end{array}$ & 3044 \\
\hline & $\begin{array}{l}\text { C-C (aromatic) } \\
\text { stretch }\end{array}$ & $\begin{array}{l}1694 \\
1580 \\
\end{array}$ & $\begin{array}{l}1479 \\
1453 \\
\end{array}$ \\
\hline & $\mathrm{CCH}$ ip bend & $\begin{array}{l}1301 \\
1151 \\
970 \\
\end{array}$ & $\begin{array}{l}1205 \\
1007\end{array}$ \\
\hline & CCC ring bend & $\begin{array}{l}721 \\
559 \\
\end{array}$ & $\begin{array}{l}593 \\
478 \\
\end{array}$ \\
\hline
\end{tabular}

op $=$ out of plane, ip = in plane 
$\mathrm{cm}^{-1}, 1349 \mathrm{~cm}^{-1}$ and $1448 \mathrm{~cm}^{-1}$. The modes which accept the energy of internal conversion are the symmetric stretches 1469 $\mathrm{cm}^{-1}, 2570 \mathrm{~cm}^{-1}$ and $1584 \mathrm{~cm}^{-1}$ as well as their overtones. The promoting modes are $\mathrm{CCC}$ and $\mathrm{CCH}$ deformations and stretching modes delocalized over the whole molecule. The accepting modes are similar deformation modes with CC stretching character as well. 27 The most active mode is presumed by some $^{26}$ to bs the $1580 \mathrm{~cm}^{-1}$ mode because it has the most intensity along the bridging carbon stretch. Others ${ }^{27}$ believe that the $1448 \mathrm{~cm}^{-1}$ mode is most important. studies from gas phase indicate that the low frequency, out of plane, modes which change dramatically between $s_{0}$ and $s_{1}$ are important in the internal conversion. ${ }^{28}$ All agree that the skeletal CC symmetric stretches between $1300 \mathrm{~cm}^{-1}$ and $1700 \mathrm{~cm}^{-1}$ are actively involved in and vibrationally excited by the $s_{1}-s_{0}$ internal conversion. The motions associated with these modes are shown in Figure 1 of Reference 2.3.

However, activity in a vibrational mode during internal conversion does not mean that that mode will necessarily be the most important in the process of vibrational cooling. The accepting modes mentioned above may be important ir that they are high frequency and can accept the large amount of energy $\left(\approx 17,000 \mathrm{~cm}^{-1}\right)$ that is placed into the ground state upon internal conversion. The coupling between the ground and first excited state is strong and there is additional coupling to higher energy states such as $\mathrm{S}_{4}{ }^{29}$ which could induce 
activity in a number of vibrational modes. There is also a large change in the permanent dipole $(\mu=1 D)$ of $\Delta \mu=2 D$ over a dipole length of $0.4 \AA$ which will activate many different modes. ${ }^{29}$ Finally, there are two very low frequency modes ( $\approx$ $160 \mathrm{~cm}^{-1}$ ) present in azulene and at least five modes below 400 $\mathrm{cm}^{-1}$ which could efficiently couple vibrational energy to the solvent. These low frequency modes are not necessarily coupled to the $s_{0}-s_{1}$ transition ${ }^{20}$ but may be important in vibrational relaxation because they are more accessible to coupling with low frequency solvent modes, which for noble gas solutions are on the order of $50 \mathrm{~cm}^{-1} \cdot 30$ Therefore, although higher frequency skeletal modes may be excited upon internal conversion, the low frequency modes are probably responsible for draining energy to the solvent. Without mode specific detection (e.g. IR detection), we can not know exactly which modes are relaxing.

\subsection{Experimental Studies of Energy Transfer in Azulene}

The vibrational cooling of azulene has been studied under a wide range of conditions. This work has largely been done in the gas phase or in matrices. Some work has investigated the relaxation of azulene from its second excited state, because $S_{2}$ is more fluorescent, which facilitates study. others studied dynamics and spectroscopy in both the first and second excited state. Only one group has investigated the liquid phase relaxation of azulene to date. ${ }^{38}$ The only study 
to examine the density dependence of the ground state relaxation of azulene is the present one.

\section{2.a Gas Phase Studies of Energy Transfer in Azulene}

Many groups have investigated the process of energy transfer between azulene and buffer gases in the gas phase. clean photophysics, accessible excited states and minimal interfering fluorescence facilitate these experiments. Basically, energy transfer has been studied by two groups using two different experimental techniques: IR fluorescence ${ }^{31,32}$ and hot UV absorption. 33

Briefly, these two experiments measure gas phase energy transfer between hot ground state azulene molecules and buffer gases using slightly different measurement techniques to probe the azulene in time. Azulene in various gas mixtures is excited to the first excited state with $\approx 17,000 \mathrm{~cm}^{-1}$ of internal energy. Rapid internal conversion produces hot ground state azulene and the cooling of these molecules is detected in time. one detection scheme uses infrared fluorescence from the $\mathrm{C}-\mathrm{H}$ stretches in azulene around $3000 \mathrm{~cm}^{-1}$ to detect the depopulation rate of the modes upon vibrational cooling." The other detection scheme excites the hot ground state azulene to a fluorescent excited state $\left(S_{2}\right)$ and collects the fluorescence as a function of time. 33

studies show that bulk thermally heated molecules yield absorption spectra that resemble those of molecules rapidy 
heated with a laser pulse, as in the experiments just described. ${ }^{34}$ The absorption changes in the hot molecule indicate the difference in population. The change in absorption is monitored in time to determine the relaxation of this population, thus the energy transfer rate.

The results of these experiments provide information about the collisions between azulene and surrounding molecules. These results will become particularly important later on in comparing gas phase energy transfer theories with liquid phase relaxation processes. The general trend seen in these experiments is that the amount of energy transferred per collision between the azulene and collider $(\Delta E)$ is linearly dependent upon the internal energy of the azulene $(\langle E\rangle)$. The general relaxation was very close to an exponential decay, and was modelled as such for ease, though this did cause some problems in the calculations. 32 The amount of energy transferred depends on the collider and also the temperature of the bath gas. The results of these gas phase energy transfer studies have been listed in Table 1.2 (results from Reference 33). This table plots the energy transferred per collision $(\Delta E)$ at three different values of available internal energy $\left(\langle E\rangle-\left\langle E_{T}\right\rangle\right.$ where $\left\langle E_{T}\right\rangle$ is the equilibrium internal energy).

This information is useful for comparing the vibrational cooling dynamics of azulene in gases and compressed gases. The excitation wavelengths in the gas and compressed gas 
Table 1.2 Azulene-buffer gas energy transfer values ( $\Delta E$ in $\left.\mathrm{cm}^{-1}\right)$ at three different internal energies $\left(\langle E\rangle-\left\langle E_{T}\right\rangle\right.$ in $\left.\mathrm{cm}^{-1}\right)$. (Tabular data from Reference 33 ).

\begin{tabular}{|l|l|l|l|l||}
\hline \multicolumn{2}{|c|}{} & \multicolumn{4}{|c|}{$\Delta E$ for $\langle E\rangle-\left\langle E_{T}\right\rangle$} & $\left(\mathrm{cm}^{-1}\right)$ \\
\hline Solvent & Temp. (K) & 5000 & 10000 & 15000 \\
\hline Ne & 298 & 60 & 100 & 130 \\
\hline Ar & 298 & 70 & 120 & 170 \\
\hline Kr & 298 & 60 & 120 & 160 \\
\hline Xe & 298 & 50 & 120 & 170 \\
\hline Ne & 410 & 35 & 65 & 95 \\
\hline Ar & 600 & 50 & 80 & 85 \\
\hline Kr & 670 & 40 & 70 & 100 \\
\hline $\mathrm{Xe}$ & 830 & 40 & 80 & 110 \\
\hline
\end{tabular}


experiments are similar. Therefore, the same amount of energy in the molecule is present and must be dissipated to the suxrounding molecules. Despite the difference in detection systems to measure energy transfer, the results will be similar enough to provide information about vibrational cooling of azulene.

One might guess that information on $\Delta E$ for azulene and solvent would lead directly to a time for vibrational cooling. Given the total internal energy of the molecule $\left(17,000 \mathrm{~cm}^{-1}\right)$ and $\Delta E$, one could then calculate the number of collisions needed to totally deactivate the molecule. From the information in Table 1.2 the number of collisions to deactivate azulene to an equilibrium energy of $2000 \mathrm{~cm}-1$ (1000 $\mathrm{cm}^{-1}$ of internal energy at $300 \mathrm{~K}$ plus $1000 \mathrm{~cm}^{-1}$ of hot band energy) can be determined. Xenon and krypton require approximately 280 and 240 collisions respectively. Considering the decay time of azulene in these solvents, the collision frequencies calculated are quite reasonable, approximately $2 \mathrm{psec}^{-1}$. Unfortunately, a small alteration in the value of $\Delta \mathrm{E}$ at low energies around $5000 \mathrm{~cm}^{-1} \mathrm{can}$ radically altex the number of collisions needed to deactivate the azulene and this distorts the calculated collision frequency. The gas phase data on energy transfer cannot be directly applied in this manner to the current azulene results to predict vibrational relaxation times. 


\section{2.b Theoretical Studies of Energy Transfer in Azulene}

The gas phase studies of energy transfer between azulene and other collider molecules provide a basis for a theoretical investigation into the relaxation dynamics of this molecule. 35 The exponential form of the relaxation that is seen in the gas phase studies (and in this current study) are of particular interest. That exact exponential relaxation should occur in a complicated molecule like azulene was surprising. Generally, there are a number of limiting conditions which must occur for exact exponential relaxation to occur, such as strong collisions and simple energy flow pathways. These conditions are usualiy only met for simple diatomics and poiyatomics when single level relaxation is monitored. However, the azulene experiments showed a linear relationship between the average energy transferred upon collision and the total average energy of the azulene. This relationship defines the necessary condition for exponential relaxation. This does not necessarily mean that the population of the molecular modes relaxes exponentially, just that the average energy does.

Exponential relaxation was not seen in studies of the vibrational relaxation of molecular iodine, a much simpler molecule. ${ }^{36}$ The difference between these two molecules may simply be due to anharmonicity in the excited vibrational modes. Highly excited iodine is in a strongly anharmonic potential region as it starts to relax whereas a largely 
harmonic potential is sampled in the azulene since intramolecular energy redistribution in the molecule causes many modes to be excited. This leads to a difference in the collisions which occur and the amount of energy transferred in a collision.

This exponential behavior illuminates the nature of the collision between azulene and the collider. The two collision partners must behave in an ergodic or near-ergodic manner. Unfortunately, the above condition may be met with either strong collision of the azulene and solvent or weak collisions, the latter condition affecting only a small subset of modes in the azulene. Therefore, this result can not distinctly illuminate the details of the azulene-solvent collision. This analysis concludes that only approximate exponential relaxation could be experimentally measured, given the inherent error in the experiments.

\subsection{Crystal studies of Energy Transfer in Azulene}

Study of the Raman active modes in the $s_{0}-s_{1}$ transition also provides information about the vibrational relaxation time of some of these Raman active modes in the first excited state. Raman spectroscopy of an azulene doped naphthalene crystal at $2 \mathrm{~K}$ determined vibrational relaxation times for these four symmetric stretches in $\mathrm{S}_{1}: 384 \mathrm{~cm}^{-1}, 665 \mathrm{~cm}^{-1}, 856$ $\mathrm{cm}^{-1}, 912 \mathrm{~cm}^{-1}$. These relaxation times varied between $1.3 \mathrm{psec}$ and $10.8 \mathrm{psec}$, which is longer than the internal conversion 
time. Of the modes studied, it appears that the $384 \mathrm{~cm}^{-1}$ mode is not coupled to the relaxation of other modes. The higher frequency modes are only well coupled to the energy levels within approximately $150 \mathrm{~cm}^{-1}$ (well coupled is defined as populated with at least $10 \%$ of the pumped level population).$^{20}$ These results indicate that little relaxation occurs in $s_{1}$ before internal conversion occurs so that the population that enters the ground state contains almost all the energy of the laser pulse. This study also shows how relaxation may occur among vibrational levels in the ground state. The low frequency modes seem surprisingly inactive in the relaxation process, perhaps because higher frequency modes in azulene would be resonant with modes in the naphthalene crystal. It is questionable whether the high frequency modes would be as active in a noble gas media, which lacks resonance with the solute.

\section{2.d Matrix Studies of Energy Transfer in Azulene}

The experiments involving matrix studies ${ }^{37}$ examined a temperature and solvent mass effect seen on the vibrational relaxation of azulene in the second excited state. The fluorescence from $S_{2}(v=0)$ is monitored to determine the lifetime of the excited population. In a cryogenic Xe matrix, this relaxation time is about 50 picoseconds. There is a significant effect when lighter noble gas atoms are used in the matrix, with lighter noble gases causing faster 
relaxation. The effect is two to three times stronger than the effect that is seen in this current study, leading to faster relaxation times in noble gas matrices than noble gas liquids. This is due to the fact that phonon modes of a crystal are lower in frequency than in a liquid. Therefore, the mismatch in frequencies between azulene and a xenon crystal is much worse than azulene in a neon crystal.

Additionally, the temperature of the Xe matrix was changed over a small range of temperature, $4 \mathrm{~K}$ - 50K. Over this range, there was no effect on the relaxation rate. They mention that there might be the possibility of a bottleneck in the vibrational levels of $S_{2}$.

It is not clear whether these results concerning $s_{2}$ can be used to describe the ground state relaxation rates seen in the present work. However, if there really is a bottleneck in the second excited state of azulene, this could imply a similar result for the ground state. The geometry of the ground state and the second excited state are somewhat similar. ${ }^{22}$ If the geometry of the second excited state can induce a decoupling of some of the modes in the azulene, then this could also occur in the ground state. This is a possibility that should not be overlooked in the analysis of the study of azulene ground state dynamics.

\section{2.e Liquid Study of Energy Transfer in Azulene}

Kaiser and coworkers ${ }^{38}$ initially examined the 
vibrational cooling dynamics of ground state azulene in liquids. The experimental system used a 5 psec excite pulse at $527 \mathrm{~nm}$ and probe pulse around $735 \mathrm{~nm}$. Immediately upon photoexcitation, an absorption spectrum was seen, resembling that of a hot azulene molecule at 1300K. A portion of this absorption spectrum was monitored in time to give a cooling rate. The resulting cooling time measured of azulene in $\mathrm{CCl}_{4}$ was $40 \pm 15$ psec.

Given the relative simplicity of azulene and the experimental. ease with which one can conduct vibrational relaxation studies, a systematic study of the relaxation of azulene in many different solvents was initiated. By examining a range of different solvent systems, the relaxation rate could be reexamined. The effect of solvent on this value could be determined, illuminating the microscopic effect of energy transfer between molecules in solution. 


\section{References for Chapter 1}

1. S.P. McGlynn, T. Azumi, and M. Kinoshita, Molecular Spectroscopy of the Triplet state, (Prentice-Hall, Inc., New Jersey, 1969), p. 7 .

2. S. Murata, C. Iwanaga, T. Toda, and H. Kokubun, Chem. Phys. Lett. 15, 152 (1972).

3. D. Huppert, J. Jortner, and P.M. Rentzepis, J. Chem. Phys. 56, 4826 (1972).

4. Michael Beer and H.C. Longuet-Higgins, J. Chem. Phys. 23, 1390 (1955).

5. For a review of azulene spectroscopy, see Gregory $D$. Gillispie and E.C. Lim, J. Chem. Phys. 68, 4578 (1978) and Klaus Gustav and Michael Storch, Int. J. Quantum Chem. 38, I (1990).

6. G. Binsch, E. Heilbronner, R. Jankow, and D. Schmidt, Chem. Phys. Lett. 1, 135 (1967).

7. P.M. Rentzepis, Chem. Phys. Lett. 3, 717 (1969).

8. Dieter Klemp and Bernhard Nickel, Chem. Phys. Lett. 130, $493(1986)$.

9. E.P. Ippen, C.V. Shank, and R.L. Woerner, Chem. Phys. Lett. $46,20(1977)$.

10. Sudhir K. Kulkarni and Jonathan E. Kenny, J. Chem. Phys. 89, 4441 (1988).

11. Y. Hirata and E.C. Lim, J. Chem. Phys. 69, 3292 (1978).

12. E. Drent, G. Makkes van der Deijl, and P.J. Zandstra, Chem. Phys. Lett. 2, 526 (1968).

13. S.P. McGlynn, T. Azumi, and M. Kinoshita, Molecular Spectroscopy of the Triplet state, (Prentice-Hall, Inc., New Jersey, 1969), pp. 40-43.

14. Hans J. Griesser and Urs P. Wild, Chemical Physics, 52, 117 (1980); E.P. Ippen, C.V. Shank, and R.L. Woerner, Chem. Phys. Lett. 46, 20 (1977).

15. H.J. Kray and B. Nickel, Chem. Phys. 53, 235 (1980).

16. Dean J. Mitchell, Harry Drickamer, and Gary B. Schuster, J. Amer. Chem. Soc. 99, 7489 (1977). 
17. W.W. Robertson and A.D. King, Jr., J. Chem. Phys. 34, 1511 (1961).

18. M. Comtet and H.D. Mettee, Mol. Photochem. 2, 63 (1970).

19. Ranty Liang, Ott.o Schnepp, and Arieh Warshel, Chem. Phys. 34, 17 (1978).

20. R.M. Hochstrasser and C.A. Nyi, J. Chem. Phys. 70, 1112 (1979).

21. O. Brafman, C.K. Chan, B. Khodadoost, J.B. Page, and C.T. Walker, J. Chem. Phys. 80, 5406 (1984).

22. Klaus Gustav and Michael Storch, Intl. J. Quantum Chem. $38,25(1990)$.

23. Francesco Zerbetto and Marek Zgierski, Chem. Phys. 110, $421(1986)$.

24. O. Gebhardt, Acta Chem. Scand. 27, 1725 (1973).

25. Kieran F. Lim and Robert G. Gilbert, J. Chem. Phys. 84, $6129(1986)$.

26. Hans J. Griesser and Urs P. Wild, Chem. Phys. 52, 117 (1980).

27. Klaus Gustav and Michael Storch, Intl. J. Quantum Chem. 38, 1 (1990).

28. Toshinori Suzuki and Mitsuo Ito, J. Phys. Chem. 91, 3537 (1987).

29. G.J. Small and S. Kusserow, J. Chem. Phys. 60, 1558 (1974).

30. J.K. Brown, D.J. Russell, D.E. Smith, and C.B. Harris, Revue Phys. Appl. 22, 1787 (1987); J. Keenan Brown, Ph.D. thesis, Molecular Dynamics Simulations of Simple Liquid Phase Chemical Reactions, University of California - Berkeley, Berkeley, CA 1987.

31. Michel J. Rossi, Jack R. Pladziewicz, and John R. Barker, J. Chem. Phys. 78, 6695 (1983).

32. Jichun Shi and John R. Barker, J. Chem. Phys. 88, 6219 (1988).

33. H. Hippler, B. Otto, and I. Troe, Ber. Bunsenges. Phys. Chem. 93, 428 (1989). 
34. L. Brouwer, H. Hippler, L. Lindemann, and J. Troe, J. Phys. Chem. 89, 4608 (1985).

35. Wendell Forst, Chem. Phys. 147, 241 (1990).

36. M.E. Paige and C.B. Harris, Chem. Phys. 149, 37 (1990).

37. J.B. Hopkins and P.M. Rentzepis, Chem. Phys. Lett. 142, 503 (1987); Chem. Phys. Lett. 117, 414 (1985).

38. W. Wild, A. Seilmeier, N.H. Gottfried, and W. Kaiser, Chem. Phys. Lett. 119, 259 (1985). 
Chapter 2: EXPERIMENTAL

\subsection{Picosecond Laser system}

All experiments were performed with a synchronously pumped dye laser amplified with the second harmonic (532 nm) of a $10 \mathrm{~Hz}$ Nd:YAG laser in a three stage dye cell amplifier. This system produces a $590 \mathrm{~nm}, 1$ psec pulse at $10 \mathrm{~Hz}$ with $1 \mathrm{~mJ}$ pulse energy and approximately $10 \%$ noise shot-to-shot.' Figure 2.1 shows the schematic of the laser system. $40 \%$ of the pulse excites the azulene to the first excited state, the remainder is focussed into a water or acetone cell to generate the probe frequencies via continuum generation. A specific probe wavelength is selected with a $10 \mathrm{~nm}$ bandpass filter. The probe pulse is spatially filtered to improve the focussing and spatial quality of the beam.

Approximately $4 \%$ of the probe is reflected off each of 2 silica flats, $1 / 2$ " thick to facilitate physical selection of the front reflection. These reflections form the signal and reference probe lines. The signal and reference probe beains pass through a 1 cin pathlength cell of azulene solution and is focussed into an EG\&G DT-110 photodiode. No reference cell was used for the experiments involving the pressure cell. The sample probe beam is focussed with a $25 \mathrm{~cm}$ lens into the sample cell to $2 \mathrm{~mm}$ in diameter. The excite beam can then be focussed independently with a $50 \mathrm{~cm}$ lens and overlapped with the probe spot. The sample was $45 \mathrm{~cm}$ from the excite 


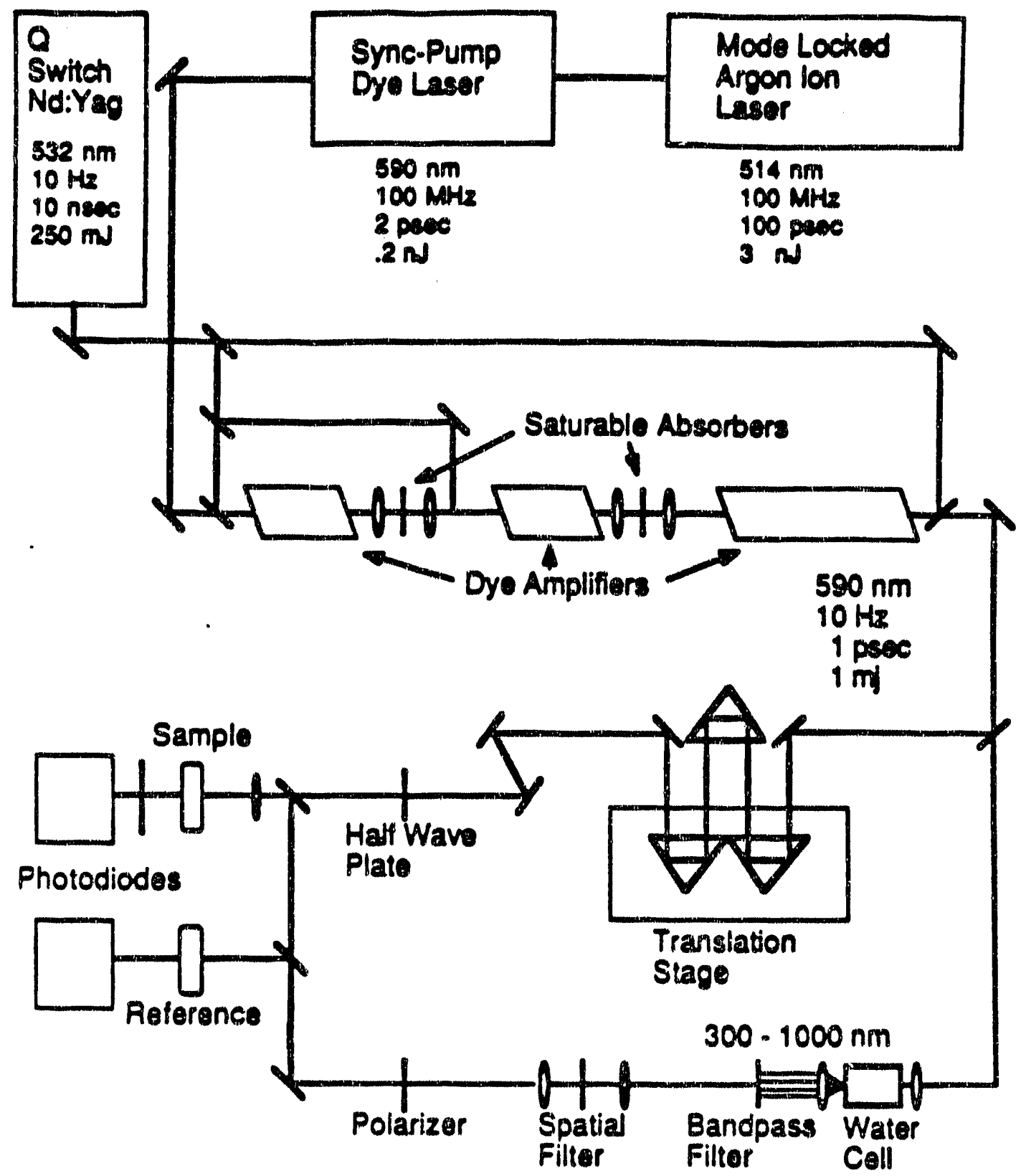

Iigure 2.1 Picosecond laser system and transient absorption apparatus. 
focussing lens, which made the excite spot smaller than the probe. The excite focus was limited by degradation of the azulene. Although a collinear arrangement was initially used to simplify the overlap process, the off angle $\left(6^{\circ}\right.$ from normal) configuration allows tighter focussing and thus larger signal. There was no difference in the time behavior of the transient absorption signal due to this change in configuration and therefore the off angle configuration was used for all experiments. The excite lens was placed on an xyz translation stage to enable adjustment of the overlap for maximal signal size once the sample was in place. After the sample, the probe beam is focussed into a DT-110 photodiode. Identical glass filters are placed before the photodiodes to attenuate the light before collection as well as to block scattered light from the excite beam (Schott RG-630).

The gated photodiode signal was integrated with a Lecroy 10 bit $A / D$ converter interfaced to a personal computer. The reference and sample signals were ratioed and then averaged to remove fluctuations in the probe intensity. This allowed detection of signals down to $0.2 \%$ change in absorbance, averaging 20 laser shots in a single scan. This process was repeated at various positions of 1 meter translation stage with $10 \mu \mathrm{m}$ resolution (Klinger), which changes the pathlength of the excite relative to that of the probe. In this manner, the time behavior of the azulene dynamics was mapped out. Data collection took place on both the forward and backward 
motion of the stage, thus minimizing any effects due to long time background signal drift. Retroreflectors were used to make four passes with the excite light on the stage, giving a minimum time step of $0.133 \mathrm{ps} / \mathrm{step}$.

\subsection{Polarization Control Experiments}

In all the experiments, a vertical polarizer was placed in the probe pulse after spatial filtering while the excite pulse was rotated to the magic angle $\left(54.7^{\circ}\right)$ with a half waveplate. ${ }^{2}$ A vertical polarizer was occasionally used in the excite pulse since it is not completely vertically polarized. However, there was no visible difference in scans taken with and without a polarizer in the excite. When no polarizers or waveplates were used in the experiment, a long time decay of approximately one hundred picoseconds was seen in addition to the rapid decay attributed to vibrational cooling. It is presumed that this decay is due to rotational diffusion of the azulene, as it fits the general decay time for rotational diffusion of large molecules. 3,4 The magic angle pump-probe configuration eliminates this long time component.

Three types of optical cells were used to study the azulene system: a $1 \mathrm{~cm}$ path length glass cuvette, a home built $1 \mathrm{~cm}$ path length pressure cell with $2 \mathrm{~mm}$ thick unoriented sapphire windows (Melles Griot), hereafter called the "low pressure cell", and a homebuilt $1 \mathrm{~cm}$ path length pressure cell with $1 \mathrm{~cm}$ thick oriented (in the direction of the $c$ axis) 
sapphire windows (Union Carbide), hereafter called the "high pressure cell". Initially, it was feared that the sapphire, especially under pressure, might distort the polarization of the pump and probe pulses. Sapphire is notorious for its birefringence and pressure might worsen this effect.5 Therefore, numerous control tests were conducted to determine any effect on the experimental results.

resting the unoriented sapphire windows' effect on polarized light, one sees a $30^{\circ}$ rotation in the polarization of the light but little birefringence. The effect of oriented sapphire at room pressure and at 30,000 psi of argon on a vertically polarized beam perpendicular to the windows was then tested. Two vertical polarizers were placed before and after the cell. The amount of light through the cell and polarizers was collected by a photodiode. There was no change in the signal size before or after pressurization. In addition, a horizontal polarizer after the cell completely extinguished the vertically polarized beam sent into the cell. This indicates that oriented sapphire will not distort the polarization of light even under high pressure.

In the off angle configuration of the pump and probe beams, there still may be a problem with depolarization of the beam, since only one beam can truly be along the (0001) c axis of sapphire and thus remain undistorted. In the off angle configuration, the windows do change the polarization of the incoming light somewhat more than in the collinear 
configuration. The effect is greater for the excite beam than the probe beam, perhaps because the angle off the normal is larger. However, for a well polarized excite beam, depolarization effects are lower than $50 \%$ and a distinctly vertical component remains in the beam. For a poorly polarized excite beam, the depolarization effects are stronger. There does not appear to be a strong birefringence effect in oriented sapphire, as has been noted by other researchers. ${ }^{5}$

Finally, two identical experiments were done using a sample of azulene in the off angle configuration. In one case, the sample was a cuvette of the azulene solution; in the other case, the same cuvette of the solution with $1 \mathrm{~cm}$ oriented sapphire windows on either side of the cuvette. The resulting decay times from the two experiments were identical, indicating no effect on the data due to the sapphire windows, other than slightly reducing the signal size due to sapphire high reflectance ( $8 \%$ per surface in the visible region).$^{6}$

In conclusion, data with and without the sapphire windows, with oriented and non-oriented sapphire, in off angle and collinear arrangements, with and without a vertical polarizer in the excite path are all essentially identical when compared. Thus one can conclude that only a small portion of the light is becoming cistorted by sapphire windows in the off angle configuration or that both the pump and probe have their polarization rotated the same amount, leaving the 
relative angle between the two intact.

\subsection{High Pressure System}

A custom made high pressure cell, capable of 100,000 psi (7000 atm) pressure to compress noble gases, was used as the sample container for most of the experiments involving compressed gases as solvents. Much of the information concerning the details of this system can be found elsewhere. ${ }^{7}$ The system can be described in three parts; a high pressure cell with attached temperature regulator, a moderate and high pressure generation system and a vacuum system for gas handling.

Initially, the whole system is evacuated before pressurizing. This is accomplished with a mechanical vacuum pump and liquid nitrogen cold trap. The solvent gas enters the system via a small ( $<1$ liter) source tank in which the gas of choice had been previously condensed. Krypton and xenon gases were all 99.9998 pure, the Freon-13 gas $\left(\mathrm{CClF}_{3}\right)$ was 99.0\% pure (Matheson). This tank provides 500-1000 psi input pressure to a two stage diaphragm compressor (Newport Scientific), which can reach gas pressures of up to 20,000 psi. At this point, a valve can direct the gas directly into the low pressure cell to do experiments below $1000 \mathrm{psi}$.

Alternatively, the moderate pressure side of the system can be sealed off and the gas directed into a pressure intensifier (Nova Swiss) to increase the gas pressure. Before 
raising the pressure, a few hundred psi of gas are let in the cell, to make a low pressure seal and avoid air leaking into the evacuated cell. Higher gas pressures will dissolve the sample. With an intensification ratio of approximately 15:1, this intensifier was used to obtain pressures of many thousand psi. In this experiment, the maximal pressure used was under $40,000 \mathrm{psi}$, although the system can reliably reach $85,000 \mathrm{psi}$. The gas at its final pressure is then let into the sample cell.

The sample cell was custom made of a titanium alloy, to prevent corrosion from a sample used previously in this system. It is very similar to the high pressure cell produced by Nova Swiss. The window assembly is held in place by means of a mushroom plug and an endcap screwed into the cell body. The windows provide $6 \mathrm{~mm}$ clear aperture. By means of a torque wrench, the endcaps are tightened reproducibly to 300 in-lbs each time to minimize the event of leakage. A teflon gasket seals the windows up to $1000 \mathrm{psi}$. The sealing of the mushroom plugs occurs at $10,000 \mathrm{psi}$. To obtain pressures in between these values, the cell is first pumped up to $10,000 \mathrm{psi}$ and then slowly depressurized to the desired pressure. However, neither method will seal the cell between approximately 5000 and $1000 \mathrm{psi}$. The sample cell contained azulene and a micro stirbar, at least $2 \mathrm{~mm} \times 7 \mathrm{~mm}$ or $1.5 \mathrm{~mm} \times 8 \mathrm{~mm}$ in size, which had been loaded previous to pressurization. Smaller stirbars, weighing less than 0.05 grams, float in the high density 
solutions. A thermally regulated alcohol or glycol solution flowed through copper coils thermally glued to the outside of the cell to maintain the cell temperature to within $1^{\circ} \mathrm{C}$. Due to the cell's size, each experimental condition was allowed to equilibrate for one hour before data collection began. Upon completion of an experiment, the cell is incrementally depressurized by toggling two valves to minimize the chance of window failure. The gas is condensed in the source tank again, returning by means of a separate route to minimize azulene contamination of the pumps and gas lines.

As a reminder to others, precautions must be taken when working with highly compressed gas. The work released when these volumes of compressed gas expand are on the order of $15,000 \mathrm{~J}$, which would move á 1 gram mass at the speed of a bullet. Even at low pressures, component failure yields a loud gunshot noise with the potential for ear damage. Workers wear ear protection when any section is pressurized. Double shields of 1/2" Lexan (a.k.a. bulletproof glass) along the window axis protect the surroundings from the results of catastrophic window failure. 1/2" Lexan also encases the cell body and all pressure gas lines. Safety should always be a consideration when pursuing these types of experiments.

\subsection{Sample Preparation}

As previous researchers have found, azulene is not always pure enough for experiments right out the bottle. Although 
the stated purity of the azulene is about $99 \%$ (Aldrich), most researchers purify their compound first. ${ }^{8,10}$ Two purification schemes were used after photoproduct production was detected during some early experiments. In the first purification process, azulene is dissolved in pentane and separated by column chromatography. ${ }^{8}$ The pentane is then evaporated. The remaining compound is then sublimed at about $10 \mathrm{mTorr}, 30^{\circ} \mathrm{C} .9$ Higher temperatures degrade the azulene. The second technique used was simply to sublime azulene as it comes from the manufacturer. ${ }^{10}$ Mass spectrometry confirmed $>99 \%$ purity of the azulene after sublimation. Both techniques eliminatea spurious signals and reduced photoproduct production. Data from both samples of purified azulene were identical.

Care must be taken when evacuating the cell loaded with the azulene sample. Azulene is a volatile aromatic, with an odor of naphthalene and a vapor pressure of approximately 10 mTorr at room temperature. ${ }^{11}$ Under vacuum, the azulene can easily sublime and contaminate the gas lines and vacuum pump. of course, this makes it easy to clean azulene contaminated gas lines by pumping vacuum for a few hours.

The azulene concentration in the pressure cell and in cuvettes was approximately $6 \times 10^{-3} \mathrm{M}$, assuming a volume of about $2.2 \mathrm{~cm}^{3}$ for the low pressure cell and a volume of approximately $2 / 3$ that for the high pressure cell. This is a reasonable concentration for moderate signal sizes, with $90 \%$ of the excite wavelength absorbed. Although dimers of azulene 
have never been seen in supersonic jet expansions, ${ }^{12}$ in nonpolar liquids there may be the possibility of dimers forming. ${ }^{13}$ Data collected with solutions three times higher and lower in concentration showed no nonlinear effects, which indicates that dimerization and solvent complexation are not causing or affecting the reported signal.

\subsection{Excited state Dynamies studies}

Generally, the hot ground state azulene was probed to the red of the $s_{0}-s_{1}$ origin at $700 \mathrm{~nm}$. A majority of the data were taken at $740 \mathrm{~nm}$, very near the probe wavelength of other researchers." Some were also taken at $720 \mathrm{~nm}$ and $760 \mathrm{~nm}$, though the signal can no longer be detected any further to the red. A $620 \mathrm{~nm}$ short pass filter was placed in the excite during these experiments to remove nanosecond red fluorescence from the dye cell amplifiers. ${ }^{14}$

Attempts probing further to the blue, to examine the ground state bleach dynamics, were not successful. The bleach signal would help to confirm the vibrational cooling lifetimes that were measured since presumably the ground state bleach would decay away on the same time scale as the vibrational cooling of molecules as they return to equilibrium in $s_{0}$. Probing at $680 \mathrm{~nm}$ yielded a value for the decay very similar to that seen at $740 \mathrm{~nm}$. However, it is likely that a bleach signal is convoluted with the transient absorption at this wavelength, making it difficult to interpret this result. 
Probe studies within $90 \mathrm{~nm}$ either side of the excite wavelength were too difficult to pursue due to increased noise on the signal.

When the probe was tuned to $500 \mathrm{~nm}$, the lifetime of the $\mathrm{S}_{1}$ state was successfully measured. A pulse width limited transient absorption of approximately 2 psec was measured (see Figure 2.2) in accord with results obtained by Shank et al. 15 They determined the excited state lifetime to be 1.9 psec by monitoring fluorescence from $s_{2}$ as a function of time delay between the two photons used to reach the second excited state. They measured an identical time when they attenuated one of the beams to use as a probe of the ground state, similar to the arrangement used here, and attributed it to ground state recovery. However, it is more likely that they merely were probing the $s_{1}$ population and thus measuring the same phenomenon two different ways.

Azulene absorbs very strongly in the UV with the so-s2 band edge starting near $28,500 \mathrm{~cm}^{-1}(350 \mathrm{~nm})$ and peaking at $29,400 \mathrm{~cm}^{-1}(340 \mathrm{~nm})$ with an extinction coefficient of $5 \times 10^{4}$ $\mathrm{M}^{-1} \mathrm{Cm}^{-1} .^{8}$ Thus it is possible to excite azulene into the second excited state and examine the internal conversion to and subsequent vibrational cooling in the ground state. An attempt to do this by photoexcitation at $295 \mathrm{~nm}$ was not successful. The UV pulse was obtained by frequency doubling in a KDP crystal, telescopically focussing the $590 \mathrm{~nm}$ pulse by a factor of four into the crystal for $10 \cdots 15 \%$ conversion 
CHANGE IN ABSORBANCE $(\times 1 D D)$

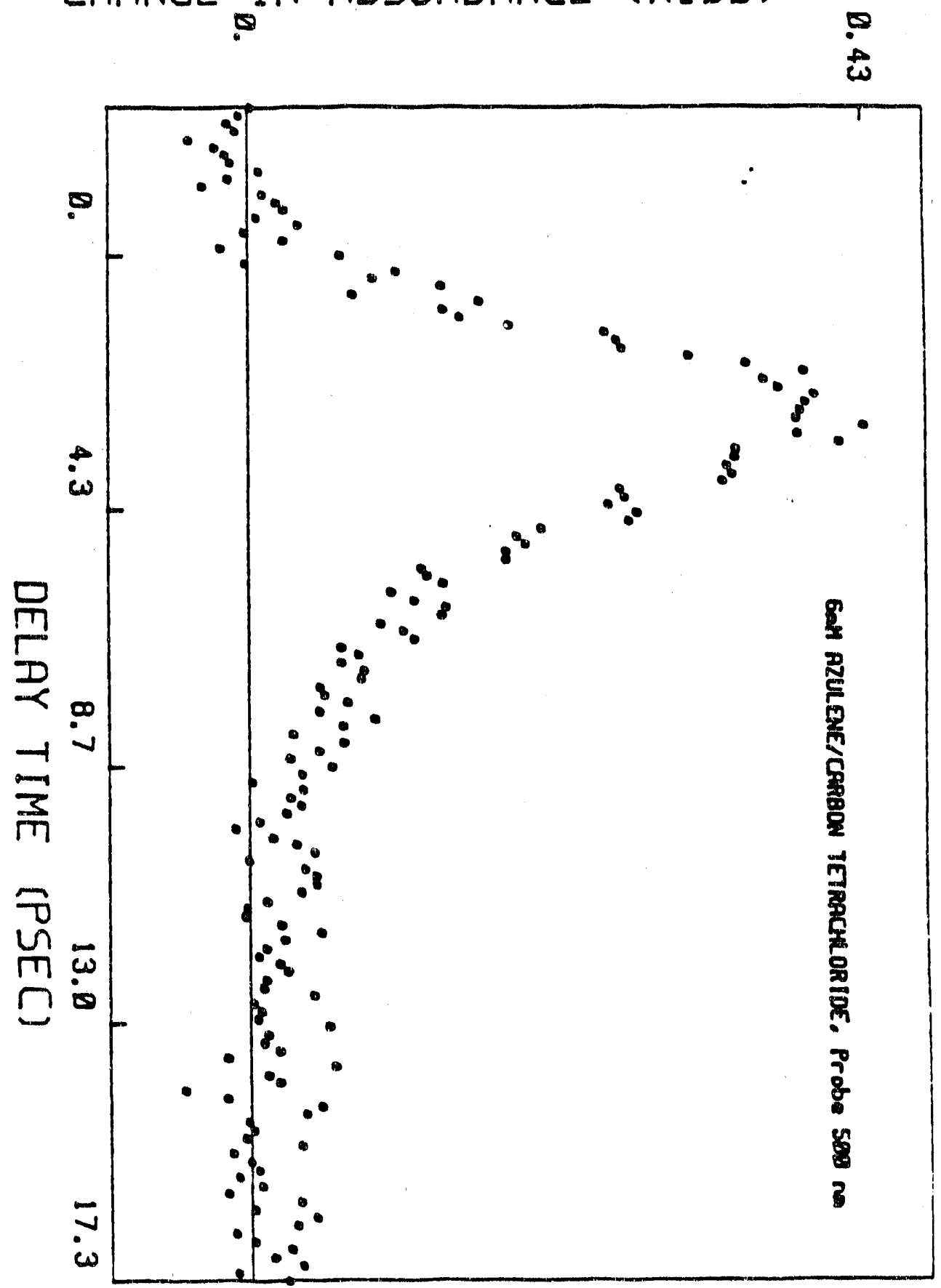

Figure 2.2 pulse width limited transient absorption signal, attributed to absorption from $S_{1}$ to $S_{2}$. Deconvolution of 1.5 ps laser pulse yields $S_{1}$ lifetime of 2.4 ps. 
efficiency. Although care was taken to purify solute and solvent and the UV excite was attenuated, photoproduct formation occurred almost instantly when the sample was illuminated. No signal due to fluorescence or absorption could be detected.

Because of this strong absorption in the UV, the dependence of the present data on the power of the laser was examined to determine whether multiphoton processes are responsible for the observed signal. spectroscopic grade solvents were consistently used to avoid interference due to impurities. Attenuating the excite caused a linear change in the signal size, indicating that the signals measured were not due to absorption of two $590 \mathrm{~nm}$ photons populating the second excited state. Experiments without the azulene in the cell showed no absorption changes due to dynamics in the solvent alone.

\subsection{Solvent Thermodynamic Information}

The experiments were performed over a range of liquid and fluid densities. This required a great deal of information about the phase diagrams of the gases used as solvents. ${ }^{16}$ The experimental thermodynamic conditions are shown in Table 2.1. The error in the gauges of the system are $2 \%$ of full scale which limits the accuracy of the pressure values listed in Table 2.1. These thermodynamic conditions are also indicated on a general Lennard-Jones phase diagram in 
Table 2.1 PVT thermodynamic information for solvent thermodynamic states used in experiments. See References 16, 17 and text.

\begin{tabular}{|l|l|l|l|l|l|}
\hline BOLVENT & $\rho^{*}$ & $\begin{array}{l}\rho \\
(\mathrm{g} / \mathrm{ml})\end{array}$ & $\mathrm{T}^{*}$ & $\begin{array}{l}\left.\mathrm{T}^{\circ} \mathrm{K}\right) \\
(\mathrm{Pe}\end{array}$ & $\begin{array}{l}\text { PRESSURE } \\
(\mathrm{psi})\end{array}$ \\
\hline $\mathrm{Xe}$ & 0.57 & 1.8 & 1.26 & 280 & 800 \\
\hline $\mathrm{Xe}$ & 0.88 & 2.8 & 1.26 & 280 & 14,500 \\
\hline $\mathrm{Xe}$ & 0.95 & 3.0 & 1.14 & 253 & 18,400 \\
\hline $\mathrm{Xe}$ & 0.95 & 3.0 & 1.26 & 280 & 24,000 \\
\hline $\mathrm{Xe}$ & 0.95 & 3.0 & 1.37 & 298 & 26,200 \\
\hline $\mathrm{Xe}$ & 0.95 & 3.0 & 1.46 & 323 & 30,200 \\
\hline $\mathrm{Kr}$ & 0.57 & 1.6 & 1.70 & 280 & 6,200 \\
\hline $\mathrm{Kr}$ & 0.82 & 2.3 & 1.70 & 280 & 25,500 \\
\hline $\mathrm{CClF} 3$ & 0.63 & 0.89 & 1.46 & 298 & 600 \\
\hline $\mathrm{CClF}$ & 0.63 & 0.89 & 1.48 & 303 & 710 \\
\hline $\mathrm{CClF} 3$ & 1.0 & 1.5 & 1.48 & 303 & 9,000 \\
\hline
\end{tabular}

reduced density $\rho^{*}=\rho \sigma^{3}$

reduced temperature $T^{*}=k_{B} T / \epsilon$ 


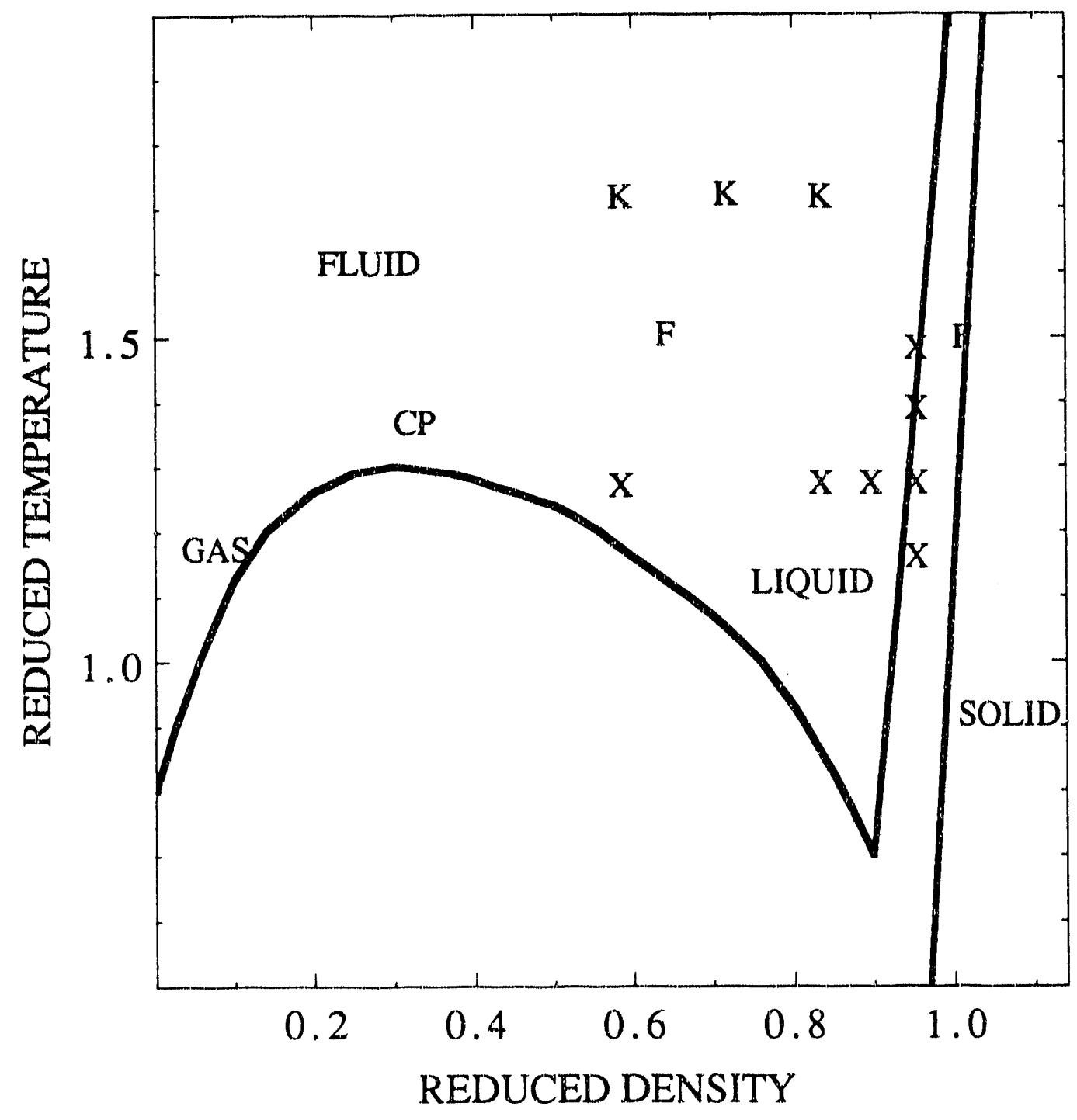

Figure 2.3 General Lennard-Jones phase diagram, indicating the temperature and densities of solvents used. $\mathrm{X}=\mathrm{Xe}, \mathrm{K}=$ $\mathrm{Kr}, \mathrm{F}=\mathrm{CClF}_{3}$. 
Figure 2.3. Not all of the solvents are good Lennard-Jones fluids, which is why some of the data points extend out of the Lennard-Jones liquid region of the phase diagram. However this is a good method for comparing the different samples. The units in the Lennard-Jones phase diagrain are reduced temperature, $T^{*}$, and reduced density, $\rho^{*}$. These are defined as $T^{*}=k_{B} T / \epsilon$ and $\rho^{*}=\rho / \sigma^{3}$, where $\epsilon$ is the Lennard-Jones potential well depth and $\sigma$ is the Lennard-Jones radius. These units describe the temperature of the system relative to the potential well and the number density of the atoms relative to their size. Table 2.2 lists the value of $\epsilon$ and $\sigma$ used for the three gases and azulene. 17,18 
Table 2.2 Lennard-Jones diameter ( $\sigma$ ) and well depth ( $\epsilon$ ) for solvent gases used in this work. See Reference 17 and 18 .

\begin{tabular}{|l|l|l|}
\hline Solvent & $\epsilon / K_{B}\left({ }^{\circ} \mathrm{K}\right)$ & $\sigma(\AA)$ \\
\hline \hline Xenon & 222 & 4.07 \\
\hline Krypton & 165 & 3.68 \\
\hline Freon-13 $\left(C C l F_{3}\right)$ & 204 & 4.95 \\
\hline Azulene & 523 & 6.61 \\
\hline
\end{tabular}




\section{References for Chapter 2}

1. M. Berg, A.L. Harris, J.K. Brown, and C.B. Harris, Opt. Lett. 9, $50(1984)$.

2. H.E. Lessing and A. von Jena, Chem. Phys. Lett. 12,213 $(1976)$.

3. Bruce $J$. Berne and Robert Pecora, Dynamic Light Scattering, (Wiley, New York, 1976), pp. 145-150.

4. W. Wild, A. Seilmeier, N.H. Gottfried, and W. Kaiser, Chem. Phys. Lett. 119, 259 (1985).

5. W.F. Sherman and A.A. Stadtmuller, Experimental Techniques in High Pressure Research, (Wiley, Chichester, 1987), pp. 365-369.

6. Sapphire has an index of refraction of approximately 1.71.8 throughout the visible spectral region. See John H. Moore, Christopher C. Davis, and Michael A. Coplan, Building Scientific Apparatus, 2nd ed. (Addison-Wesley, Reading, MA, 1989), p. 180 .

7. Mark E. Paige, Ph.D. thesis, Density Effects on the Molecular Dynamics of the $I_{2}$ Geminate Recombination Reaction in Liquid Xe, University of California - Berkeley, Berkeley, CA 1990; M.E. Paige and C.B. Harris, Chem. Phys. 149, 37 (1990).

8. Dieter Klemp and Bernhard Nickel, Chem. Phys. Lett. 130, 493 (1986).

9. H. Hoyer and W. Peperle, Z. Elektrochem., 62, 61 (1958).

10. Michel J. Rossi, Jack R. Pladziewicz, and John R. Barker, J. Chem. Phys. 78, 6695 (1983).

11. A. Bauder and Hs.H. Günthard, Helv. Chim. Acta, 196, 1698 (1962).

12. Aviv Amirav and Joshua Jortner, J. Chem. Phys. 81, 4200 (1984).

13. Jerry F. Deye, T. A. Berger, and Albert G. Anderson, Anal. Chem. 62, 615 (1990).

14. Alex L. Harris, Ph.D. thesis, Picosecond spectroscopy of Chemical Reactions in Liquids, University of California Berkeley, Berkeley, CA 1985. 
15. C.V. Shank, E.P. Ippen, O. Teschke, and R.I. Fork, Chem. Phys. Lett, 57, 433 (1978).

16. For thermodynamic information on $\mathrm{Xe}$ and $\mathrm{Kr}: \mathrm{D}$. Vidal, $\mathrm{L}$. Guengant and J. Vermesse, Physica 116A, 227 (1982); Jan Jüza and Oldłich sifner, Acta. Technica CSAV 1, 1 (1976); W.B. streett, L.S. Sagan and I.A.K. Staveley, J. Chem. Thermodynamics 5, 633 (1973): Felix Theeuwes and Richard Bearman, J. Chem. Thermodynamics 2, 501 (1970); A. Michels, T. Wassenaar and P. Lourwerse, Physica 20, 99 (1954); For thermodynamic information on $\mathrm{CClF}_{3}$ : B. Platzer, A. Polt, and G. Maurer, Thermophysical Properties of Refrigerants, (Springer-Verlag, Berlin, 1990), pp. 77-99; K.R. Harris, Physica 93A, 593 (1978); L.F. Albright and J.J. Martin, Industrial and Engineering Chemistry, 44, 188 (1952).

17. For $\mathrm{Kr}$ and Xe parameters: D. Vidal, I. Guengant, and J. Vermesse, Physica 116. 227 (1982); A.J. Ledbetter and H.E. Thomas, Trans. Faraday Soc. 61, 1.0 (1965); For $\mathrm{CClF}_{3}$ parameters: K.R. Harris, Physica 116A, 593 (1978); Mitsuo Takahashi, Shinji Takahashi, and Hiroji Iwasaki, J. Chem. Eng. Data 30, 10 (1985).

18. For azulene Lennard-Jones parameters see H. Hippler, B. otto, and J. Troe, Ber. Bunsenges. Phys. Chem. 93, 428 (1989). 
Chapter 3: RESULTS

\subsection{General Information}

\section{1.a Data Fitting Process}

Theory ${ }^{1}$ and experiments in the gas phase ${ }^{2}$ have shown that the vibrational cooling of azulene occurs as an exponential function of time. Therefore, all transient absorptions were fit with following equation:

$$
f(t)=c *\left(1-e^{-t / a}\right) * e^{-t / b}
$$

where $a$ is the rise time of the probe absorbance, $b$ is the decay of the probe absorbance and $c$ is the amplitude of the absorbance. This equation sufficiently accounts for the photophysical reactions occurring in the azulene.

Physically, the rise time corresponds to the time required for the azulene in $S_{1}$ to internally convert and enter the ground state. The rise time is essentially pulse width limited to a few picoseconds in all solutions, at all densities. A dramatic density dependence to the internal conversion time is not observed due to the small geometric change that occurs between ground and excited state azulene. ${ }^{3}$ The rapid rise time observed in the experiment is consistent with measurements by others of an internal conversion time of less than 2 psec. ${ }^{4}$

The decay of the transient absorption occurs as the hot ground state molecules lose their energy to the solvent. The 
molecules cool and return to equilibrium, at which point the probe wavelength is no longer absorbed. This decay time directly corresponds to the vibrational cooling time of azulene in the ground state. It is the subsequent change in this lifetime with changing solvent environment that is being experimentally determined in this work.

The signal sizes are typically quite small, about 0.1 $0.2 \%$ change in absorbance. This is due to the small absorption coefficient of azulene at $590 \mathrm{~nm}$, about 300 $M^{-1} \mathrm{Cm}^{-1}, 5$ and the even smaller absorption coefficient of the hot molecules at the probe wavelength, about $90 \mathrm{M}^{-1} \mathrm{~cm}^{-1} .^{6}$ Approximately 600-800 laser shots per data point were averaged over about four hours of collection time to obtain sufficiently good signal to noise to see these small transient signals.

The best fit of the data to the equation above was found by least squares minimization using an interactive graphic fitting routine with the exponential fitting equation above. An alternative method to obtain the best fit to the data used a linear regression to fit a straight line to the natural log of the data. This yielded a vibrational cooling time very close to that obtained from the interactive routine.

Once the best fit was found, the uncertainty of this fit needed to be determined. This was done by minimizing the chisquare parameter after investigating a Gaussian range of values centered about the best fit value. A rough, 
alternative way to check the error in the best values for the fit can be obtained by using the residuals (the difference between the data and the best fit to the data) obtained from the interactive fitting routine. The standard deviation of the residuals from the fit can be routinely calculated. The decay parameter of the fit, $b$, is adjusted so that it will overlap the data within one standard deviation of the residuals. In this way, one obtains two fitting curves which overlap the extremes of the data. The values for $b$ at each extreme are then considered to be $b \pm 1 \sigma$, one standard deviation unit.?

A more complicated way to obtain a value for the standard deviation in the decay, can be found in other mathematical texts. ${ }^{8}$ However, this routine requires that the data and the fit be in linear format. The natural logarithm of the data puts it into linear form, since the behavior measured is a single exponential. Unfortunately, in taking the natural logarithm of the data, the noise distribution is no longer Gaussian, which is the assumption made in least squares minimization. Because the natural logarithm increases the noise with time, usually two values for the standard deviation of the fit from the line of the data were used.

Generally, it appears that these three methods of determining the value for the error in the decay time, $b$, support one another. The values obtained for the range in $b$ from the interactive fitting routine generally correspond to 
approximately two times the values for the standard deviation in $b$ obtained from the two other fitting methods. The values of the decay times listed for each experimental condition are always shown throughout this thesis with the average value b $\pm 2 \sigma$ (two standard deviations). This gives an upper limit on the value of the decay to within 978 confidence i.e. 978 of the time the measured value of the decay will be within the stated region.

\section{1.b Stimulated Emission Detection}

In many of the decays, a pulse width limited bleach spike was seen at $t_{0}$. A typical example of this is shown in Figure 3.1. It occurred frequently in the chloromethanes, methanol, and hexane solutions, but rarely in compressed gas solvents. It did not occur with solvent alone and therefore was not a coherence artifact of the solvent. It would often appear, but was not always present in all scans. The size of the spike was correlated with the concentration of the azulene in solution. It was generally similar in size to the transient absorption signal and had a linear dependence on the excite intensity.

This spike could be a measurement of the bleach at the probe absorbance wavelength that occurs upon excitation of the ground state molecules to the first excited state. In the process of excitation to $s_{1}$, population is removed from $S_{0}$. If the probe pulse is absorbed by the ground state molecules, 
CHANGE IN ABSORBANCE $(\times 1 D D)$

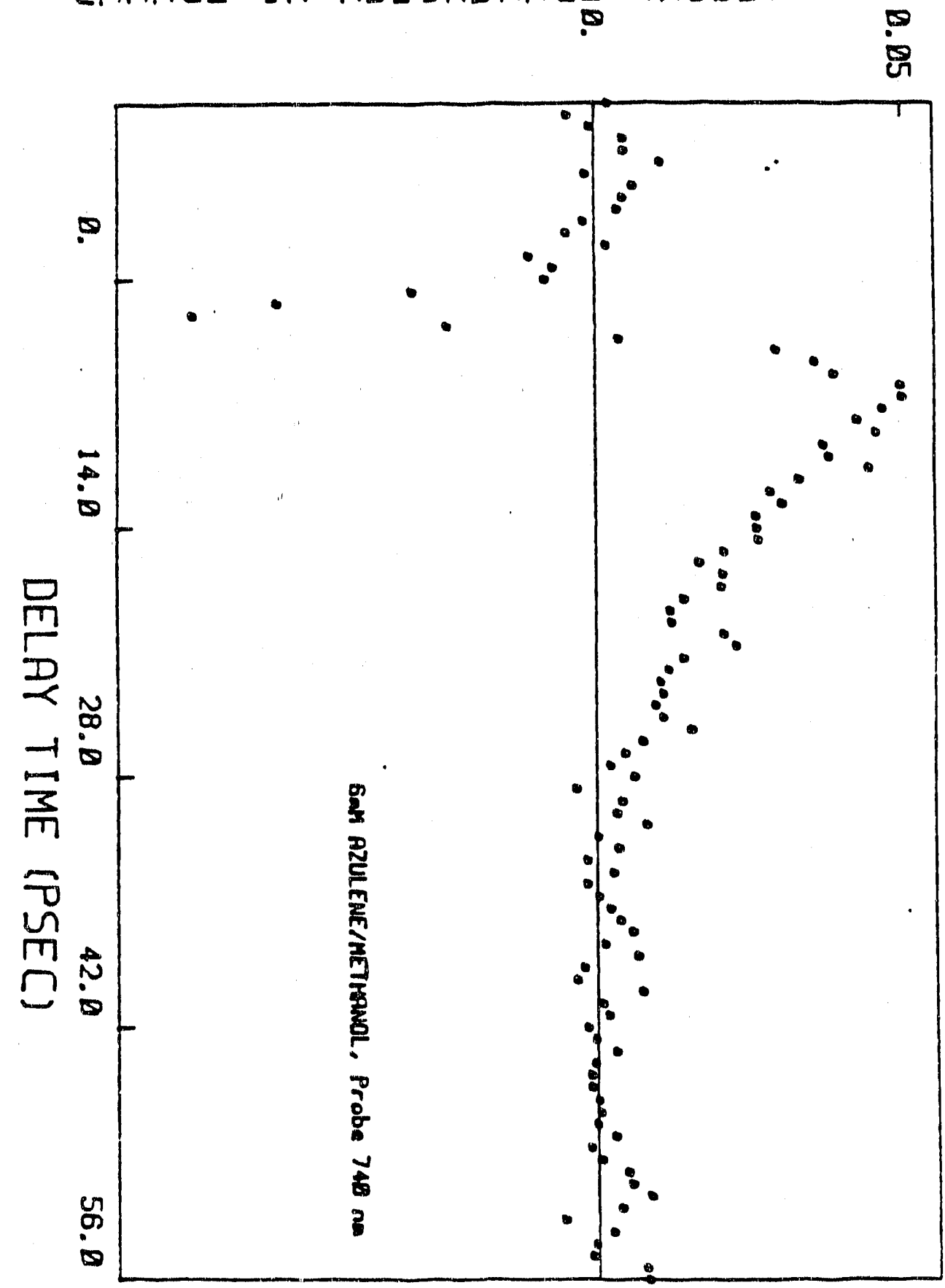

Figure 3.1 Transient absorption signal showing early time spike, attributed to stimulated emission from $s_{1}$. 
it will suddenly decrease in absorption, i.e. bleach, due to the loss of ground state population. This is a possible explanation for this effect as the probe wavelengths $760-720$ $\mathrm{nm}$ are minimally absorbed by the ground state azulene, $(\epsilon=$ 10-100 $\left.\mathrm{M}^{-1} \mathrm{~cm}^{-1}\right)^{6}$ and there is a trend with wavelength, the stronger absorbing $720 \mathrm{~nm}$ probe showing larger spikes than the $760 \mathrm{~nm}$ probe. However, this does not explain why it would be seen in only certain solvents and only intermittently.

More likely, this signal is stimulated emission from the first excited state which would only occur when the pump and probe beams are overlapped at zero time. The emission from the $s_{1}$ state occurs in the $700-800 \mathrm{~nm}$ region. The fluorescence from the first excited state is weak due to the rapid internal conversion time however a strong laser pulse could induce a small amount of the excited state population to emit. In fact, the intense picosecond ruby laser pulse was used to initially discover the fluorescence from $s_{1} \cdot{ }^{9}$ In addition, the linear power dependence supports this explanation. Since a certain population in $S_{1}$ would be required before stimulated emission can occur, this would also explain the sporadic appearance of this feature.

It is unlikely that this signal could be due to higher states. The probe wavelengths were chosen to minimize the possibility of the second excited state interfering with the photophysics. One pump and one probe photon together are $30,100 \mathrm{~cm}^{-1}$ energy, just barely enough energy to reach $s_{2}$. 
Shifts in energy levels in different solvents are possible, which might lead to a solvent dependence. However, the population of excited molecules in S1 upon excitation is about $1.3 \times 10^{15}$, approximately $90 \%$ of the $1.5 \times 10^{15}$ photons in the excite pulse. The absorption coefficient for the transition from $S_{1}$ to $S_{2}$ is weak, approximately $300 \mathrm{M}^{-1} \mathrm{~cm}^{-1}{ }^{10}$ Therefore only a small population of molecules could reach the second excited state due to pump-probe two photon absorption and can then fluoresce in the UV.

Since this effect appears to be due to stimulated emission from $s_{1}$, it does not interfere with other aspects of the data. Therefore, other than Figure 3.1 which is shown to demonstrate this effect, all other scans have had this effect removed from the data to facilitate presentation and comparison among scans.

\subsection{Solvent Trends}

\section{2.a Probe Wavelength Dependence}

As has been seen in previous studies of vibrational relaxation, 11,12 the vibrational relaxation rate measured is dependent upon the choice of probe wavelength (see Figure 3.2 and Table 3.1). This is understandable in that different probe wavelengths interrogate different regions of the azulene potential surface. The different populations will relax at different rates depending upon the coupling to the solvent. 


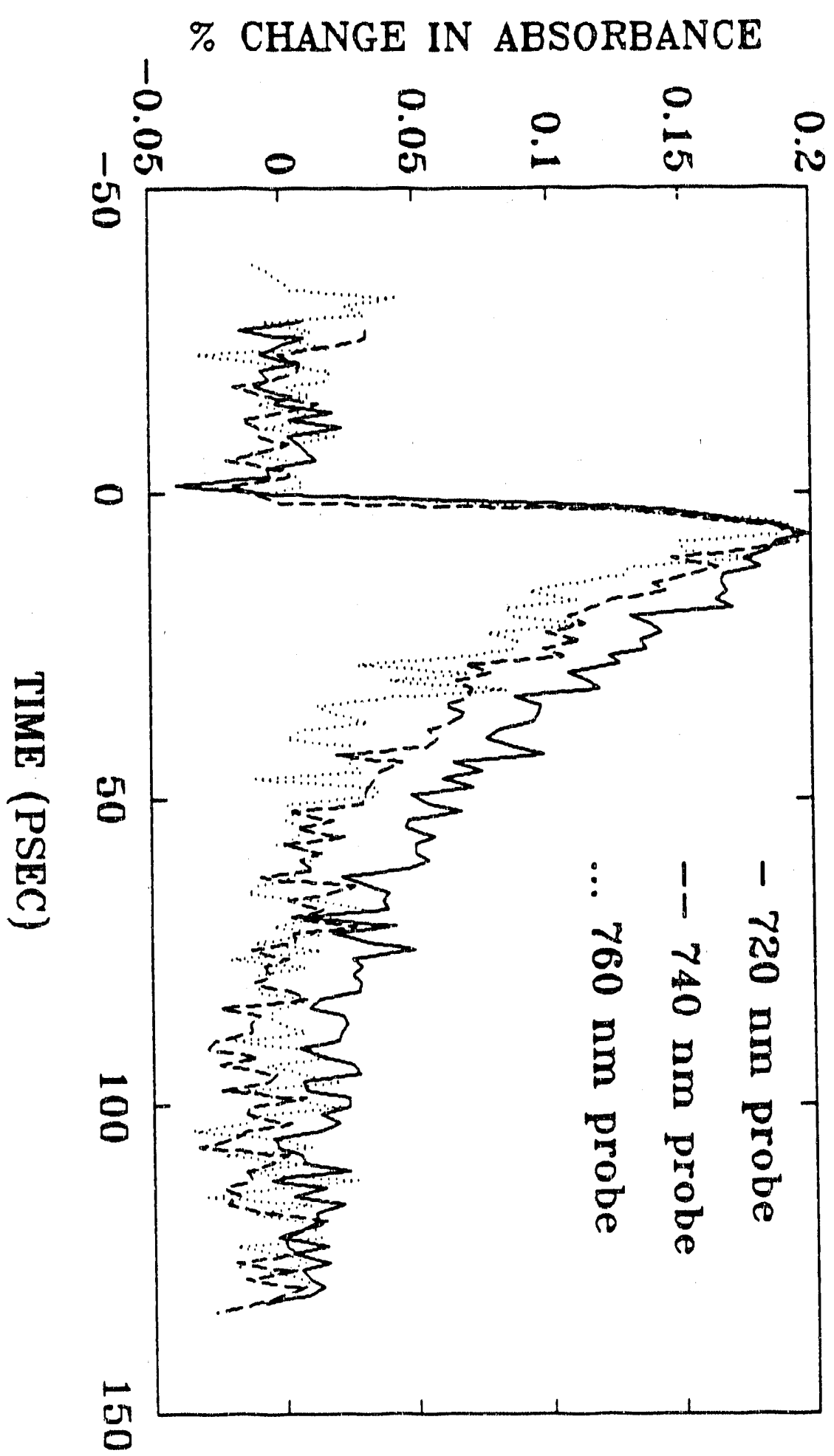

Pigure 3.2 Effect of probe wavelength on vibrational cooling rates in $\mathrm{CCl}_{4},-=720 \mathrm{~nm},--=740 \mathrm{~nm}, \ldots=760 \mathrm{~nm}$. Signal size of $720 \mathrm{~nm}$ scan decreased by 0.7 to allow comparison. 
Table 3.1 Vibrational relaxation times as a function of probe wavelength.

\begin{tabular}{|l|l|l|l|l|}
\hline SOLVENT & $\rho^{*}$ & $\begin{array}{l}\mathrm{T}(\mathrm{K}) \\
\pm \mathrm{I}^{\circ}\end{array}$ & $\begin{array}{l}\text { COOLING } \\
\text { TIME (psec) }\end{array}$ & $\begin{array}{l}\text { WAVELENGTH } \\
(\mathrm{nm})\end{array}$ \\
\hline $\mathrm{CCl}_{4}$ & 1.1 & 298 & $34 \pm 5$ & 720 \\
\hline $\mathrm{CCl}_{4}$ & 1.1 & 298 & $22 \pm 4$ & 740 \\
\hline $\mathrm{CCl}_{4}$ & 1.1 & 298 & $17 \pm 6$ & 760 \\
\hline $\mathrm{Xe}$ & 0.57 & 280 & $170 \pm 42$ & 740 \\
\hline $\mathrm{Xe}$ & 0.57 & 280 & $160 \pm 56$ & 760 \\
\hline $\mathrm{Xe}$ & 0.82 & 280 & $175 \pm 20$ & 740 \\
\hline $\mathrm{Xe}$ & 0.82 & 280 & $140 \pm 24$ & 760 \\
\hline $\mathrm{Xe}$ & 0.95 & 280 & $137 \pm 16$ & 740 \\
\hline $\mathrm{Xe}$ & 0.95 & 280 & $100 \pm 22$ & 760 \\
\hline
\end{tabular}


In azulene, the trend is that the rate of vibrational relaxation is more rapid when probed at longer wavelengths. Wavelengths longer than $700 \mathrm{~nm}$, the $(0,0)$ origin, probe vibrational hot bands. The longer probe wavelengths measure the dynamics of higher frequency hot bands. These higher energy modes relax into lower energy modes, which are probed by shorter probe wavelengths. Therefore the low energy, low frequency bands take longer to relax and the shorter probe wavelengths measure a longer cooling time. This shift in measured relaxation rates with wavelength was postulated by others earlier. 6 Wavelength dependence is a signature of the process of vibrational relaxation and confirms the fact that what we are studying is indeed vibrational cooling.

It should be noted that we did not find a wavelength dependence in the pulse width limited signal rise time. Any apparent change in the rise time seen in Figure 3.2 is due to a difference in the time step between data points used at different probe wavelengths. This wavelength independence is presumably because IVR within the azulene is fast enough that upon internal conversion into the ground state, the energy has been distributed at once into many different vibrational modes. ${ }^{13}$

\section{2.b Solvent Mass Dependence}

The use of krypton and xenon as solvents allows a unique comparison of two similar solvents that differ most notably in 


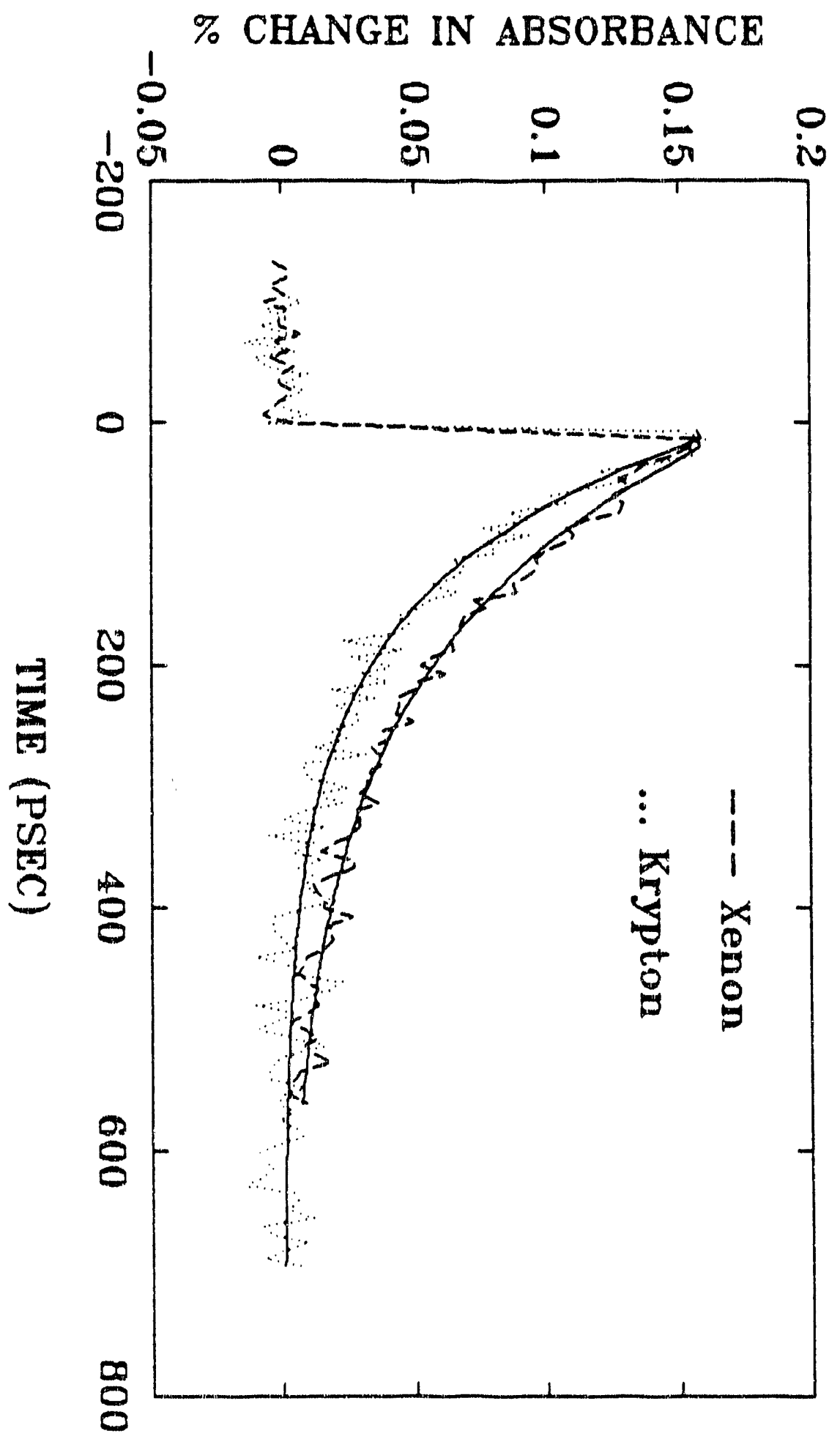

Pigure 3.3 Effect of solvent mass on vibrational relaxation rate. Solia lines are best exponential fit. Krypton signal size increased by 1.3 to allow comparison. 
Table 3.2 Vibrational cooling times as a function of solvent mass.

\begin{tabular}{|l|l|l|l|l|}
\hline SOLVENT & $\rho^{*}$ & $\begin{array}{l}\text { T }(\mathrm{K}) \\
\pm 1^{\circ}\end{array}$ & $\begin{array}{l}\text { COOLING TIME } \\
(\mathrm{psec})\end{array}$ & $\begin{array}{l}\text { WAVELENGTH } \\
(\mathrm{nm})\end{array}$ \\
\hline $\mathrm{Xe}$ & 0.57 & 280 & $170 \pm 42$ & 740 \\
\hline $\mathrm{Kr}$ & 0.57 & 280 & $133 \pm 32$ & 740 \\
\hline $\mathrm{Xe}$ & 0.82 & 280 & $175 \pm 20$ & 740 \\
\hline $\mathrm{Kr}$ & 0.82 & 280 & $118 \pm 24$ & 740 \\
\hline
\end{tabular}


their mass. Their potentials are somewhat different also. our results show that the cooling rate in krypton is only slightly faster than that in xenon at the same reduced density. A comparison of vibrational cooling lifetimes in xenon and krypton at the same reduced density (i.e. the same number density, not the same mass density) are shown in Figure 3.3 and Table 3.2. A plot of the lower density decays shows a similar result however with larger signal to noise. It should be reiterated that this difference in the dynamics in the two atomic solvents is not due to the heavy atom effect, as mentioned in section 1.1.c.

\subsection{Temperature Dependence}

The process of vibrational cooling was studied over the range of temperatures $253 \mathrm{~K}$ - $323 \mathrm{~K}$. Considering that the azulene molecule reaches an internal vibrational temperature of $1100 \mathrm{~K}$ as soon as internal conversion occurs, the experimental temperature range is only a $6 \%$ change. Temperature independence over this range is not surprising. Figure 3.4 demonstrates this phenomenon at the highest and lowest temperatures. Table 3.3 lists the vibrational cooling times.

\section{2.d Solvent Density Dependence}

The high pressure optical cell used in this study provides us with a unique opportunity to cleanly separate the 
Table 3.3 Vibrational relaxation times as a function of solvent temperature.

\begin{tabular}{|l|l|l|l|l|}
\hline SOLVENT & $\rho^{*}$ & $\begin{array}{l}\text { T (K) } \\
\pm 1^{\circ}\end{array}$ & $\begin{array}{l}\text { COOLING } \\
\text { TIME (psec) }\end{array}$ & $\begin{array}{l}\text { WAVELENGTH } \\
(\mathrm{nm})\end{array}$ \\
\hline $\mathrm{Xe}$ & 0.95 & 323 & $130 \pm 20$ & 740 \\
\hline $\mathrm{Xe}$ & 0.95 & 298 & $128 \pm 24$ & 740 \\
\hline $\mathrm{Xe}$ & 0.95 & 280 & $137 \pm 16$ & 740 \\
\hline $\mathrm{Xe}$ & 0.95 & 253 & $123 \pm 30$ & 740 \\
\hline
\end{tabular}




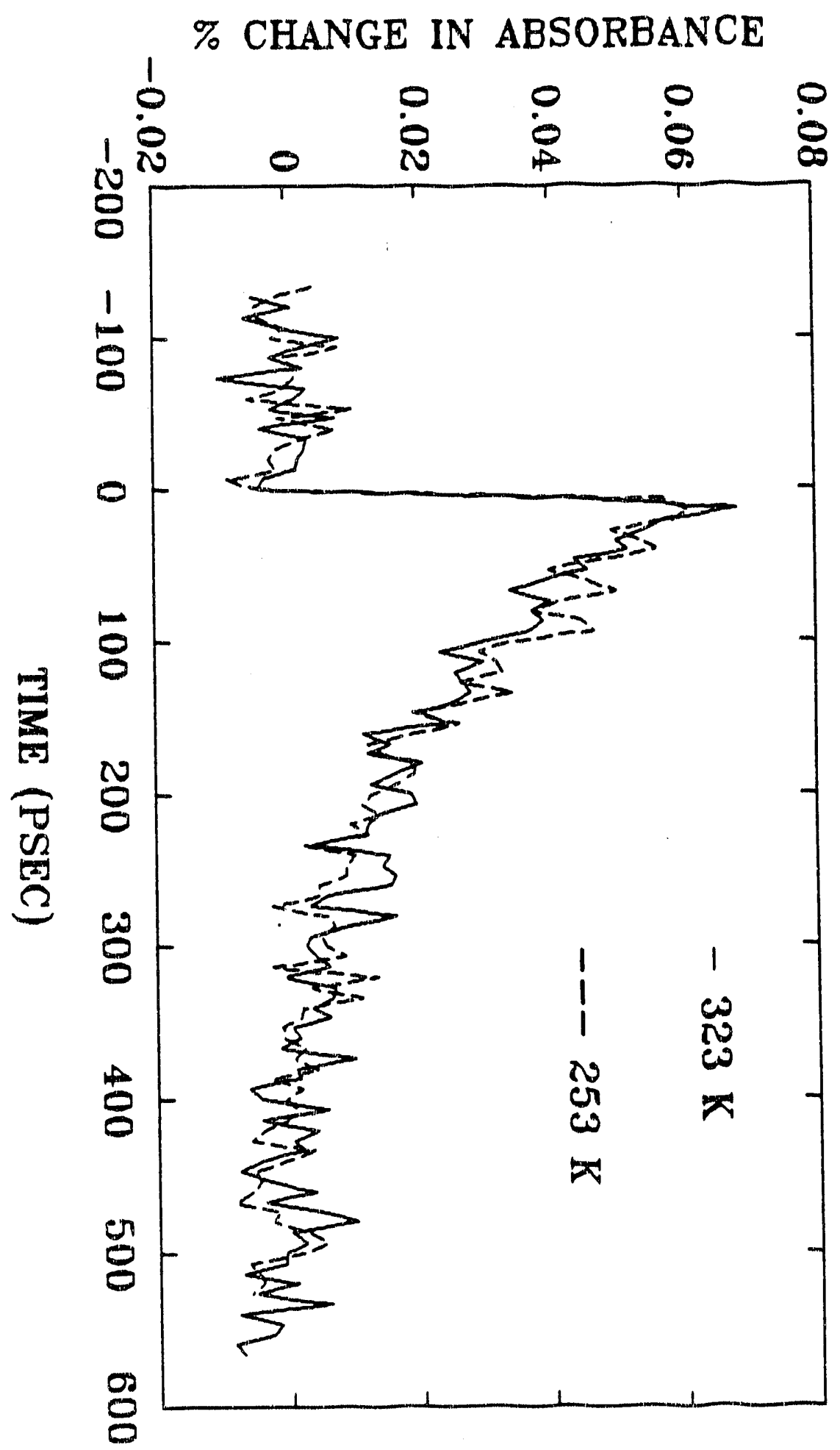

Pigure 3.4 Effect of solvent temperature on vibrational cooling rates. $253 \mathrm{~K}$ signal size increased by 1.25 to allow comparison. 
effect of solvent density on vibrational relaxation. The temperature of the system remains constant while the pressure is changed to increase or decrease the density. Unlike the gas phase, liquids require a large pressure differential in order to achieve a small change in solvent density. As a rule of thumb, one estimates that a $15,000 \mathrm{psi}(1000 \mathrm{~atm})$ change in pressure is required to produce a $1.0 \%$ change in the solvent density. ${ }^{14}$ The wide range of pressures accessed by the pressure system used here permits us to investigate dynamics throughout the entire liquid density range.

The results of changing solvent density on the vibrational cooling time indicates that there is a surprisingly small effect. The range of reduced density in xenon $\rho^{*}=0.57$ to $\rho^{*}=0.95$ affects the cooling rate by only $20 \%$ (see Figure 3.5 and Table 3.4 ). This result is surprising because the liquid environment in the two densities are dramatically different. The lowest density xenon solution has an open liquid structure and an occupied volume of 0.32 . The highest density solution is very nearly a solid with almost twice the occupied volume of the low density solution. one might expect that this environmental change would impact energy transfer dynamics. A similar density effect is mirrored in krypton (Figure 3.6) over a smaller density range and, to a lesser extent, in $\mathrm{CClF}_{3}$ (Figure 3.7).

In general, increasing the solvent density will increase the number of collisions between solute and solvent. One 


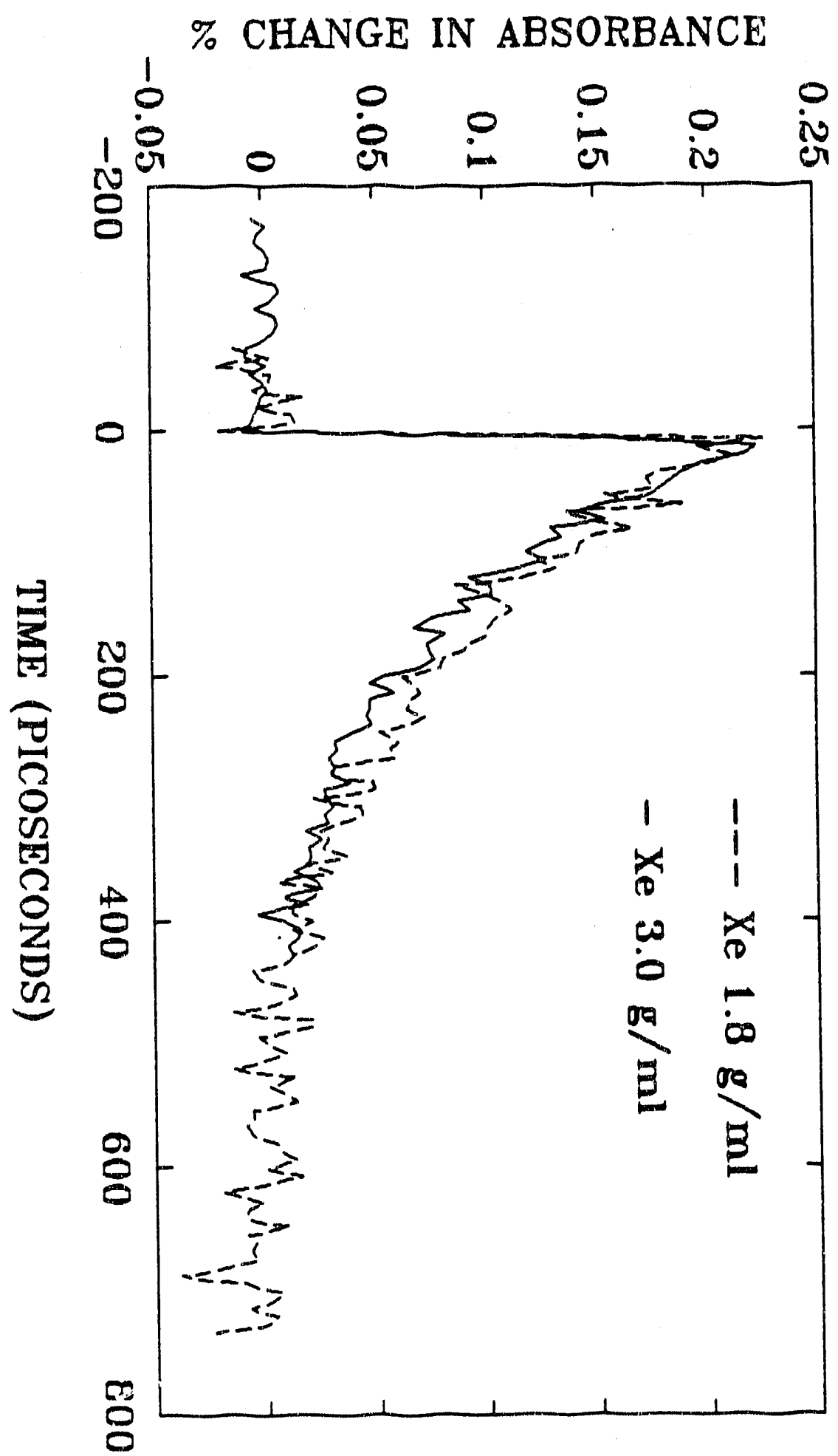

Figur 3.5 Effect of solvent density on vibrational cooling rate at probe wavelength $740 \mathrm{~nm}$ in xenon at $280 \mathrm{~K}$. High density signal increased by 1.45 to allow comparison. 
Table 3.4 Vibrational relaxation times as a function of solvent density.

\begin{tabular}{|l|l|l|l|l|}
\hline SOLVEN'T & $\rho^{*}$ & $\begin{array}{l}\mathrm{T}(\mathrm{K}) \\
\pm 1^{\circ}\end{array}$ & $\begin{array}{l}\text { COOLING } \\
\text { TIME (psec) }\end{array}$ & $\begin{array}{l}\text { WAVELENGTH } \\
(\mathrm{nm})\end{array}$ \\
\hline $\mathrm{Xe}$ & 0.57 & 280 & $170 \pm 42$ & 740 \\
\hline $\mathrm{Xe}$ & 0.82 & 280 & $175 \pm 20$ & 740 \\
\hline $\mathrm{Xe}$ & 0.88 & 280 & $150 \pm 30$ & 740 \\
\hline $\mathrm{Xe}$ & 0.95 & 280 & $137 \pm 16$ & 740 \\
\hline $\mathrm{Xe}$ & 0.57 & 280 & $160 \pm 56$ & 760 \\
\hline $\mathrm{Xe}$ & 0.82 & 280 & $140 \pm 24$ & 760 \\
\hline $\mathrm{Xe}$ & 0.95 & 280 & $100 \pm 22$ & 760 \\
\hline $\mathrm{Kr}$ & 0.57 & 280 & $133 \pm 32$ & 740 \\
\hline $\mathrm{Kr}$ & 0.82 & 280 & $118 \pm 24$ & 740 \\
\hline $\mathrm{CClF}$ & 0.63 & 303 & $57 \pm 8$ & 740 \\
\hline $\mathrm{CClF}$ & 1.0 & 303 & $36 \pm 15$ & 740 \\
\hline
\end{tabular}




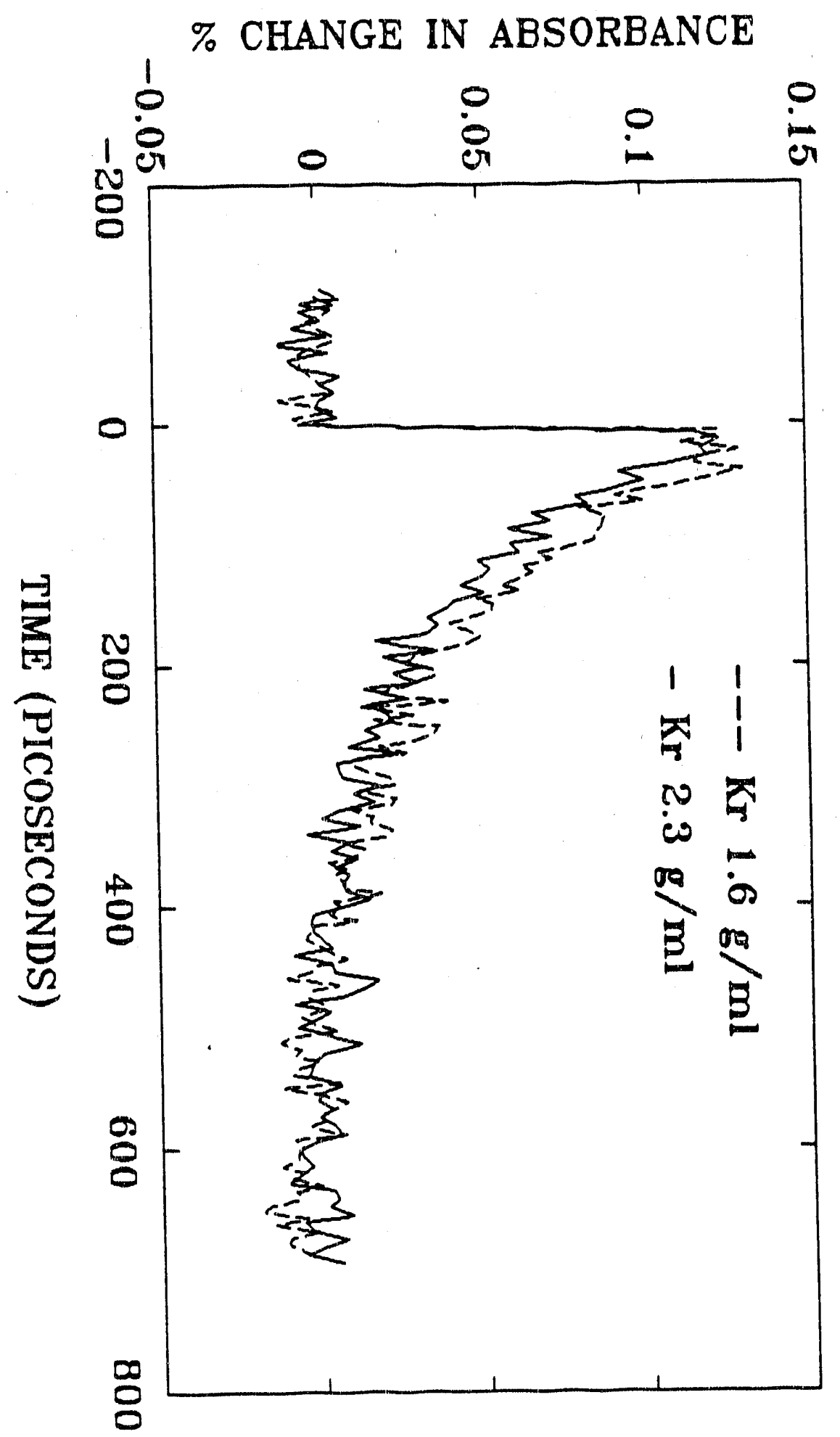

Pigure 3.6 Effect of solvent density on vibrational cooling rate at probe wavelength $740 \mathrm{~nm}$ in krypton at $280 \mathrm{~K}$. High density signal increased by 1.1 to allow comparison. 


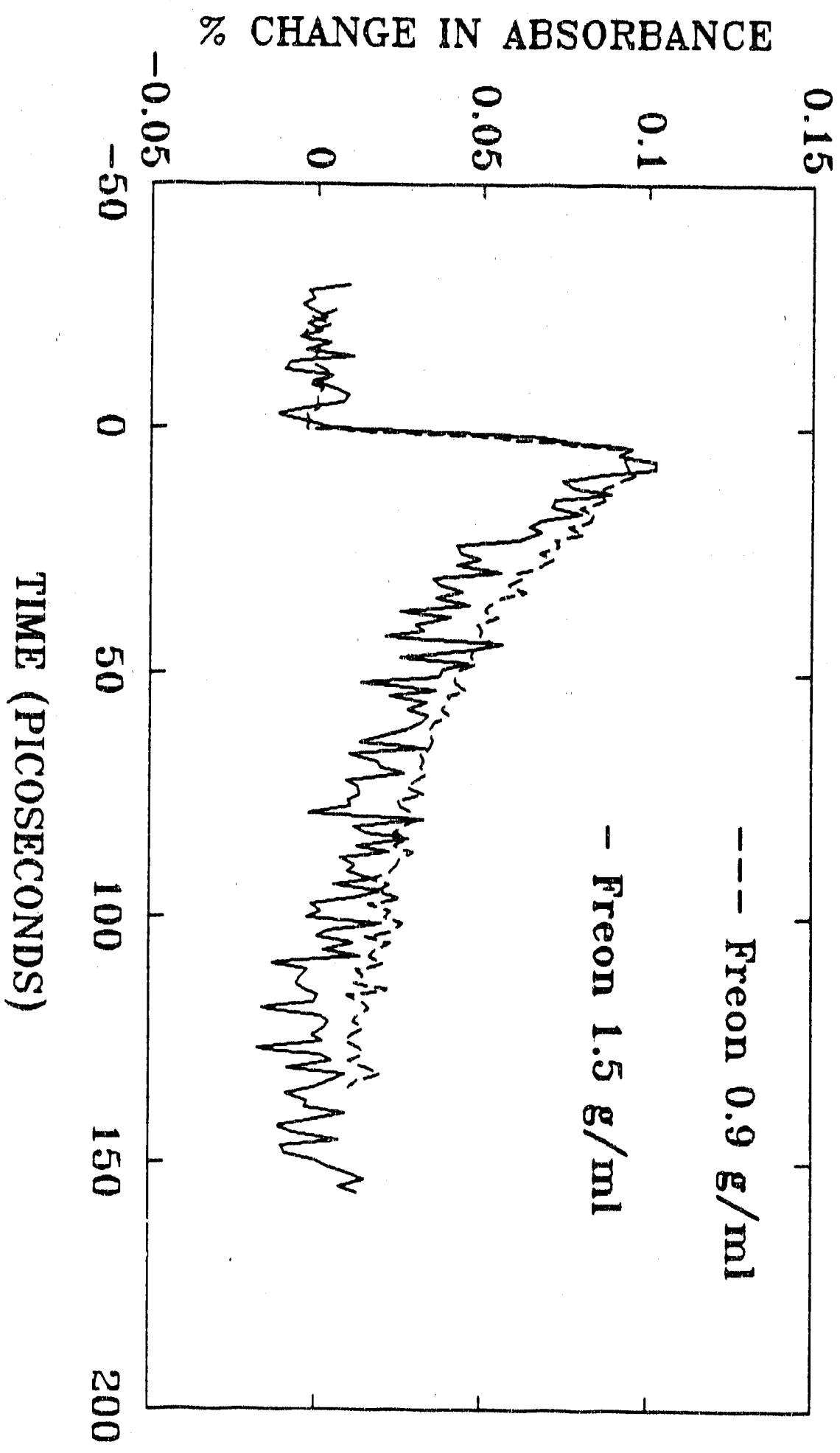

8igure 3.7 Effect of solvent density on vibrational cooling rate at probe wavelength $740 \mathrm{~nm}$ in $\mathrm{CClF}_{3}$ at $303 \mathrm{~K}$. High density signal increased by 2.75 to allow comparison. 
would therefore expect an increase in the energy transfer rate. In fact, previous studies ${ }^{15}$ showed a 3008 change in the vibrational relaxation rate of hot $I_{2}$ over the same Xe densities studied here. In addition, the relaxation times for geminately recombined iodine molecules was almost twenty times longer than the azulene relaxation times, presumably due to the lack of internal modes in the iodine to assist energy Ioss.

\section{2.e Solvent Isotope Effect}

The vibrational cooling process was studied in deuterium substituted chloromethane solvents (99.96\%D Aldrich). These results were compared to the results in corresponding protonated chloromethanes. The role of deuterium substitution in a solvent system is to illuminate the role of the solvent $\mathrm{C}-\mathrm{H}$ stretches in the process of vibrational relaxation. Deuterium substitution alters the frequency of the stretch and therefore any change in the relaxation time can be attributed to involvement with the $\mathrm{C}-\mathrm{H}$ mode. However, deuterium substitution can also alter other important modes in the molecule, bringing some into resonance that may not have been that important in the proto-solvent.

In this case, isotopic study could only be conducted in deuterated methylene chloride $\left(\mathrm{CD}_{2} \mathrm{Cl}_{2}\right)$. When the azulene study was carried out in deuterochloroform $\left(\mathrm{CDCl}_{3}\right)$, isotopic exchange between the azulene and the solvent occurred within 
the time scale of the experiment, affecting the experimental results. This is due to the fact that catalytic amounts of acid form in chloroform due to the stability of the $\mathrm{CCl}_{3}$. radical. ${ }^{16}$ It has been determined that azulene can lose up to two hydrogens from the 1,3 positions in the presence of acid, which facilitates the isotopic exchange process in solution. ${ }^{17}$ Methylene chloride forms much smaller amounts of acid and therefore isotopic exchange is not a significant problem.

The results with proto- and deutero- methylene chloride indicate that there is no change in the relaxation time of azulene in the two solvents. In $\mathrm{CH}_{2} \mathrm{Cl}_{2}$, the relaxation time is $19 \pm 3 \mathrm{psec}$ and in $\mathrm{CD}_{2} \mathrm{Cl}_{2}$, the relaxation time is $18 \pm 3$ psec. This indicates that the high frequency $\mathrm{C}-\mathrm{H}$ stretches are not involved in the relaxation process since lower frequency solvent modes are unaffected by deuterium substitution. ${ }^{18}$ This result is not surprising in light of the fact that the $\mathrm{C}-\mathrm{H}$ stretches are not involved with the $\mathrm{S}_{0}-\mathrm{S}_{1}$ transition or internal conversion (see section 1.1.f).

\section{2.f Solvent Dependence}

The vibrational cooling of ground state azulene has been siudied in a number of conventional and nonconventional solvents. This allows a wide range of solvents to be compared in terms of their ability to rapidly cool hot ground state azulene. The cooling rate in molecular solvents is 
approximately $20 \mathrm{psec}$, in reasonable agreement with other studies. 6 The rate in rare gas solvents is much slower, approximately $130 \mathrm{psec}$. This is presumably due to the lack of internal modes in these solvents, which limits the amount of energy transfer that can occur.

A number of different solvents were used iri this study including hexane, methanol and the chloromethanes and the relaxation times in each are 1 isted in Table 3.5. It is interesting that the three chloromethane solvents yield almost identical cooling times, when their masses, vibrational frequencies and rotational frequencies are so different. It is also interesting to see how rapidly the vibrational cooling of azulene can occur in hexane, a hydrocarbon that has many similar vibrational frequencies. ${ }^{19}$ Resonance between the two hydrocarbons, azulene and hexane, may facilitate $V-V$ energy transfer and speed relaxation. Because of the improved time resolution and lower excitation energy, the values for the cooling time that we have obtained here are shorter and more accurate than the values obtained by others. ${ }^{20}$ However similar trends in the cooling times in different solvents are seen in both works. 
Table 3.5 Vibrational relaxation rates in various solvents.

\begin{tabular}{|l|l|l|l|l|}
\hline SOLVENT & $p^{*}$ & $\begin{array}{l}\text { T (K) } \\
\pm 1\end{array}$ & $\begin{array}{l}\text { COOLING } \\
\text { TIME (psec) }\end{array}$ & $\begin{array}{l}\text { WAVELENGTH } \\
\text { (nm) }\end{array}$ \\
\hline $\mathrm{CCl}_{4}$ & 1.1 & 298 & $22 \pm 4$ & 740 \\
\hline $\mathrm{CHCl}_{3}$ & 1.0 & 298 & $23 \pm 3$ & 740 \\
\hline $\mathrm{CH}_{2} \mathrm{Cl}_{2}$ & 0.96 & 298 & $19 \pm 3$ & 740 \\
\hline $\mathrm{CH}_{3} \mathrm{OH}$ & 0.73 & 298 & $10 \pm 3$ & 740 \\
\hline $\mathrm{C}_{6} \mathrm{H}_{14}$ & $n .2$. & 298 & $15 \pm 2$ & 740 \\
\hline $\mathrm{CClF}_{3}$ & 0.63 & 298 & $42 \pm 10$ & 740 \\
\hline $\mathrm{Kr}$ & 0.82 & 280 & $118 \pm 24$ & 740 \\
\hline $\mathrm{Xe}$ & 0.95 & 298 & $128 \pm 24$ & 740 \\
\hline
\end{tabular}

n.a. = not applicable 


\section{References for Chapter 3}

1. Wendell Forst, Chem. Phys. 147, 241 (1990).

2. Michel J. Rossi, Jack R. Pladziewicz, and John R. Barker, J. Chem. Phys. 78, 6695 (1983); H. Hippler, B. Otto, and J. Troe, Ber. Bunsenges. Phys. Chem. 93, 428 (1989).

3. Klaus Gustav and Michael Storch, Intl. J. Quant. Chem. 38,25 (1990).

4. E.P. Ippen, C.V. Shank, and R.L. Woerner, Chem. Phys. Lett. 46,20 (1977).

5. Dieter Klemp and Bernhard Nickel, Chem. Phys. Lett. 130, 493 (1986).

6. W. Wild, A. Seilmeier, N.H. Gottfried, and W. Kaiser, Chem. Phys. Lett. 119, 259 (1985).

7. David P. Shoemaker, Carl W. Garland, Jeffrey I. Steinfeld, and Joseph $w$. Nibler, Experiments in Physical chemistry, 4th ed. (MCGraw-Hill, New York, 1981) pp. 708-737.

8. William H. Press, Brian P. Flannery, Saul A. Teukolsky, and William T. Vetterling, Numerical Recipes in $C$ : The Art of scientific computing, (Cambridge University press, Cambridge, 1988), pp. 523-525.

9. P.M. Rentzepis, Chem. Phys. Lett. 3, 717 (1969).

10. Dieter Klemp and Bernhard Nickel, Chem. Phys. Lett. 130, $493(1986)$.

11. R.J. Sension, S.T. Repinac, and R.M. Hochstrasser, J. Chem. Phys. 93, 9185 (1990).

12. A.L. Harris, M. Berg, and C.B. Harris, J. Chem. Phys. 84, 788 (1986).

13. Aviv Amirav and Joshua Jortner, J. Chem. Phys. 81, $4200(1984)$.

14. David Chandler, Introduction to Modern Statistical Mechanics, (Oxford Univ. Fress, New York, 1987) and accompanying lecture notes.

15. M.E. Paige and C.B. Harris, J. Chem. Phys. 93, 3712 (1990); M.E. Paige and C.B. Harris, Chem. Phys. 149, 37 $(1990)$. 
16. William G. Dauben, University of California - Berkeley, Berkeley, CA, personal communication (1991).

17. L.C. Gruen and F.A. Long, J. Amer. Chem. Soc. 89, 1287 (1967).

18. Takehiro Shimanouchi and Isao Suzuki, J. Molec. spectroscopy, 6, 277 (1961).

19. J.H. Schachtschneider and R.G. Snyder, spectrochim. Acta 19, 117 (1963).

20. U. Sukowski, A. Seilmeier, T. Elsaesser, and S.F. Fischer, J. Chem. Phys. 93, 4094 (1990). 
Chapter 4: ANALYSIS

4.1 Vibrational Relaxation studies of $I_{2}$ in compressed Gases

Previous studies ${ }^{1,2,3}$ of the relaxation of geminately recombined, vibrationally hot $I_{2}$ in compressed noble gases provide a system for comparison with this work on azulene cooling dynamics. The experimental conditions are identical to the conditions in this current work. The same pressures and temperatures were used in the iodine and azulene experiments. The only difference is the solute and the amount of internal energy to be lost to the solvent. Newly recombined iodine atoms must dissipate approximately 14,000 $\mathrm{cm}^{-1}$ of excess energy to reach equilibxium, slightly less than the $17,000 \mathrm{~cm}^{-1}$ of energy in a hot ground state azulene molecule.

The results of the iodine study were strikingly different from the results obtained for azulene. Iodine requires $3-12$ nanoseconds to cool in liquid xenon, yet azulene relaxes almost ten times faster. Both solutes exr mience an increase in the vibrational cooling rate, by a factor of 10 for azulene and a factor of 20 for $I_{2,}$ when in chloromethanes and hydrocarbons instead of compressed noble gases. ${ }^{2}$ This was attributed to the lack of internal modes in xenon to accept energy from the hot solute as well as the lack of coupling between the low frequency solvent modes with the vibrational frequency of $I_{2} \cdot{ }^{1}$ similarly, the 48 vibrational modes of 
azulene provide more opportunity to lose energy, although only the very lowest fxequency modes would couple well to the low frequency modes of xenon and facilitate energy transfer to the solvent. This could explain why the relaxation speeds up slightly in krypton, because it has higher frequency acceptor modes that overlap better with vibrational modes in azulene.

The vibrational celaxation of iodine demonstrates a dramatic density dependence whereas azulene does not. Between the highest xenon density, $3.0 \mathrm{~g} / \mathrm{ml}$, and the lowest, $1.8 \mathrm{~g} / \mathrm{ml}$, the relaxation rate of geminate iodine molecules decreases by a factor of three. ${ }^{3}$ This change is very close to the results calculated theoretically using Isolated Binary Collision theories and molecular dynamics simulations. Isolated Binary collision theory predicts a three fold rate change which should be entirely due to and linearly correlated with the change in collision frequency with density. Results from molecular dynamics simulations showed that the collision frequency at a physically reasonable I-Xe contact distance was three times larger in the high density xenon solution than in the low density xenon solution. These theories seem to reasonably predict the vibrational cooling behavior of geminately recombined iodine atoms. ${ }^{2}$

Iodine shows an approximately twofold increase in the cooling rate with a change in temperature froin $253 \mathrm{~K}$ to $323 \mathrm{~K}$. In some ways this result is surprising since the experimental temperature change would be only a minor perturbation to a 
molecule with $12,500 \mathrm{~cm}^{-1}$ of internal energy. The collision contact distance between $I_{2}$ and $X e$ may shrink somewhat at higher temperatures, which will change the collision frequency and thus the relaxation rate. However, it appears that the hotter solvent atoms must collisionally deactivate the iodine molecule more efficiently since molecular dynamics simulations indicate that the change in collision frequency over this temperature range is insignificant. This temperature effect on the cooling rate of $I_{2}$ may be specifically related to the iodine molecule itself. In fact, the minimal effect of temperature on vibrational relaxation seen in azulene is perhaps what is to be expected unless the temperature change is much larger. Further controlled temperature studies are needed to illuminate the effect of temperature on vibrational relaxation.

The results of the iodine experiments can be converted into a plot of average energy lost to the solvent as a function of time. The resulting plot shows a bottleneck in the vibrational relaxation process, presumably due to the increasing mismatch between the $I_{2}$ vibrational frequency (150 - $220 \mathrm{~cm}^{-9}$ ) and the very low frequency solvent translational. modes. In the upper half of the ground state iodine potential, vibrational relaxation occurs quickly, in $<150$ psec. In the lower half of the well, relaxation is slower, $>3000$ psec. $^{2}$ This demonstrates the sensitivity of energy transfer between a vibrational mode and the surrounding 
solvent bath modes. There is the possibility of a bottleneck in the energy flow from azulene to the solvent. Since visible transient absorption data only probes some of the vibrational levels in the molecule, it can not determine whether the energy is flowing directly into the solvent or into some other modes in the molecule, which are not detected by transient absorption. It is assumed that intramolecular vibrational enexgy redistribution (IVR) is rapid ${ }^{21}$ and that all modes are coupled well enough to make a bottleneck of energy in a single mode unlikely. 4

\subsection{Isolated Binary Collision Theories}

Isolated Binary collision (IBC) theories are conceptually simple theories which provide a physical picture to describe collisional energy transfer in the condensed phase. Having derived from gas phase theories, IBC theories take advantage of information that has been previously obtained from gas phase experiments. They have also been used successfully to describe vibrational relaxation in many different systems. This work tests the application of IBC theory to describe the relaxation of vibrationally excited polyatomic molecules.

Theoretical predictions of the effect of solvent on vibrational cooling lifetimes can be made using Isolated Binary collision theory. As the name implies, this theory assumes that isolated two body collisions between solute and solvent are most important in deactivating a molecule. 
Multibody effects are neglected. It assumes that the cross section of collisions in a gas and a liquid are the same and that the difference in relaxation rates in the two media is due to the difference in collision frequencies, collisions occurring more often in the condensed media. IBC theor $\mathrm{V}$ takes advantage of energy transfer information from gas phase relaxation studies to describe the amount of energy transferred in a collision.

The basis of IBC theory is that the rate of relaxation (K) can be separated into the product of two components, the collision frequency $\left(v_{c}\right)$, which is dependent on solvent density $(\rho)$ and temperature (T), and the probability of energy transfer between two levels per collision (P), which is density independent (Eqn. 4.1).5

$$
K(\rho, T)=v_{c}(\rho, T) \cdot P(T)
$$

Thus any change in the relaxation rate with density must be due to changes in collision frequency.

In order to use IBC equations, the collision frequency must be determined. However, it is difficult to define what a collision is in a condensed media where molecules are tightly packed and often strongly interacting with one another. This makes it hard to calculate the frequency of collisions occurring in the medium. There have been a number of attempts to calculate collision frequencies using model descriptions of a liquid environment. one of the most 
successful models that has been used to calculate liquid phase collision frequencies is that pro posed by Davis and oppenheim, which provides a simple physical picture of collisions in liquid and incorporates structural information about the liquid.

Davis and oppenheim modeled the collision frequency in a liquid as a flux of colliding solvent molecules through a spherical surface, of area $4 \pi \mathrm{R}^{2}$, surrounding the solute. The flux is proportional to the velocity of solvent molecules ( $v$ - $\left.\left(k_{B} T / \pi \mu\right)^{1 / 2}\right)$ multiplied by the local density of solvent molecules, $p g(R)$ (the radial distribution function), evaluated at $R^{*}$, the most effective collision radius between solute and solvent molecules. This results in a collision frequency: ${ }^{6}$

$$
v_{C}=4 \pi R^{* 2} \rho g\left(R^{*}\right) \quad\left(k_{B} T / \pi \mu\right)^{1 / 2}
$$

The implicit assumption in Eqn. (4.2) is the spherical eymmetry associated with $R^{2}$ and $g(R)$. It is not at all clear that such an assumption is valid for anisotropic molecules, such as azulene.

The value of $P$, the probability of energy transfer per collision, is solvent dependent and has been determined by gas phase relaxation studies of azulene in krypton and xenon buffer gases, using IR emission and hot UV absorption spectroscopy. ${ }^{7,8}$ These results are listed in Table 4.1 (identical to Table 1.2) and can be applied to the various experimental conditions that have been studied. 
Table 4.1 Azulene-buffer gas energy transfer values ( $\Delta E$ in $\left.\mathrm{cm}^{-1}\right)$ at three different internal energies $\left(\langle E\rangle-\left\langle E_{T}\right\rangle\right) \cdot \Delta E$ is proportional to P. Tabular data from Reference 7.

\begin{tabular}{|c|c|c|c|c|}
\hline & & $\Delta \mathrm{E}$ & $\langle E\rangle-\left\langle E_{T}\right\rangle$ & $\left(\mathrm{cm}^{-1}\right)$ \\
\hline Solvent & Temp. (K) & 5000 & 10000 & 15000 \\
\hline $\mathrm{Ne}$ & 298 & 60 & 100 & 130 \\
\hline Ar & 298 & 70 & 120 & 170 \\
\hline $\mathrm{Kr}$ & 298 & 60 & 120 & 160 \\
\hline $\mathrm{Xe}$ & 298 & 50 & 120 & 170 \\
\hline $\mathrm{Ne}$ & 410 & 35 & 65 & 95 \\
\hline Ar & 600 & 50 & 80 & 85 \\
\hline $\mathrm{Kr}$ & 670 & 40 & 70 & 100 \\
\hline $\mathrm{Xe}$ & 830 & 40 & 80 & 110 \\
\hline
\end{tabular}


The gas phase studies indicate that the difference in the average amount of energy transferred per collision in xenon and krypton is very small and that the ratio of $P$ in krypton to that in xenon is approximately unity (i.e. $\mathrm{P}_{\mathrm{kr}} / \mathrm{P}_{\mathrm{xe}} \approx 1$ ). ${ }^{7}$ Therefore according to Eqn. (4.1), the difference in dynamics must be due to differences in the collision frequency. By using Eqn. (4.2) and ratioing the collision frequency in krypton to that in xenon, various terms cancel out and simplify the result. The difference in $R^{*}$ in xenon and krypton can be approximated by the difference in the LennardJones interaction distance, $\sigma$, given in Table 4.2. The variation of $R^{*}$ in the solvents is small and the ratio $\left(R_{k r}{ }^{*}\right)^{2} /\left(R_{k e}{ }^{*}\right)^{2}$ is 0.93 . There should also be little difference in $\rho g\left(R^{*}\right)$ for $\mathrm{Xe}$ and $\mathrm{Kr}$ at the same reduced density. The term $\rho g\left(R^{*}\right)$ describes the number density of solvent atoms surrounding the azulene at a distance $R^{*}$ from the azulene. Because of the similarity of krypton and xenon, this packing should be similar and the ratio $\left[\rho g\left(R^{*}\right)\right]_{k r} /\left[\rho g\left(R^{*}\right)\right]_{x e}$ should be approximately unity or somewhat greater. The differences in the relaxation rate in the two solvents should be primarily aue to the velocity term $\left(k_{B} T / \pi \mu\right)^{1 / 2}$. Therefore, the ratio of $\mathrm{K}_{\mathrm{kr}} / \mathrm{K}_{\mathrm{ke}}$ should be proportional to $\left(\mu_{\mathrm{xe}} / \mu_{\mathrm{kr}}\right)^{1 / 2}$, approximately 1.1. Experimentally, the values of $\mathrm{K}_{\mathrm{kr}} / \mathrm{K}_{\mathrm{ke}}$ are $1.3 \pm 0.3$ at the low density and $1.5 \pm 0.2$ at the higher density. The predicted ratio of the collision frequencies and the experimental ratios are not in close agreement but do show that IBC theories are 
generally applicable to this system.

The temperature dependence of vibrational relaxation is more difficult to determine using IBC theories because both the collision frequency and the energy transfer per collision are dependent upon temperature (see Eqn. (4.1)). However, the value of $P$ for azulene in noble gases at different temperatures can again be obtained from gas phase studies (see Table 4.1), which show that the temperature dependence of $P$ over the experimental range of temperatures is minimal (i.e. $\left.\mathrm{P}_{253 \mathrm{~K}} / \mathrm{P}_{323 \mathrm{~K}} \approx 1\right) \cdot{ }^{7}$ Therefore the temperature dependence of the cooling of azulene predicted by IBC theory would be solely due to the ratio of the collision frequencies defined in Eqn. (4.2). Neglecting any minor temperature dependence in $R^{\star}$ and $g\left(R^{*}\right), \quad K_{253 K} / K_{323 K}$ should equal $(253 \mathrm{~K} / 323 \mathrm{~K})^{1 / 2}$ which is approximately 0.88 . The cooling times for the four different temperatures fall in a range of values within two standard deviation units of the average, the two most extreme values having a ratio of 0.89 , very near the value predicted above. IBC theory predicts the minimal temperature dependence seen experimentally.

Isolated Binary Collision theory is quite useful in describing the temperature effect on the relaxation of azulene. The assumptions made above to apply IBC theories are quite reasonable. There may be some decrease in $\mathrm{R}^{*}$ with temperature because the molecules are moving faster and thus collisions are harder and more efficient, however the 
temperature dependence in $R^{*}$ should be minor over this small temperature range. In general, the radial distribution function, $g(R)$, of simple liquids shows sharper peaks and more structure with increasing temperature. However, the slope of $g(R)$ at the two extreme temperatures in the region of $R^{*}$ remains relatively parallel.9 If $g(R)$ at two temperatures are ratioed, they would cancel each other out, thus justifying the assumption made above. IBC theories quite accurately model the effect of solvent temperature on the cooling dynamics of azulene in noble gas liquids.

As noted previously, the relaxation rate in xenon only changes by $20 \%$ when the reduced density changes from 0.57 to 0.95. According to IBC theories (Eqn. (4.1)), the density effect on the relaxation should be entirely due to the change in collision frequency at the two different densities, since the probability terms are density independent. Although the value of $R^{*}$ may be expected to decrease slightly as the solvent density increases, this effect is probably minor and can be neglected. It is the explicit density dependence of the collision frequency, shown in Eqn. (4.2), in the term describing the local density of solvent around the solute, $\rho g\left(R^{*}\right)$ which determines the solvent density effect on collisions. The value of $\rho$, the number density of the bulk solvent, is determined empirically from experimental conditions. The value of $g(x)$ can be obtained from molecular dynamics simulations. If one assumes a Lennard-Jones 
interaction between the azulene and xenon, the resulting radial distribution function is almost identical to that obtained in the iodine/xenon studies, ${ }^{10}$ carried out under the same experimental conditions, the same solvent densities and temperatures.

If the value of the bulk density is identical in the azulene and the lodine experiments, and the value of the $g\left(R^{*}\right)$ is the same for the azllene and iodine studies, Eqn. (4.2) would predict that the collision frequencies and thus the trend of relaxation rates as a function of density should be identical. However, empirically, the effect of density on the relaxation rates is strikingly different in the two studies, solvent density having a minor effect on azulene relaxation and having a major impact on iodine relaxation (see section 4.1 for comparison).

This apparent density independence in the azulene cooling time is much more difficult to explain than the temperature or solvent mass dependence. One possibility is the effect of the three dimensional anisotropy of the solute leading to breakdown of the spherical symmetry approximation, cited earlier. The terms $g(R)$, the radial distribution function, and $R^{*}$, the deactivation distance are calculated for spherical molecules. IBC models worked very well in describing the dynamics of iodine, a molecule which might fit the IBC assumptions better than a poiyatomic, planar molecule like azulene. 
If the rare gas atoms packed above and below the azulene ring systems, then a complex three dimensional radial distribution function would be required in order to use IBC theories. In fact, experimental evidence from gas phase cluster studies indicate that this is exactly the binding geometry between aromatics like azulene and noble gases." Although the exact placement of solvent relative to the azulene ring system is not yet known, Figure 4.1 indicates the relative size of azulene ${ }^{7}$ and $x_{e n o n}{ }^{14}$ (to scale) and shows some possible packing configurations of azulene and xenon. It is also possible that two xenon atoms may sit above and below the two carbon rings in azulene.

Assuming that the solvent asymmetrically packs around the azulene, the value of $R^{*}$ would necessarily be a complex three dimensional function, dependent upon the geometry and local density of the solvent. Although difficult to show in the absence of a complete calculation, one might imagine that the terms $R^{*}$ and $g\left(R^{*}\right)$ could compensate each other for changes in the value of $\rho$. This would result in an apparent density independence in the dynamics. Alternatively, collisions along one direction of the molecule may be important in deactivating it and yet show no density effect. Currentiy Isolated Binary collision Theory can not fully incorporate these three dimensional anisotropic terms into calculations, which leads to inexact descriptions of real molecular systems.

Three dimensional molecular dynamics simulations of 
A)

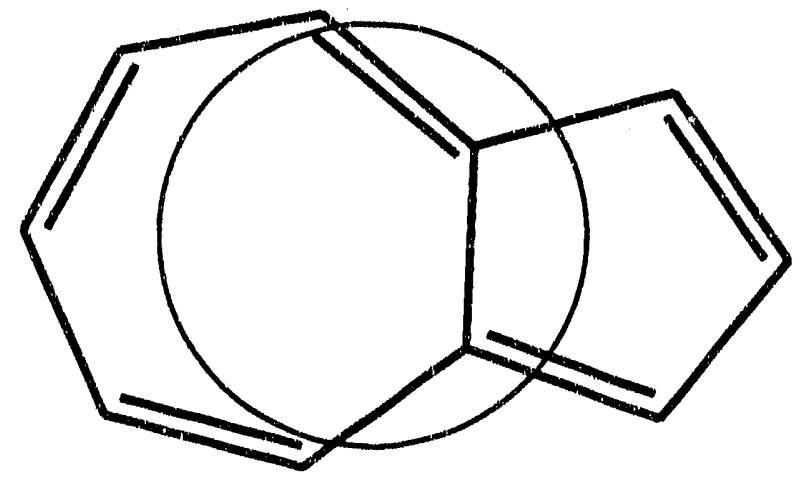

\section{drawings to scale}

B)

Pigure 4.I Relative size of azulene and xenon 
azulene in liquid xenon are currently underway in an attempt to illustrate the geometric effect described above and apply it to IBC theories. 12 These simulations are static calculations of the packing geometry, that is, the azulene is not considered to be vibrationally excited. Preliminary evidence indicates that the density of solvent atoms above and below the azulene rings does not change as a function of density although the density in the plane of the rings does. Low frequency out-of-plane bending modes are assumed to drain energy from the azulene to the solvent since these low frequencies overlap with the low frequency solvent modes. since these out-of-plane modes essentially see little solvent density change over the experimental range, it is understandable that the overall relaxation of the azulene would be independent of solvent density.

Further experimental studies and three dimensional molecular dynamics simulations of complex molecules in solution would further the current understanding of the density effect on energy transfer. As well, simulations which can provide structural information $(g(R))$ for IBC equations would make it easier to apply these models to more common chemical systems.

\subsection{Molecular Dynamics Simulations of Solvent Effects}

It is experimentally difficult to cleanly examine the effect of solvent mass on reaction dynamics. Isotope studies 
are the best way to do this since the mass, and not the potential, is the only difference between two isotopes. Unfortunately, the mass change between most isotopes is too small to make a difference. The closest equivalent to isotopes are isovalent molecules within one group of the periodic table, such as the noble gases. Withill a group, the chemistry is similar though the masses differ greatly between elements. Comparing the dynamics of azulene in xenon and krypton is dependent upon the assumption that the largest effect on the dynamics in $\mathrm{Xe}$ and $\mathrm{Kr}$ should be due to the difference in the solvent mass.

The cleanest way to study the effect of solvent mass on dynamics is by way of molecular dynamics simulations. Using molecular dynamics simulations, one can accurately examine the effect of solvent mass on the dynamics in question by simply changing the value for the solvent mass in the simulation coce and leaving all other parameters constant. Results from simulations of this sort provide information about the effect. of solvent on vibrational relaxation.

Simulations of the $I_{2} /$ Xe solutions mentioned earlier were conducted using Lennard-Jones potentials to describe the I-Xe interaction. The details of these simulations have been described elsewhere ${ }^{13}$ and the results are summarized here for comparison to the azulene results. Simulations tested the effect of solvent mass by using a fictitious solvent with half the mass of a xenon atom. simulations also tested the 
sensitivity of the results to the interaction potential between iodine and xenon atoms by using a solvent with a well depth of $50 \%$ the standard Lennard-Jones I-Xe interaction. This lattier change also has the effect of substantially softening the repulsive wall.

The result of using a half mass solvent was to increase the rate of the relaxation process by a factor of two. This was experimentally borne out by the results from iodine relaxation in chloromethanes ( $I_{2}$ does not dissolve in liquid krypton), where the ratio of the masses of $\mathrm{CH}_{2} \mathrm{Cl}_{2}$ and $\mathrm{CCl}_{4}$ is equivalent to the ratio of the vibrational relaxation times. similarly in the azulene work, the ratio of the masses of Xe and $\mathrm{Kr}$ is approximately $3: 2$. The ratio of the vibrational cooling lifetimes is $175: 118$ or 1.5 at high density and 1.3 at low density. These results follow the trend seen in the simulation results for $I_{2}$ in liquid xenon. Tnis lends confidence to the statement made above that the noble gases, krypton and xenon, affect the relaxation dynamics most notably due to their mass and to a lesser extent, their potentials.

However, the difference in the interaction potential of the two noble gas solvents may be interfering with the comparison of the dynamics in these solvents. Simulation results comparing two xenon solvent:; one with half the Lennard-Jones well depth of the other, indicate that the vibrational relaxation results for $I_{2}$ are sensitive to solvent interaction potential. The relaxation process is 
significantly slower in the reduced well depth system due to the softer repulsive wall. The Lennard-Jones potential parameters for the $I_{2} /$ Xe simulations are tabulated along with the azulene/Xe and azulene/Kr Lennard-Jones parameters in Table 4.2 .14

A comparison of the curvature of the azulene potentials and the iodine potentials shows the dramatic effect of reducing the well depth by $50 \%$. One can see that the repulsive wall is significantly less steep in the altered iodine potential, as shown on the left in Figure 4.2 which shows the two iodine potentials overlapped at their minima. This can be compared to the azulene-noble gas potentials, shown on the right in Figure 4.2 overlapped at their minima. This figure makes it very obvious that the noble gas potentials are similar in terms of the steepness of the repulsive wall. It is the steepness of the wall which influences dynamics in liquids with reduced densities of 0.6 or greater, ${ }^{15}$ as in this study. Because the noble gas potentials are so similar, the difference in the vibrationaj. cooling behavior in the two noble gas solvents is largely due to changes in the mass and not in the potential. The behavior that is seen empirically for azulene in noble gas solvents is entirely consistent with the simulation results for iodine in xenon.

\subsection{Bulk Thermal Diffusion Theories}


Table 4.2 Lennard-Jones potential parameters for molecular interactions cited in text.

\begin{tabular}{|c|c|c|}
\hline \multirow{2}{*}{$\begin{array}{l}\text { Molecular } \\
\text { Interaction }\end{array}$} & \multicolumn{2}{|c|}{ Lennard-Jones Parameters } \\
\cline { 2 - 3 } & E $(\mathrm{K})$ & $\sigma(\mathrm{A})$ \\
\hline $\mathrm{I}-\mathrm{Xe} \mathrm{(standard)}$ & 324 & 3.94 \\
\hline $\mathrm{I}-\mathrm{Xe} \mathrm{(altered)}$ & 162 & 3.94 \\
\hline $\mathrm{Az}-\mathrm{Xe}$ & 346 & 5.33 \\
\hline $\mathrm{Az}-\mathrm{Kr}$ & 305 & 5.13 \\
\hline
\end{tabular}

lodine parameters from Reference 13.

Azulene parameters calculated from noble gas self potential parameters in Reference 14 and azulene self potential parameter in Reference 7 using equations from Reference 8 . 


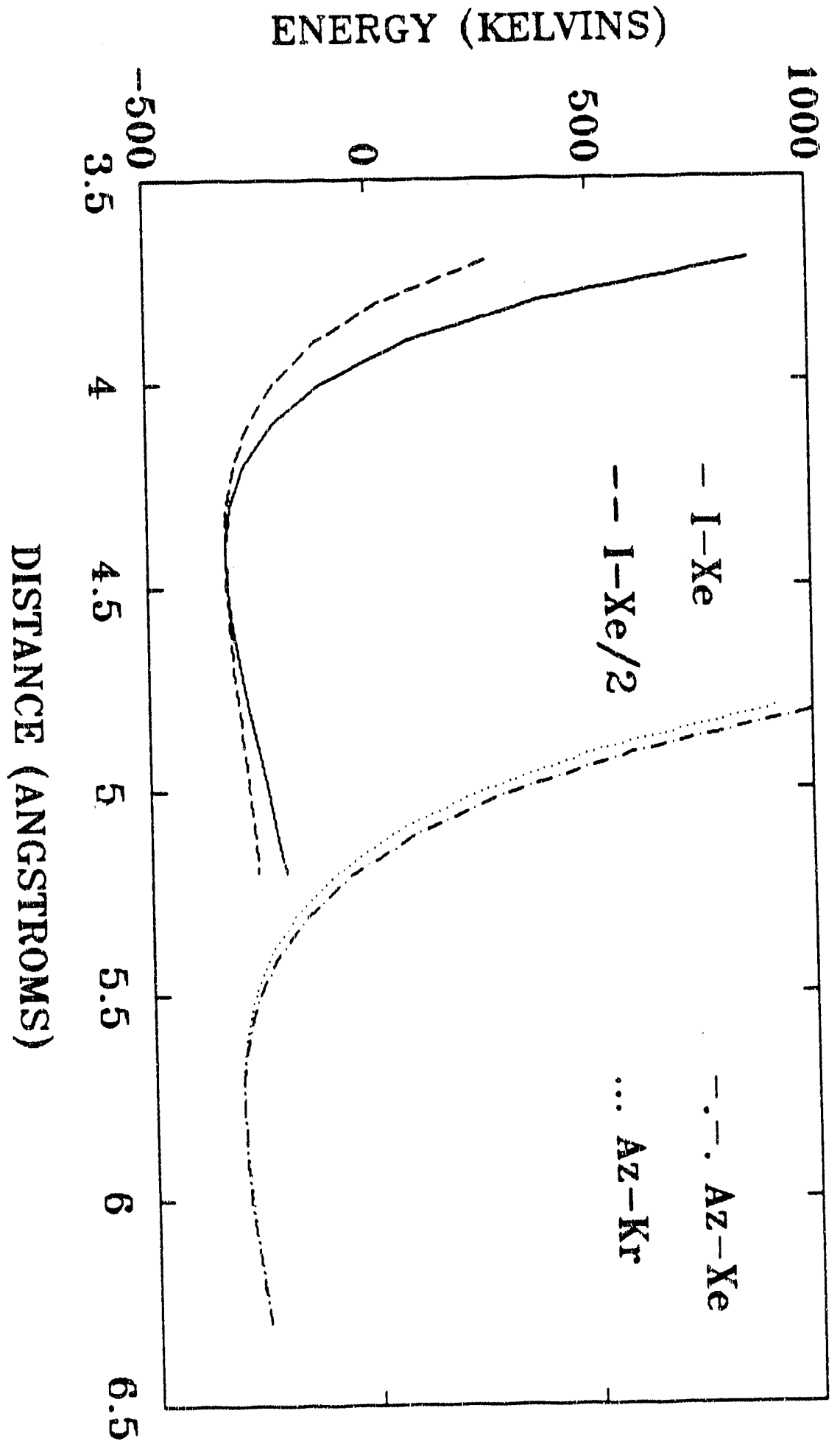

Figure 4.2 Lennard-Jones interaction potentials overlapped at minima. (right) Iodine-xenon potentials used in simulations (see text). Azulene-noble gas potentials. (left) 
The most simplistic way to think of the vibrational cooling of an azulene molecule in a liquid is like the cooling of a hot stone dropped in a pool of water. The hot stone loses its heat to the quenching bath around it until it reaches equilibrium with the surrounding bath. This scenario is an example of bulk thermal cooling models. The time dependence of the internal temperature is exactly the vibrational cooling rate that we seek. others have successfully used this model to describe the loss of energy from a large aromatic solute molecule into the solvent. $16,17,18,19$ Therefore, it seemed that this simple approximation may apply to this system also.

First, the internal temperature of the molecule must somehow be related to the absorption spectrum or some other experimental parameter that can be quantified and measured as a function of time. This relationship can be determined in a direct manner with a few assumptions. The molecular system is described as a dense equidistant distribution of vibrational states with equal Franck-Condon factors; ${ }^{17}$ that is, the m-lecule can be approximated as a series of relatively uncoupled harmonic oscillators. This is a very simple approximation for a real molecule, however it is not entirely unreasonable. The extinction coefficient, $\epsilon$, of the transition between thermally populated ground state vibrations to the first excited state is proportional to the product of $N_{t}$, the number of thermally excited molecules, and $\sigma_{\text {tran }}$ the 
cross section for the given transition. $N_{t}$ can be written as the product of $\mathrm{N}_{0}$, the number of molecules, and $\exp (\mathrm{h}(\mathrm{v}-$ $\left.v_{0}\right) / k_{B} T$ ), the Boltzmann factor where $T$ is internal molecular temperature and $v-v_{0}$ is the difference between the $0-0$ frequency and probe frequency i.e. it is the hot band frequency. This results in Eqn. 1 of reference 17:

$$
\boldsymbol{\epsilon}=\log _{10}(e) \boldsymbol{\sigma}_{\text {tran }} N_{0} \exp \left(h\left(v-v_{0}\right) / k_{B} T\right)(4.3)
$$

A spectral shape factor was added to this equation in reference 16 to account for variations in the Franck-Condon factors in the transitions, eliminating part of the earlier assumption. Without this shape factor, the Franck-Condon factors are assumed to be equal for all hot band vibrational transitions.

Differentiation of Eqn. (4.4) predicts a linear relationship between the relative change in the extinction coefficient with respect to temperature and the difference in frequency, as in Eqn. (2) of reference 17:

$$
(d \boldsymbol{\varepsilon} / \varepsilon) / d T=C\left(v-v_{0}\right) \text { where } C=h / k_{B} T^{2(4.4)}
$$

Kaiser experimentally determined that this relation was true for vibrationally hot azulene. ${ }^{18}$ Tigure 2 of reference 18 shows an increase in the ground state absorption spectrum after excitation. When fit with Eqn. (4.4) above, a temperature of $1200 \mathrm{~K}$ was obtained which is reasonable considering the amount of energy present in the molecule. Our 
calculation of the internal energy of azulene upon photoexcitation at $590 \mathrm{~nm}$ (as in this work) using the density of states (see Appendix for program used) yielded a temperature of $1100 \mathrm{~K}$. This substantiates Kaiser's experimental result.

From Eqn. (4.4) at two different temperatures, $T_{0}$ and $T_{1}$, the relative change in the extinction coefficient $(\epsilon)$ can be calculated. This is proportional to the relative change in the absorption coefficient $(\alpha)$ which is measured experimentally. The result is Eqn. (4) from reference 16 (Eqn. $(4.5))$ :

$$
\begin{aligned}
& {\left[\alpha\left(v, T_{1}-\alpha\left(v, T_{0}\right)\right] / \alpha\left(v, T_{0}\right)=\right.} \\
& K\left[\exp \left[h\left(v-v_{0}\right)\left(\left(k_{B} T_{1}\right)\right)^{-1}-\left(k_{B} T_{0}^{-1}\right)\right]-1\right]
\end{aligned}
$$

A simpler version of this is Eqn. (4.6), from reference 19, where the term $E_{v}$ is the energy difference between the $0-0$ transition and the probe transition i.e. $E_{v}$ is the frequency of the hot band being probed.

$$
\begin{gathered}
\Delta \alpha / \alpha=\exp \left[E_{v}\left(1 / k_{B} T_{1}-1 / k_{B} T_{0}\right)\right]-1 \\
\text { where } E_{v}=h\left(v-v_{0}\right)
\end{gathered}
$$

Notice that Eqn. (4.6) above does not contain any time dependence. The time dependence comes into the temperature term. It is the temperature of the molecule that decays in time due to collisional energy loss to the solvent. The time dependent behavior of the temperature is calculated by means 
of bulk thermal cooling equations. This process of non-steady state heat flow has long been known to engineers. As a result, the equation describing thir scenario is well defined when simple assumptions are made. 20

The assumptions implicit in this model are that flow of heat out of the hot solid and into the liquid is the dominant process and that heat flow through ths solid itself is quite rapid. These assumptions are reasonable for the azulene molecule since it is extremely hot at excitation, about $1100 \mathrm{~K}$, causing heat loss to be the dominant mechanism, which satisfies the first assumption. Secondly, IVR occurs repidly in aculene," presumably because of the high molecular symmetry which provides efficient mode coupling, thus satisfying the second assumption. Bulk thermal cooling equations to describe the loss of energy from a large aromatic solute molecule into the solvent have been used in the past by other researchers. ${ }^{19}$ However, these researchers never sought to relate the cooling time to parameters of the solvent such as thermal conductivity. This neglects the role that the solvent plays in dispersing energy from a hot solute molecule. Incorporating this idea into the current work is not difficult.

The differential equation that links the internal temperature of the molecule with time is Eqn. (4.7), which incorporates azulene parameters and solvent parameters: $C_{p}$ is the specific heat of the solid, $p$ is the density of the 


$$
\frac{d T}{T-T_{\infty}}=-\frac{h A_{s}}{C_{p} \rho V} d t
$$

solid, $\mathrm{V}$ is the volume of the solid, $\mathrm{A}_{\mathrm{s}}$ is the surface area of the solid, $T$ is the average temperature of the solid, $T_{0}$ is the initial temperature of the liquid, $h$ is the average heat transfer coefficient of the liquid and $t$ is time. The solution to this aifferential equation is shown in Eqn. (4.8)

$$
\frac{T-T_{\infty}^{\prime}}{T_{0}-T_{\infty}}=e^{\frac{-h_{A} A_{s}}{C_{p} \rho V}}
$$

where the $T_{0}$ is the initial temperature of the soidd and all other parameters are as described above. ${ }^{20}$

To simplify the equation further, assume a small spherical shape for the solid being cooled, i.e. the azulene molecule. This is a poor assumption for a planar aromatic such as azulene; however it is a first order approximation. Then $A_{b} / V=3 / r$, where $r$ is the radius of the sphere and $h=$ $k / r$ where $k$ is the thermal conductivity of the liquid, averaged between the temperature extremes $T_{0}$ and $T_{\infty}$. There is also the implicit assumption that the thermal conductivity of the liquid is averaged over the two extreme temperatures, $T_{0}$ and $T_{w}$, or in other words, that the thermal conductivity of the liquid is linearly dependent upon temperature. It is likely that the surrounding solvent does not reach the high temperature $\left(T_{0}\right)$ that the azulene does, ${ }^{25}$ however in the absence of the exact solvent temperature, $T_{0}$ is used as a first approximation. This greatly simplifies the 
calculations. 20

The solution to the differential equation simplifies to Eqn. $(4.9):$

$$
T^{\prime}=\left(T_{0}-T_{\infty}\right) \exp \left(-3 k / C_{p} \rho I^{2}\right) t+T_{\infty}
$$

This equation predicts the time dependence of the temperature of the azulene. The term $c_{p} p r^{2} / 3 k$ has units of time and is the time for the temperature of the azulene to drop $1 / e$. The exponential relaxation is seen in the vibrational cooling data and predicted by theory. 22

This equation is very similar to Eqn. (2) in reference 1y, except what the authors label the term $c_{p} \mu r^{2} / 3 k$ as $\tau$ and do not use solvent parameters in this term. Instead, they use it as an adjustable parameter. However, it is this term which contains parameters involving the solvent and it is this term which partially dictates the decay time. This is not the only term which influences the decay of the molecular internal temperature; probe frequency, contained in $\mathrm{E}_{v}$, and the initial and final temperature also will affect the decay time.

Substituting azulene values for the physical constants in Eqn. (4.9) gives a theoretical lifetime for the process of vibrational cooling. The initial temperature of the liquid $\left(T_{\infty}\right)$ is assumed to be $280 \mathrm{~K}$ for the compressed gas solvents, room temperature for all other solvents. The initial temperature of the azulene is assumed to be $1100 \mathrm{~K}$. The value for the radius of the azulene sphere is the half the Lennard- 
Jones diameter or $3.3 \AA .^{7}$ The heat capacity of azulene is a function of temperature but is assumed to be constant in this calculation. This will have some effect on the trend in the results, particularly the temperature studies, however its largest effect will be on the absolute magnitude of the fit. The value of $c_{p}$ at $280 \mathrm{~K}$ is $928 \mathrm{Jkg}^{-1} \mathrm{~K}^{-1} \cdot 23$ Because of the approximations made for this model, the absolute value of the lifetime from this calculation will not be exact. In fact, the absolute value of the calculated lifetime must be altered by nany orders of magnitude in order to use with the actual data. These approximations will affect the accuracy of the final results. However, the trend of lifetimes in a series of experimental conditions should be accurate.

There are several parameters which will affect the fit of the data to the theoretical model. There is the value of the energy term $h\left(v-v_{0}\right)$ which is in the exponential. As one changes the probe frequency (v) the value of the energy term changes, becoming larger as $v$ moves farther from the origin $\left(v_{0}\right)$ and probes higher frequency hot bands. In fact, this model supports the wavelength dependence seen in the azulene vibrational cooling times very well. The lower frequency probe wavelengths presumably probe highex energy populations and higher frequency modes. These populations lose energy more quickly to lower energy modes, probed by wavelengths closer to $v_{0}$ and thus these low frequency probes decay faster. Higher frequency hot bands increase the term $v-v_{0}$ in Eqn. 
(4.6) and therefore the calculated lifetime will be shorter when probing these hot bands. This trend is predicted by the model, with the experimental results and predicted fits shown in Figure 4.3 .

In most of these experiments in compressed gases, the probe wavelength is fixed at $740 \mathrm{~nm}$. This means thit the energy term $h\left(v-v_{0}\right)$ is not really a variable parameter for the fit. The thermal conductivity values axe obtained from thermodynamic data for the specific experimental conditions. ${ }^{24}$ These are listed for various solvents in Table 4.3. In this sense, they are not variable parameters either. The only variable parameter in the system is the amplitude of the fit relative to the data, caused by errors in the approximations of the model, stated earlier.

When the thermodynamic values for the thermal conductivity are put in the model, the amplitude of the fit is varied until the best $f i t$ is obtained. This is done for each experimental condition. The two to four scans that make up an experimental test condition (temperature, density, wavelength, etc...) are shown together normalized at the peak with the best fits in Figure 4.3 - Figure 4.7. The theoretically predicted lifetimes for the internal temperature of the azulene $\left(c_{p} \rho r^{2} / 3 k\right)$ are listed in Table 4.4 along with the experimental cooling lifetimes. The theoretical lifetimes are used in Eqn. (4.9) which calculates the temperature change for use in Eqn. $(4.6)$ to calculate the absorbance change. The 
$\%$ CHANGE IN ABSORBANCE

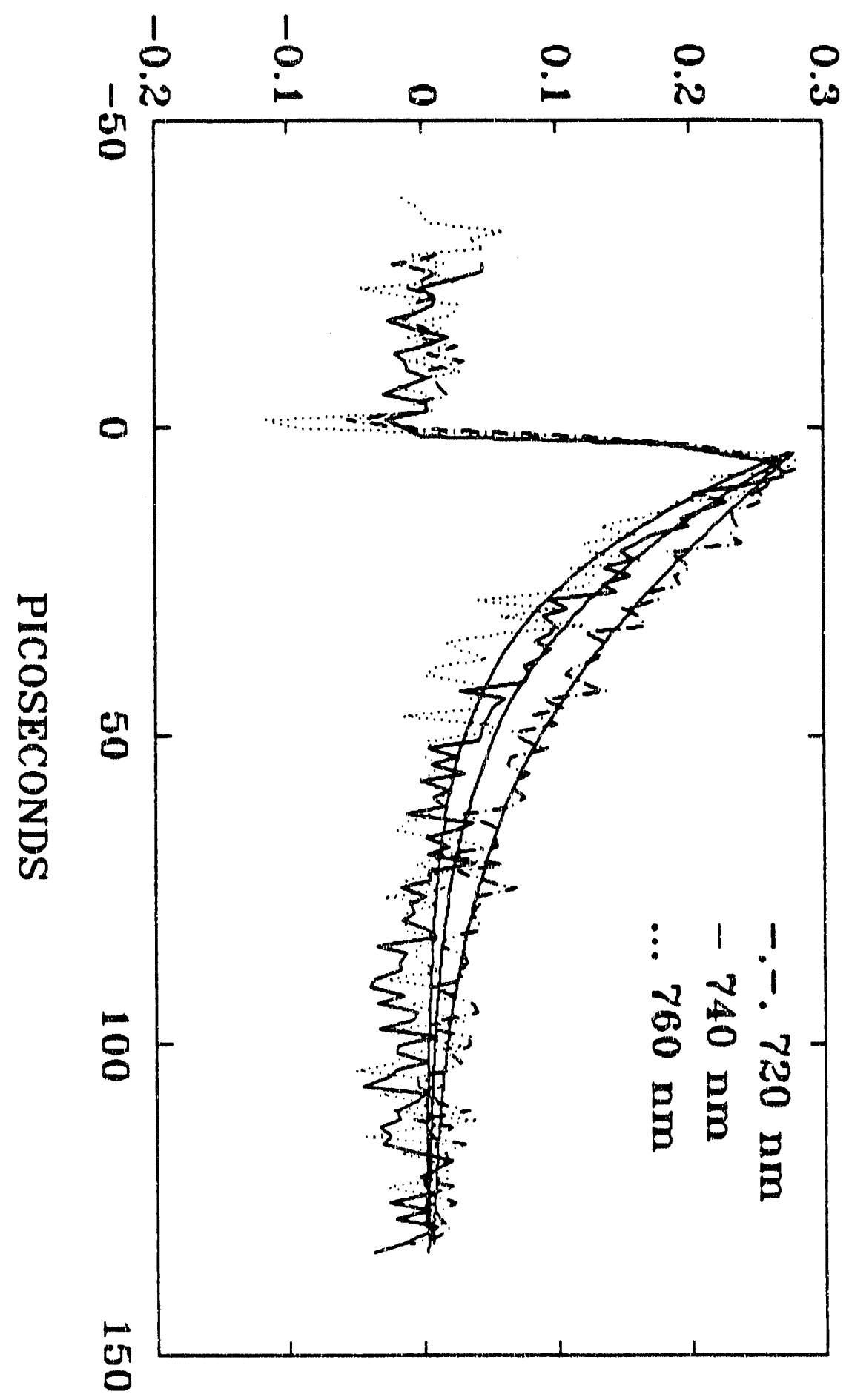

Pigure 4.3 Effect of probe wavelength on cooling times predicted by bulk cooling model and compared to experimental results in $\mathrm{CCl}_{4}$ at $298 \mathrm{~K}$. 
Table 4.3 Thermal conductivities (k) at hot (1100K) and cool ( $T_{\text {eq }}$ - solvent equilibrium temperature) temperatures for solvents in the various thermodynamic states listed.

\begin{tabular}{|c|c|c|c|c|c|}
\hline SOLVENT & $\rho^{*}$ & $\begin{array}{l}T_{\text {eq }} \\
(K)\end{array}$ & $\begin{array}{l}k \text { at } T_{\text {eq }} \\
(W / m K)\end{array}$ & $\begin{array}{l}\mathrm{k} \text { at } 1100 \mathrm{~K} \\
(\mathrm{~W} / \mathrm{m} \mathrm{K})\end{array}$ & $\begin{array}{l}\mathbf{k}_{\text {avg }} \\
(W / m K)\end{array}$ \\
\hline $\mathrm{Xe}$ & 0.57 & 280 & 0.032 & 0.017 & 0.024 \\
\hline $\mathrm{Xe}$ & 0.82 & 280 & 0.057 & 0.023 & 0.040 \\
\hline $\mathrm{Xe}$ & 0.88 & 280 & 0.068 & 0.027 & 0.048 \\
\hline $\mathrm{Xe}$ & 0.95 & 280 & $0.086^{\star}$ & $0.034^{*}$ & 0.060 \\
\hline Xe & 0.88 & 253 & 0.074 & 0.027 & 0.051 \\
\hline $\mathrm{Xe}$ & 0.88 & 298 & 0.064 & 0.027 & 0.046 \\
\hline $\mathrm{Xe}$ & 0.88 & 323 & 0.061 & 0.027 & 0.044 \\
\hline $\mathrm{Kr}$ & 0.57 & 280 & 0.036 & 0.030 & 0.036 \\
\hline $\mathrm{Kr}$ & 0.82 & 280 & $0.068^{\star}$ & $0.044^{*}$ & 0.068 \\
\hline $\mathrm{CH}_{3} \mathrm{OH}$ & 0.73 & 298 & 0.202 & n.a. & n.a. \\
\hline $\mathrm{C}_{6} \mathrm{H}_{14}$ & n.a. & 298 & 0.123 & n.a. & n.a. \\
\hline $\mathrm{CCl}_{4}$ & 1.1 & 298 & 0.104 & n.a. & n.a. \\
\hline $\mathrm{CHCl}_{3}$ & 1.0 & 298 & 0.117 & n.a. & n.a. \\
\hline $\mathrm{CH}_{2} \mathrm{Cl}_{2}$ & 0.96 & 298 & 0.138 & n.a. & n.a. \\
\hline
\end{tabular}

$\rho^{\star}=\rho \sigma^{3}$ where $\rho=$ bulk density, $\sigma=$ molecular radius n.a. = not applicable

* indicates value was interpolated from lower density values.

See Reference 24 for thermal conductivity data. 
Table 4.4 A comparison of experimental cooling times and lifetimes for the internal temperature of the azulene $\left(c_{p} p r^{2} / 3 k\right)$ at various experimental conditions.

\begin{tabular}{|c|c|c|c|c|}
\hline SOLVENT & $\rho^{*}$ & $\begin{array}{l}\mathrm{T}_{\text {eq }} \\
(\mathrm{K})\end{array}$ & $\begin{array}{l}C_{p} \rho r^{2} / 3 k \\
(s e c)\end{array}$ & $\begin{array}{l}\text { Cooling } \\
\text { Time (sec) }\end{array}$ \\
\hline $\mathrm{Xe}$ & 0.57 & 280 & $1.08 \times 10^{-15}$ & $1.70 \times 10^{-10}$ \\
\hline $\mathrm{Xe}$ & 0.82 & 280 & $6.49 \times 10^{-16}$ & $1.75 \times 10^{-10}$ \\
\hline Xe & 0.88 & 280 & $5.45 \times 10^{-16}$ & $1.50 \times 10^{-10}$ \\
\hline Xe & 0.95 & 280 & $4.32 \times 10^{-16}$ & $1.37 \times 10^{-10}$ \\
\hline Xe & 0.88 & 253 & $5.09 \times 10^{-16}$ & $1.50 \times 10^{-10}$ \\
\hline $\mathrm{xe}$ & 0.88 & 298 & $5.68 \times 10^{-16}$ & $1.50 \times 10^{-10}$ \\
\hline $\mathrm{Xe}$ & 0.88 & 323 & $5.88 \times 10^{-16}$ & $1.50 \times 10^{-10}$ \\
\hline $\mathrm{Kr}$ & 0.57 & 280 & $7.17 \times 10^{-16}$ & $1.33 \times 10^{-10}$ \\
\hline $\mathrm{kr}$ & 0.82 & 280 & $3.80 \times 10^{-16}$ & $1.18 \times 10^{-10}$ \\
\hline $\mathrm{CH}_{3} \mathrm{OH}$ & 0.73 & 298 & $1.28 \times 10^{-16}$ & $1.00 \times 10^{-11}$ \\
\hline $\mathrm{C}_{6} \mathrm{H}_{14}$ & n.a. & 298 & $2.1 .0 \times 10^{-16}$ & $1.50 \times 10^{-11}$ \\
\hline $\mathrm{CCl}_{4}$ & 1.1 & 298 & $2.49 \times 10^{-16}$ & $2.20 \times 10^{-11}$ \\
\hline $\mathrm{CHCl}_{3}$ & 1.0 & 298 & $2.21 \times 10^{-16}$ & $2.30 \times 10^{-11}$ \\
\hline $\mathrm{CH}_{2} \mathrm{Cl}_{2}$ & 0.96 & 298 & $1.87 \times 10^{-16}$ & $1.90 \times 10^{-11}$ \\
\hline
\end{tabular}

$\rho^{*}=\rho \sigma^{3}$ where $\rho=$ bulk density, $\sigma=$ molecular radius 
absorlance change as a function of time is fit to the data.

The wavelength dependence has been discussed and shown to fit well with this model. The temperature dependence Figure 4.4 ) is predicted quite accurately by this model. This is partially because the temperature range between the two extreme temperatures is only $70^{\circ}$ which is minor compared to the initial temperature reached by azulere immediately after excitation. Therefore, there is no temperature effect on the cooling of the azulene system.

A remarkable fit is also achieved over the upper range of xenon densities although the fit at low densities is not acceptable (Figure 4.5). The lowest xenon density is near the critical point and in this region thermodynamic values of the system such as thermal conductivity are strongly affected by fluctuations in the solvent approaching supercritical behavior, making the model not useful in that region. Even if this behavior is neglected, it is obvious that the model fails to predict the empirical trend of cooling time with solvent density.

In the case of the krypton density effect (Figure 4.6), the theoretical prediction is not very accurate. It is unfortunate that only two data points are available for this system. The low density condition is not near the critical point for krypton which makes it a reliable value. However, the highest density krypton thermal conductivity value (and the highest density xenon thermal conductivity value as well) 


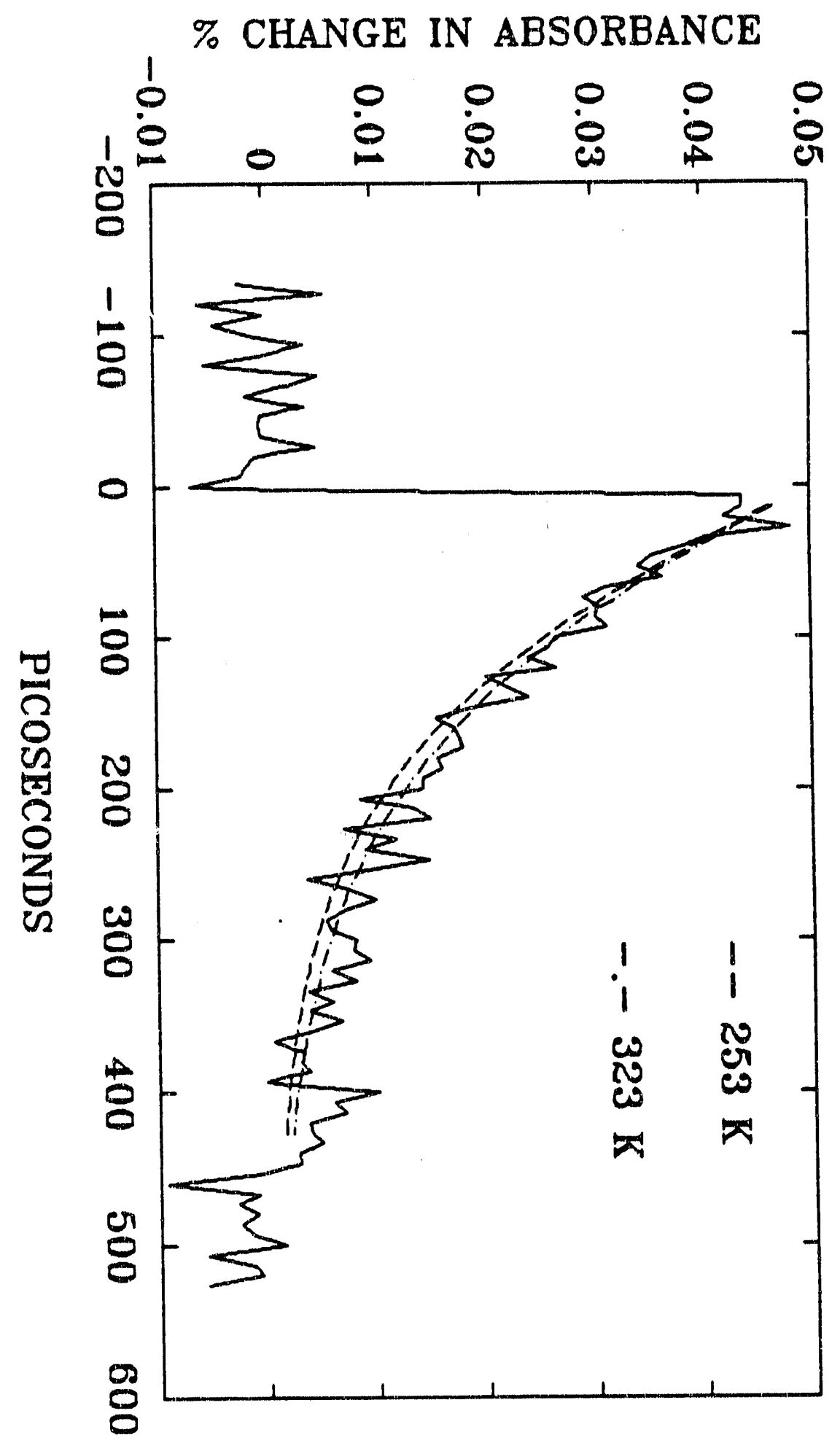

Figure 4. Effect of solvent temperature on cooling time predicted by bulk cooling model and compared to experimental results in liquid xenon at $\rho^{*}=0.88$, probed at $740 \mathrm{~nm}$. 


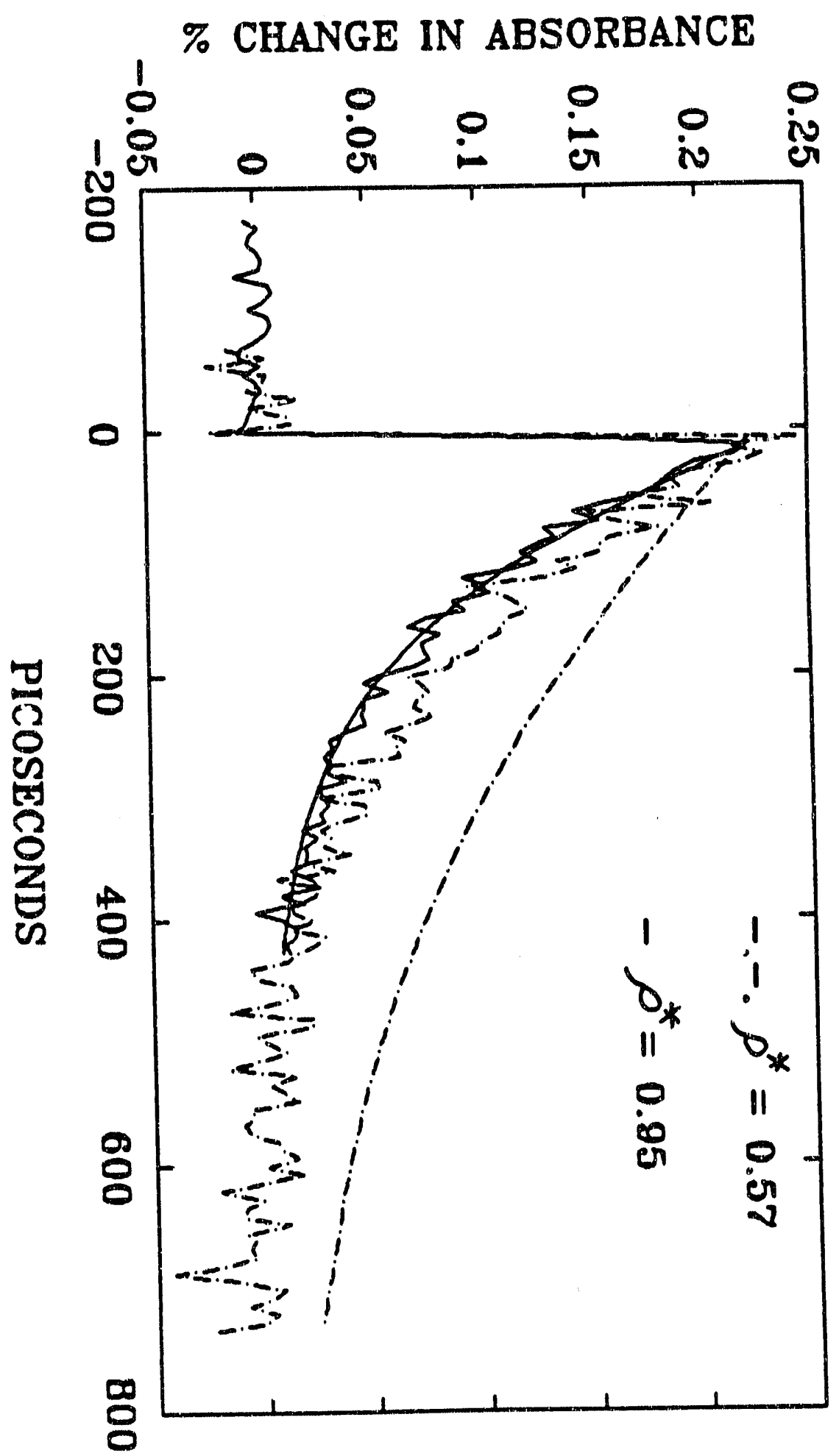

Pigure 4.5 Effect of xenon density on cooling time predicted by bulk cooling model and compared to experimental results at $280 \mathrm{~K}$, probed at $740 \mathrm{~nm}$. 


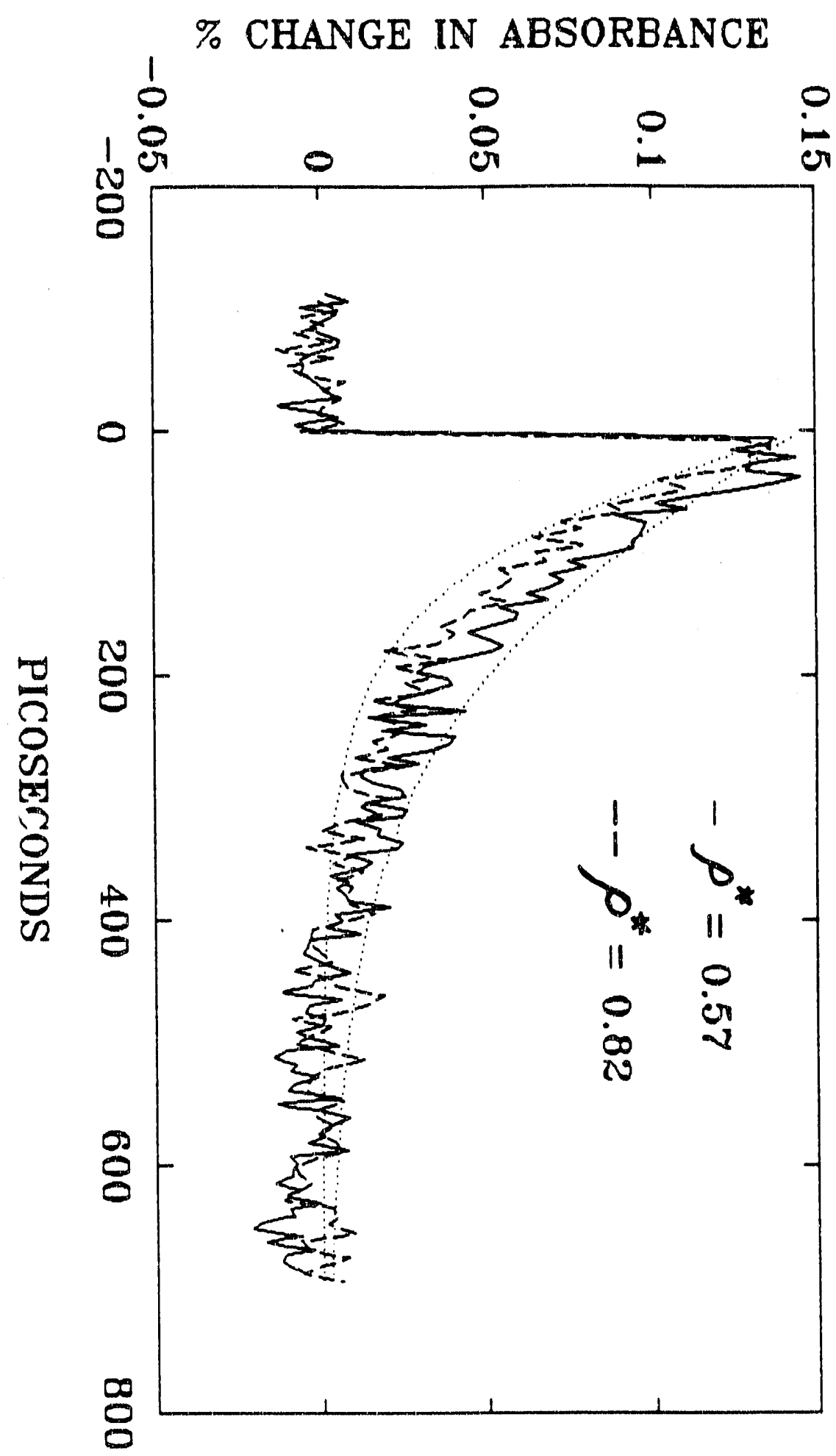

Figure 4.6 Effect of krypton density on vibrational cooling time predicted by bulk cooling model and compared to experimental results at $280 \mathrm{~K}$, probed at $740 \mathrm{~nm}$. 
are interpolated from lowest density values due to the lack of information at those high pressures. This method for obtaining the thermal conductivity may not be sufficiently accurate to predict the behavior in krypton, especially with only two data points.

In spite of the poor fits to the krypton data, the model does predict the difference in time scales between the two noble gas solvents (Figure 4.7). Although the predicted difference is not exact, probably due to inaccuracies in the krypton value, the general rate change seen between krypton and xenon can be attributed to their difference in thermal conductivity.

When used to compare results obtained in conventional solvents, such as hexane, methanol, and the chloromethanes, the model irregularly follows the experimental results. Thermal conductivities at $1100 \mathrm{~K}$ could not be obtained so the equilibrium temperature thermal conductivities were used. The chloromethanes have similar thermal conductivities at room temperature and the vibrational cooling rate in these solvents is also very similar. Methanol has a large thermal conductivity which would lead one to believe that it should decay quicker than the other solvents, and it does. Hexane has a thermal conductivity similar to the chloromethanes and yet the vibrational cooling rate is much more rapid. Something else may be affecting the process of vibrational cooling in hexane, possibly resonance between vibrational 


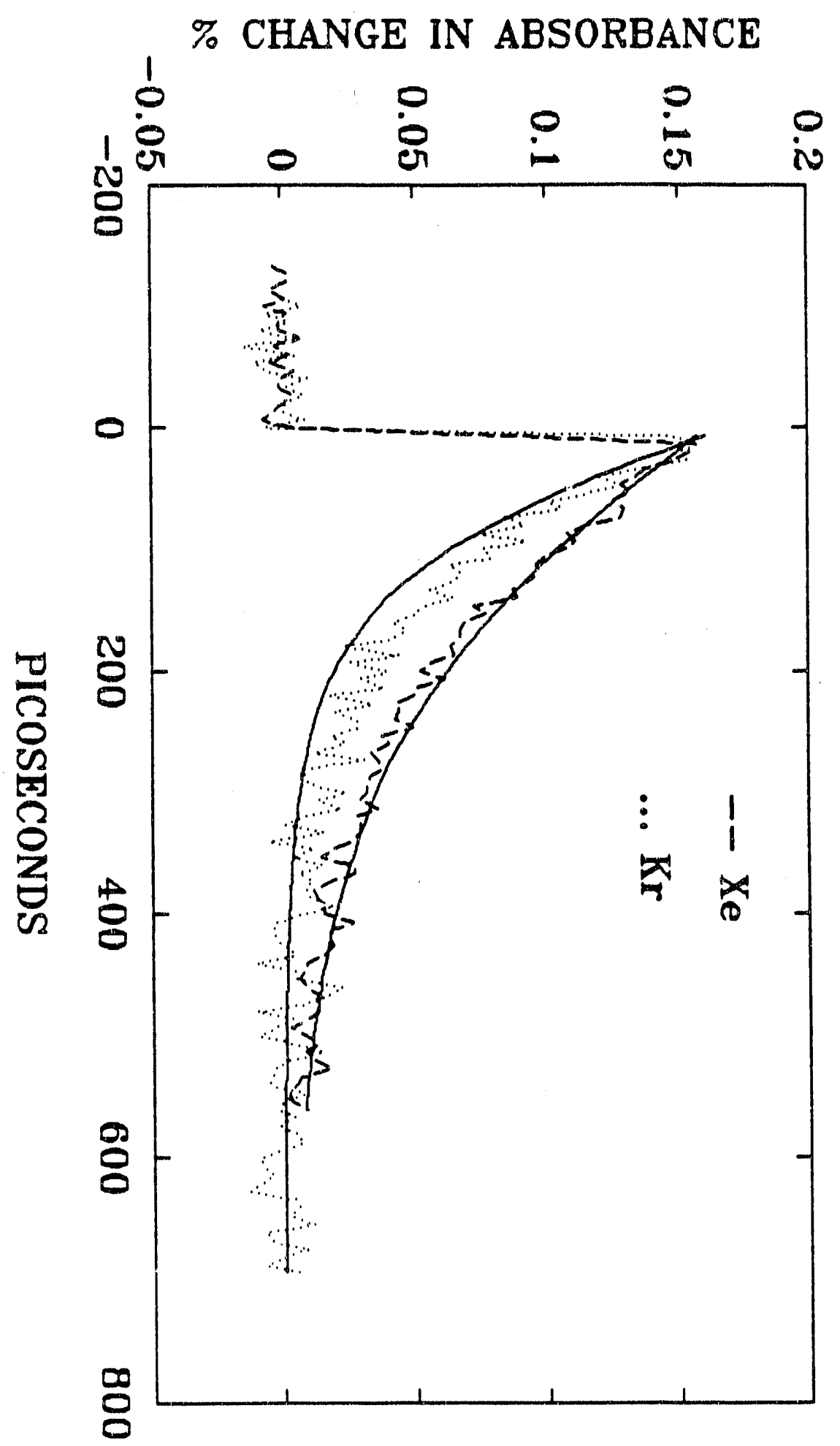

Pigure 4.7 Effect of solvent (xenon vs. krypton) on cooling time predicted by bulk cooling model and compared to experimental results at $280 \mathrm{~K}, \rho^{*}=0.82$. 
modes in the two hydrocarbons, azulene and hexane. Thermal conductivity may be only part of the explanation for the change in vibrational cooling rates.

It is interesting that solvent thermal conductivities parallel some of the empirical trends in vibrational cooling lifetimes. This model not only incorporates physical parameters of the solvent but also incorporates the temperature of the solvent and the probe wavelength. These different espects of the experiment will affect the end result. This model is useful because it accurately predicts so many of the different trends seen empiricalily. However, there are problems with this method. First and foremost, it does not predict exact exponential relaxation, since the exponential temperature term is not the only term which is used to fit the decay dynamics. Exponential relaxation is seen empixically and has been predicted theoretically. ${ }^{22}$ Essentially, the form is very similar and small nonexponential differences would be hard to distinguish because of the noise of the data. However, in some cases, the nonexponential shape reduces the quality of the predicted fit. In addition, this model does not illuminate the microscopic picture of azulene vibrational relaxation. That thermal conductivity is important to the cooling process coes not explain what specific methods of energy transter are occurring between solute and solvent; what modes are involved and how collisions are affected. Perhaps this model does 
indicate that when a number of vibrational modes in a molecule are excited, the large scale effect of all of them requires a different description of the system and that this description approaches the larger description of bulk thermal transport in Iiquids.

The results of this calculation do not exactly predict a density dependence in the vibrational cooling time. It is understandable that this model, which incorporates bulk thermal conductivities to explain iolecular vibrational energy transfer, might not work to describe the effect of solvent density on energy transfer. on a microscopic level, the magnitude of thermal conductivity in a liquid is simply related to the collision frequency between solvent molecules. Therefore the magnitude of the thermal conductivity should scale as the collision frequency with density, and it does approximately. Thexefore thermal conductivity cannot possibly do a better job than IBC in explaining the data. Bulk parameters cannot be directly and simply applied to explain the molecular level dynamics of azulene.

\subsection{Liquid Phase Vibrational Relaxation studies of Azulene}

The liquid phase results consist of two studies, the first ${ }^{18}$ providing basic results on ground state azulene in solution and the second ${ }^{25}$ giving more detailed information. The studies are similar to this current study in that a visible picosecond pump-probe system was used to monitor the 
azulene. A pump wavelength of $527 \mathrm{~nm}\left(19,000 \mathrm{~cm}^{-1}\right)$ was used instead of $590 \mathrm{~nm}\left(17,000 \mathrm{~cm}^{-1}\right)$ and the laser pulse is somewhat longer, $5 \mathrm{psec}$, reducing the time resolution of their results. A comparison of the results of the two studies is shown in Table 4.5 .

The theory put forth to try to explain these trends seen in the data tries to incorporate information describing the energy flow through the solvent, i.e. the solvent diffusivity. This idea is very similar to the bulk thermal model described earlier. Including this solvent parameter into the calculation allows them to examine the flow of energy from the hot molecule into the surrounding solvent. The loss of energy from the hot azulene to the first solvent shell is described by IBI theories. The cooling of the hot solvent shell is then described by macroscopic heat flow equations such a Fick's Law. The size of the solvent shell is adjusted in order to fit the data. It appears that this works well in describing their results as they obtain reasonable solvent shell sizes for all the different solvents used.

A table of the diffusivities of the noble gas solvents is given in Table $4.6 .^{26}$ These values are entirely within the range of diffusivities for conventional solvents. However, there is an incredible difference in the structure and complexity of these noble gas solvents and the molecular solvents used by sukowski et al. ${ }^{25}$ As a result the noble gas solvents cannot dissipate a great deal of energy in a single 
Table 4.5 Comparison of current results and Kaiser's results

\begin{tabular}{|l|l|l|l|l|}
\hline & \multicolumn{2}{|l|}{$\begin{array}{l}\text { Results - Ref. 25 } \\
\text { Pump at 527 nm }\end{array}$} & \multicolumn{2}{|l|}{$\begin{array}{l}\text { Current Results } \\
\text { Pump at 590 nm }\end{array}$} \\
\hline SOLVENT & $\begin{array}{l}\text { COOLING } \\
\text { TIME } \\
\text { (psec) }\end{array}$ & $\begin{array}{l}\text { WAVELENGTH } \\
\text { (nm) }\end{array}$ & $\begin{array}{l}\text { COOLING } \\
\text { TIME } \\
\text { (psec) }\end{array}$ & $\begin{array}{l}\text { WAVELENGTH } \\
(\mathrm{nm})\end{array}$ \\
\hline $\mathrm{CCl}_{4}$ & $40 \pm 15$ & 735 & $22 \pm 4$ & 740 \\
\hline $\mathrm{CHCl}_{3}$ & $35 \pm 10$ & 725 & $23 \pm 3$ & 740 \\
\hline $\mathrm{CH}_{3} \mathrm{OH}$ & $13 \pm 5$ & 730 & $10 \pm 3$ & 740 \\
\hline
\end{tabular}


Table 4.6 Noble gas diffusivities $\left(k=(M W \cdot k) /\left(p \cdot C_{p}\right)\right)$ where $k$ is thermal conductivity, $C_{p}$ is heat capacity, $M W$ is molecular weight and $p$ is bulk density.

\begin{tabular}{|l|l|l|l|l|}
\hline SOLVENT & $\begin{array}{l}\rho \\
(\mathrm{g} / \mathrm{mI})\end{array}$ & $\begin{array}{l}\mathrm{k} \\
(\mathrm{W} / \mathrm{mK})\end{array}$ & $\begin{array}{l}\mathrm{C}_{\mathrm{p}} \\
(\mathrm{J} / \mathrm{moleK})\end{array}$ & $\begin{array}{l}\text { DIFFUSIVITX } \\
\left(\mathrm{cm}^{2} / \mathrm{sec}\right)\end{array}$ \\
\hline $\mathrm{Xe}$ & 1.8 & $0.032^{+}$ & $70^{+}$ & $3.3 \times 10^{-4}$ \\
\hline $\mathrm{Xe}$ & 2.6 & 0.057 & 40.1 & $7.2 \times 10^{-4}$ \\
\hline $\mathrm{Xe}$ & 2.8 & 0.068 & 37.8 & $8.4 \times 10^{-4}$ \\
\hline $\mathrm{Xe}$ & 3.0 & $0.086^{\star}$ & $36-38^{\star}$ & $1.0 \times 10^{-4}$ \\
\hline $\mathrm{Kr}$ & 1.6 & 0.036 & 41.0 & $5.4 \times 10^{-4}$ \\
\hline $\mathrm{Kr}$ & 2.3 & $0.068^{\star}$ & $33^{*}$ & $1.0 \times 10^{-3}$ \\
\hline
\end{tabular}

+ This thermodynamic state is very near the critical point, causing the value of the heat capacity and thermal conductivity to vary a great deal over a small temperature and pressure range.

* These values have been interpolated from lower density data.

See Reference 24 for thermal conductivity data.

See Reference 26 for heat capacity data. 
collision with a hot azulene molecule as can molecular solvents. This would indicate that differences in diffusivity alone will not sufficiently compensate for the differences in the dynamics in the two solvents. This would seem to indicate that this model cannot accurately predict the minimal temperature change seen in the data. Based on the previous analysis done on the current azulene results, it is doubtful that a theory incorporating the diffusivity will do a better job predicting the relaxation dynamics in noble gas solvents than the theories described earlier do.

Unfortunately, the magnitude of the calculations required to make this theory work, nake it impossible to apply to this current data. It seems that their theory works very well in fitting their data and provides some physical insight into the process of relaxation. It is quite interesting that their theory incorporates various aspects of the theories used in this current work. It would be very interesting to apply their theory to this current data to see if the cooling of a hot solvent shell described by solvent diffusivity accurately describes the relaxation dynamics of azulene in these unique noble gas liquids. 


\section{References for Chapter 4}

1. Mark E. Paige, Ph.D. thesis, Density effects on the Molecular Dynamics of the $I_{2}$ Geminate Recombination Reaction in Liquid Xe, University of California - Berkeley, Berkeley, CA 1990 .

2. M.E. Paige and C.B. Harris, Chem. Phys. 149, 37 (1990).

3. M.E. Paige and C.B. Harris, J. Chem. Phys. 93, 3712 (1990).

4. R.M. Hochstrasser and C.A. Nyi, J. Chem. Phys. 70, 1112 (1979) •

5. R.N. Schwartz, Z.I. Slawsky, and K.F. Herzfeld, J. Chem. Phys. 20, 1591 (1952).

6. T. Einwohner and B.J. Alder, J. Chem. Phys. 49, 1458 (1968); Paul K. Davis and Irwin Oppenheim, J. Chem. Phys. 57, $505(1972)$.

7. H. Hippler, B. Otto, and J. Troe, Ber. Bunsenges. Phys. Chem. 93, 428 (1989).

8. Michel J. Rossi, Jack R. Pladziewicz, and John R. Barker, J. Chem. Phys. 78, 6695 (1983).

9. Donald A. McQuarrie, Statistical Mechanics, (Harper \& Row, New York, 1976), pp. 259.

10. Daniel J. Russell, Ph.D. thesis, Vibrational Relaxation in Liquids: Comparisons between Gas Phase and Liquid Phase Theories, University of California - Berkeley, Berkele, CA 1990 .

11. Samuel Leutwyler and Juerg Boesiger, z. Phys. Chem. Neue Folge 154, 31 (1987).

12. Karen E. Schultz, Daniel J. Russell, and Charles B. Harris, submitied to J. Chem. Phys.

13. J.K. Brown, C.B. Harris, and John C. Tully, J. Chem. Phys. 89, 6687 (1.988).

14. Kieran F. Lim and Robert G. Gilbert, J. Phys. Chem. 94, 77 (1990).

15. David Chandler, Acc. Chem. Res. 7, 246 (1974). 
16. N.H. Gottfried, A. Seilmeier and W. Kaiser, Chem. Phys. Lett. 111, 326 (1984).

17. A. Seilmeier, P.O.J. Scherer and W. Kaiser, Chem. Phys. Lett. 105,140 (1984).

18. W. Wild, A. Seilmeier, N.H. Gottfried, and W. Kaiser, Chem. Phys. Lett. 119, 259 (1985).

19. R.J. Sension, S.T. Repinac, and R.M. Hochstrasser, J. Chem. Phys. 93, 9185 (1990).

20. Frank Kreith, Principles of Heat Transfer, 3 rd ed. (Intext Educational Publishers, New York, 1973), pp. 141, 401; C.O. Bennett and J.E. Myers, Momentum, Heat and Mass Transfer, 3 rd ed. (McGraw-Hill, New York, 1982), pp. 310-311.

21. Aviv Amirav and Joshua Jortner, J. Chem. Phys. 81, 4200 (1984).

22. Wendel1 Forst, Chem. Phys. 147, 241 (1990).

23. Carl L. Yaws, H.M. Ni, and P.Y. Chiang, Chemical Engineering 95:7, 91 (1988).

24. Thermal conductivities for xenon and krypton at various densities were obtained from the following references: H.J.M. Hanley, R.D. McCarty, and W.M. Haynes, J. Phys. Chem. Ref. Data 3, 979 (1974); V.A. Rabinovich, A.A. Vasserman, V.I. Nedostrup, and L.S. Veksler, Thermophysical Properties of Neon, Argon, Krypton, and Xenon, (Hemisphere Publishing Corp., Washington, 1976), pp. 501-506, 584-588. Thermal conductivity of $\mathrm{CH}_{2} \mathrm{H}_{2}$ from N.V. TSederberg, Thermal conductivity of Gases and Liquids, (Cambridge, MIT press, 1965), pp. 199. Thermal conductivities for all other solvents were obtained from Y.S. Touloukian, Thermophysical properties of Matter vol.3, (Plenum, New York, 1970), pp. 156, 161, 214, 223.

25. U. Sukowski, A. Seilmeier, T. Elsaesser, and S.F. Fischer, J. Chem. Phys. 93, 4094 (1990).

26. Heat capacities for xenon and krypton at various densities were obtained from: V.A. Rabinovich, A.A. Vasserman, V.I. Nedostrup, and I.S. Veksler, Thermophysical Properties of Neon, Argon, Krypton, and Xenon, (Hemisphere Publishing Corp., Washington, 2976), pp. 577-558, 494-495; J.A. Cowan and J.W. Leech, Can. J. Phys. 59, 1280 (1981); Jan Jůza and Oldrich Sifner, Acta. Technica ČSAV 1, 1 (1976). 


\section{Chaptex 5: SUMMARY AND CONCLUSIONS}

Before these current experiments on azulene in compressed noble gases were begun, little was known of the role solvent plays in viurationally relaxing azulene and its effect on the time scale of the dynamics. previous experiments on this system provide some information on the vibrational relaxation process. Spectroscopic studies show that skeletal modes (such as the bridging bond stretch mode) in azulene in the range $1200 \mathrm{~cm}^{-1}$ to $1700 \mathrm{~cm}^{-1}$ are activated by the internal conversion process and vibrational relaxation. However, the low frequency modes in the molecule would have the largest coupling with the low frequency solvent modes and are therefore more likely to act as the energy drain from the molecule to the solvent. The wavelength dependence of the vibrational cooling time probes this frequency range of hot bands and supports the fact that high frequency modes decay into low frequency modes, yielding longer cooling times for low frequency modes. The high frequency $\mathrm{C}-\mathrm{H}$ stretches are not involved in the relaxation dynamics of azulene, as evidenced by the absence of a solvent isotope effect.

other aspects of solvent mediation of the relaxation process were studied, including solvent density, temperature and solvent mass. The relaxation rate was faster in krypton solvents compared to xenon solvents at the same reduced densities. The solvent density (over the range $\rho^{\star}=0.57$ - 
0.95 ) and solvent temperature (over the range $\mathrm{T}=253 \mathrm{~K}-323 \mathrm{~K}$ ) had a minimal effect on the relaxation rate. Although a bottleneck in the energy flow has been seen in other studies of vibrational relaxation, it is not believed to occur in this system.

There are a number of ways to model these empirical results. Using a bulk solvent parameter such as thermal conductivity to describe the solvent continuum accurately predicts the temperature independence, but works only moderately in predicting the mass effect and predicts a large solvent density effect where there is none empiricaliy. Apparently thermal conductivity does not sufficiently account for the microscopic interactions between solute and solvent which affect the vibrational cooling process.

Isolated Binary collision theory provides an alternative description of the dynamics of azulene. IBC theory, in conjunction with gas phase energy transfer studies, can be easily applied to azulene in noble gas solvents. The gas phase studies show that the probability of energy transfer is similar in xenon and krypton and also is similar at high temperature and low temperatures. Therefore, the difference in relaxation rates under different experimental conditions is solely due to differences in collision frequency.

simple collision frequency calculations indicate that the collision frequency does change as a function of solvent parameters such as temperature, density and solvent mass. In 
particulax, the Davis-oppenheim model for condensed phase collision frequencies quite accurately predicts the minimal temperature dependence. Similarly, the change in relaxation time with solvent mass is roughly predicted by this model; the ratio of experimental cooling times in the xenon and krypton solution is approximately equal to the $\left(\mu_{\mathrm{AzKr}} / \mu_{\mathrm{AzXe}}\right)^{1 / 2}$, as predicted by theory. Molecular dynamics simulations support the assumption that the difference in the dynamics in xenon and krypton is largely because of the difference in the mass of the two solvents, and not because of differences in the potentials.

The anomalous independence of the vibrational cooling rate and solvent density is surprising in that it contradicts what one might guess would occur in a liquid. Increasing solvent density causes molecules to be more tightly packed, increasing the number of collisions and solute-solvent interactions. To first order, these processes should affect vibrational relaxation which is dependent vpon solute-solvent interactions. This is not the case for azulene in compressed noble gases and is not predicted by current IBC theories. IBC theories predict a change in relaxation rates much larger than the $20 \%$ change seen over the experimental density range.

To use the Davis-oppenheim model to calculate collision frequencies as a function of density, one must only consider how the term $\rho g\left(R^{*}\right)$ changes as a function of density since all other terms cancel out if two experimental conditions are 
ratioed. If one assumes a spherical Lennard-Jones interaction between the azulene and the solvent, the Davis-oppenheim model predicts a density effect on the order of the 3008 change seen in the iodine experiments and simulations. This indicates how poorly the implicit assumptions in the Davis-oppenheim model of a spherical $g\left(R^{*}\right)$ describe the azulene molecule.

It is not surprising that azulene in solution cannot accurately be described by a sphere. It is a complicated, anisotropic molecule. Its aromaticity could easily give it different solvent interactions perpendicular and parallel to the ring plane. In fact, gas phase cluster experiments show that aromatic-noble gas clusters often have the noble $9:$ molecule packed directly above and below the rings. It is obvious that if IBC theories are to be used to describe the dynamics of azulene in noble gas liquids, the spherical assumptions must be eliminated.

one way to obtain three dimensional structural information about the solvent is by using molecular dynamics simulations. Given the interaction potentials of the solvent and the solute, these simulations calculate the cquilibrium packing geometry of an asymmetric molecule like azulene surrounding by xenon atoms.

Preliminary results indicate that there is an anisotropic distribution of solvent molecules around the azulene. The favorable packing configurations have xenon atoms above and below the azulene ring system, although their exact placement 
relative to the rings is not yet known. Because of the relative size of azulene and xenon, it is possible that one or two xenon atoms may sit above and below the two carbon rings in azulene.

If these types of solvent arrangement occur in noble gas liquids, the packing may prohibit the approach of other solvent atoms, thus inhibiting the cooling process regardless of solvent density. Preliminary simulation results indicate that the packing above and below the azulene ring does not change as a function of density. If the low frequency out-ofplane bending modes are important in azulene relaxation but are being blocked from collisions with the solvent by quasibound solvent atoms above and below the azulene, a density independent relaxation rate might be possible. The three dimensional complexity of this solute atom uniquely affects the influence of the solvent on the energy transfer dynamics. When a three dimensional picture of the solvent structure around the azulene is available, the anisotropic radial distribution function can be calculated and put into the IBC equations. Hopefully, this adaptation of current IBC theories will facilitate applications of these models to more realistic and complex molecular systems. 


\section{APPENDIX}

The following is the program used to determine the internal temperature of the azulene given the excitation energy put into the molecule. Relevant literature references for the backbone of the code as well as the molecular parameters used in the calculation are cited in comments within the program.

Basically, this program uses a given set of vibrational frequencies for a molecule and, assuming a Boltzmann distribution of population in the modes, calculates the internal energy using the following equation:

$$
\langle E\rangle=\frac{\int E \rho(E) \exp \left(-E / k_{B} T\right) d E}{\int \rho(E) \exp \left(-E / k_{B} T\right) d E}
$$

where $\rho(E)$ is the density of states. This calculation relates average internal energy, $\langle E\rangle$, and a corresponding internal temperature, $T$. It should be noted that rotational information is not incorporated into this calculation.

Generally, the energy is integrated to infinity. However, to use the program a finite upper limit on the average energy must be set to facilitate calculation. The upper energy limit should not affect the value of the average energy returned. For example, an upper limit of $35,000 \mathrm{~cm}^{-1}$ or more was sufficient to yield consistent values of $T$ and $\langle E\rangle$ for the azulene molecule, i.e. $\langle E\rangle$ is $17,000 \mathrm{~cm}^{-1}$, the excitation energy, and $T$ is $1100 \mathrm{~K}$, the internal molecular 
temperature corresponding to that amount of energy. A limit lower than $35,000 \mathrm{~cm}^{-1}$ did not yield these values. In calculating the room temperature internal energy, an upper limit of $15,000 \mathrm{~cm}^{-1}$ was used. The program calculated an internal energy of $1100 \mathrm{~cm}^{-1}$ for azulene at $300 \mathrm{~K}$.

To use the program to calculate the internal temperature for azulene, little needs to be altered. The vibrational mode frequencies and the number of modes shown in the program below are correct for azulene. However, this program can and has been used successfully to calculate tine internal energy of many different polyatomic molecules. For another molecule, the mode parameters mentioned above must be changed in order to incorporate the detailed vibrational structure of the molecule under investigation. 
/ This is the program for calculating the unimolecular */

/* rate constants by $\mathrm{RRKM}$ theory * /

$/ * \mathrm{k}(E)=\mathrm{W}(\mathrm{E}-\mathrm{EO}) / \mathrm{rho}(\mathrm{E})$ */

/* This program will calculate the number of states at $E * /$

$/ *$ and total number $* /$

/* of states by Beyer-Swinehart algorithm and store */

* data on disik for later use */

/ The algorithm for reac() can be found in a paper by */

/ * Stein \& Rabinovitch */

/* in the Journal of Chemical Physics Vol. 58, \#6, */

* p. 2438 (1973) */

/* This program was obtained from Dr. Minyung Lee with */

$/$ * permission of the creator. */

\#include <stdio.h>

\#include <math. $h>$

\#define $D 48 / * D$ is the number of modes in the molecule */ \#define $\operatorname{MAX} 35000 / * \operatorname{MAX}$ is the upper energy limit in $\mathrm{Cm}-1 * /$ \#define $\mathrm{EB} 1259 / \mathrm{EB}$ is the barrier height in $\mathrm{cm}-1$ */

float $\mathrm{ph}=3.337 \mathrm{e}-11 ; \quad / *$ Plank constant in $\mathrm{cm}-1$ *

float boltz $=0.6993 ; \quad / *$ boltzmann constant in $\mathrm{cm}-1$ */

long float den[MAX], sum[MAX];

float ke[MAX], scf,cfc, $q$, qn;

int $\mathrm{d}[\mathrm{D}]=\{3091,3074,3059,3024,3002,1638,1580,1443$, $1392,1294,1117,1054,899,671,559,492,403,1000,908$, $795,531,323,163,1000,960,946,764,721,664,492,311$, $165,3044,3007,1694,1580,1479,1453,1301,1205,1151$, $1007,970,721,593,559,478,3083\}$;

/ $\mathrm{d}$ is array containing the modes of the molecule in $\mathrm{cm}-1$ */ /* The values of $d$ for azulene were obtained from a paper */

* by 0 . Gebhardt */

/* in the journal ACTA Chemica scandinavica Vol.27, */

$/ * \# 5$, pp. 1725 (1973). */

$\operatorname{main}()$

l

float koft, fkt(), avet, vpf():

printf("\nenter the scale factor: "):

scanf ("qe", \&scf);

printf("\nEnter the centrifugal correction: ");

scanf ("qe", \&cfc);

reac(): / * calculate density of state and save. $1 \mathrm{~cm}-1$ grain */ comp ()$; \quad / *$ calculate level sum $* /$

ka(): $\quad /$ calculate RRKM rate $* /$

vpf(300.0); $\quad /$ * calculate density of state integrals */

avet $=q n / q ; \quad /$ average internal energy is quotient * $/$

$1 *$ of 2 incegrals over energy */

koft $=f k t(300.0) ; \quad / * k(t)$ is the $R R K M$ rate */

printf ("\naverage energy $=$ ze $k(t)=$ se", avet, koft); 


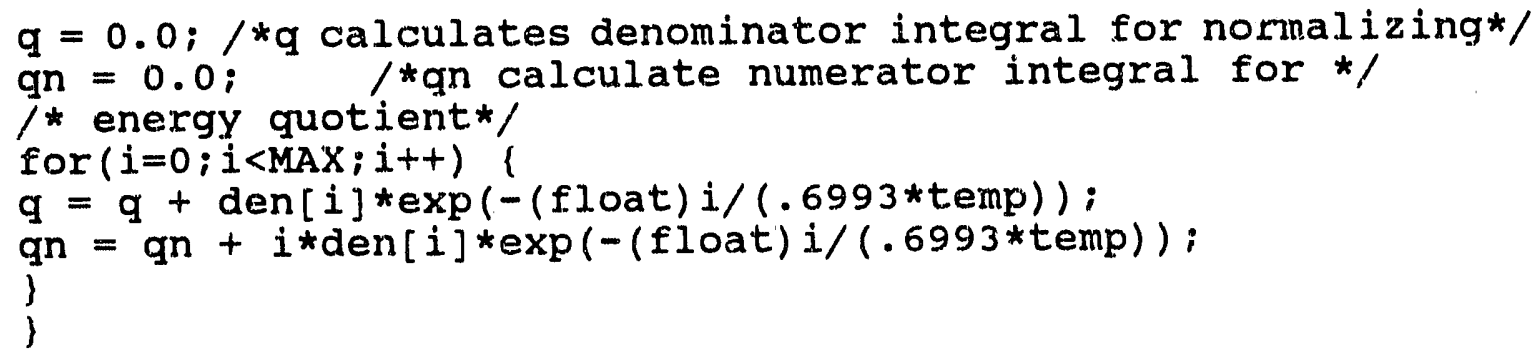



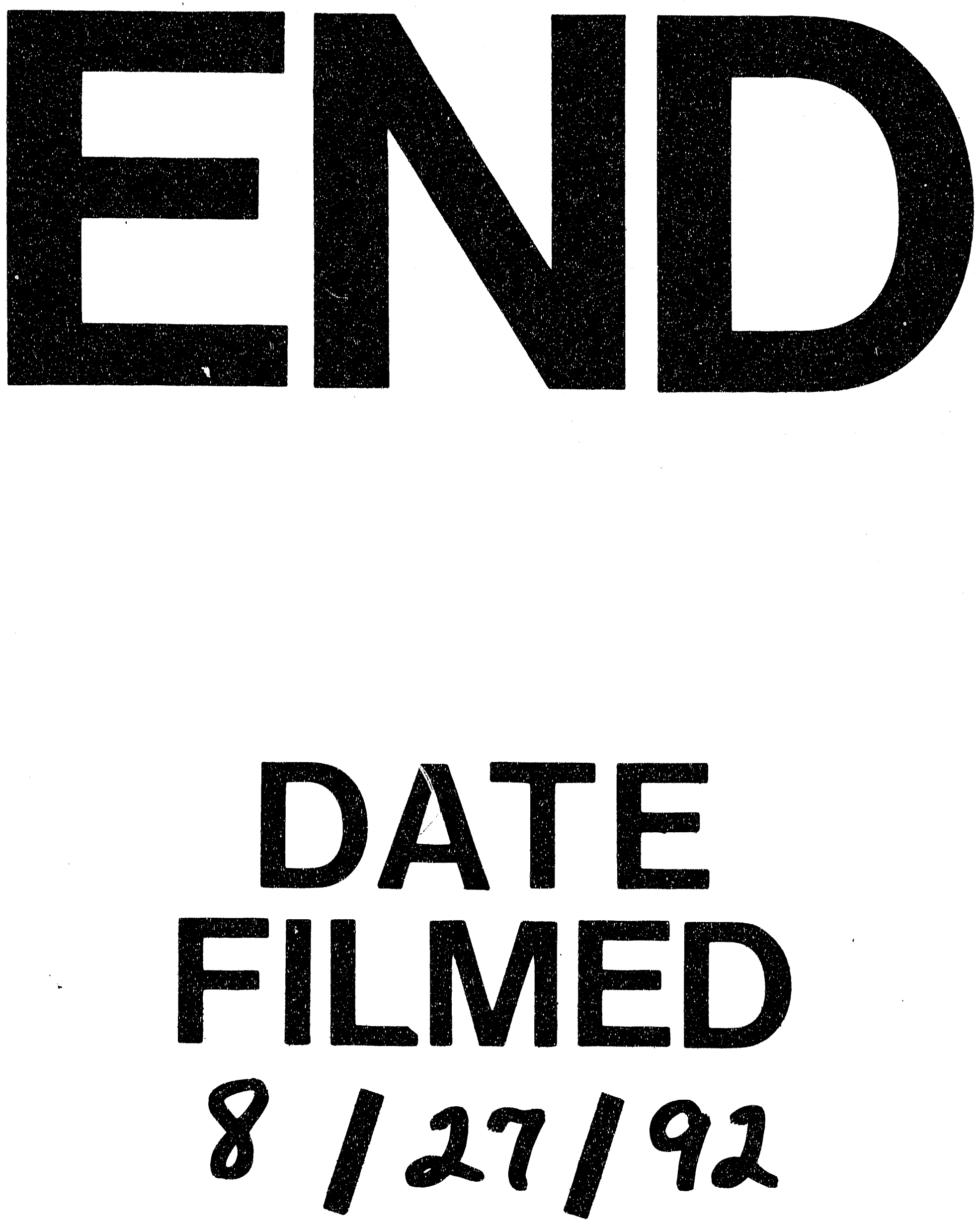

I 
